\title{
Saponins from Saffron Corms Inhibit the Gene Expression and Secretion of Pro- Inflammatory Cytokines
}

Morris Keller ${ }^{1}$, Sarah Fankhauser ${ }^{2}$, Noreen Giezendanner ${ }^{2}$, Michelle König ${ }^{2}$, Franziska Keresztes ${ }^{1}$, Ombeline Danton ${ }^{1}$, Orlando Fertig ${ }^{1}$, Laurence Marcourt ${ }^{3}$, Matthias Hamburger $^{1,{ }^{*} \text {, Veronika Butterweck }}{ }^{2,}{ }^{*}$, Olivier Potterat ${ }^{1}$

\section{"Corresponding Authors}

Veronika Butterweck - School of Life Sciences, University of Applied Sciences, Northwestern Switzerland, Muttenz, Switzerland; Current address: Max Zeller Söhne AG, 8590 Romanshorn, Switzerland; Phone: +41-71-0466-447; Email:

Veronika.Butterweck@ZellerAG.ch; orcid.org/0000-0001-7516-8789

Matthias Hamburger - Pharmaceutical Biology, Pharmacenter, University of Basel, 4056 Basel, Switzerland; Phone: +41-61-207-1425; Email: matthias.hamburger@unibas.ch; orcid.org/0000-0001-9331-273X

\footnotetext{
${ }^{1}$ Pharmaceutical Biology, Pharmacenter, University of Basel, 4056 Basel, Switzerland

${ }^{2}$ School of Life Sciences, University of Applied Sciences, Northwestern Switzerland, Muttenz, Switzerland
}

${ }^{3}$ School of Pharmaceutical Sciences and Institute of Pharmaceutical Sciences of Western Switzerland (ISPSW), University of Geneva, CMU, 1211 Geneva 4, Switzerland 
Figure S 1. Effect of the compounds 1-10 on cell viability of HaCaT cells compared to the control (DMEM incl. $0.1 \%$ DMSO). The results represent three independent experiments $(n=3)$ and are presented as mean \pm standard deviation. ${ }^{*} p \leq 0.05$ vs. control, ${ }^{* *} p \leq 0.01$ vs. control, $* * * p \leq 0.001$ vs. control...... 4

Figure S2. Isolation scheme for the isolation of substances I-VI. ....................................................... 4

Figure S3. Isolation scheme for the isolation of compounds 1-10.................................................. 5

Table S1. ${ }^{1} \mathrm{H}$ and ${ }^{13} \mathrm{C}$ NMR Spectroscopic Data of the Aglycone and Fatty Acid Residue in Compounds 12 (DMSO-d 6 ; $500 \mathrm{MHz}$ for ${ }^{1} \mathrm{H}$ and $126 \mathrm{MHz}$ for ${ }^{13} \mathrm{C} \mathrm{NMR} ; \delta$ in ppm)

Table S2. ${ }^{1} \mathrm{H}$ and ${ }^{13} \mathrm{C}$ NMR Spectroscopic Data of the Glycosidic Moiety in Compounds 1-2 (DMSO- $\mathrm{d}_{6}$; $500 \mathrm{MHz}$ for ${ }^{1} \mathrm{H}$ and $126 \mathrm{MHz}$ for ${ }^{13} \mathrm{C} \mathrm{NMR} ; \delta$ in ppm).

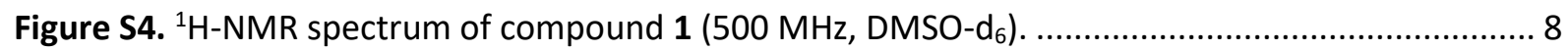

Figure $55 .{ }^{13} \mathrm{C}$-DEPTq spectrum of compound 1 (126 MHz, DMSO-d $\left.\mathrm{d}_{6}\right)$............................................. 8

Figure S6. ${ }^{1} \mathrm{H}-{ }^{1} \mathrm{H}$ COSY spectrum of compound 1 (500 MHz, DMSO- $\left.\mathrm{d}_{6}\right)$........................................ 9

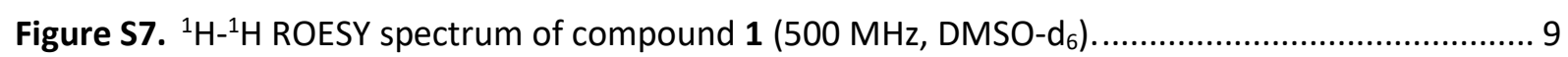

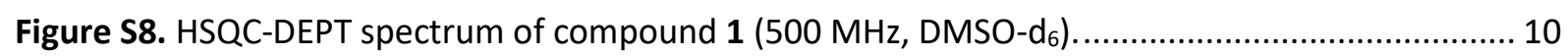

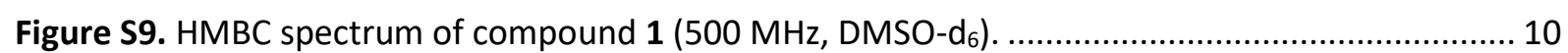

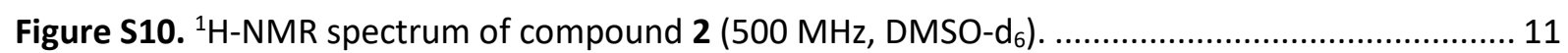

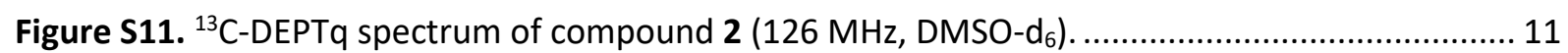

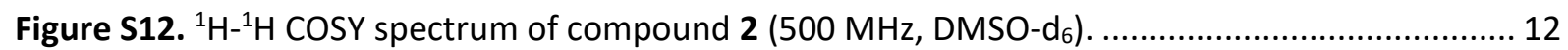

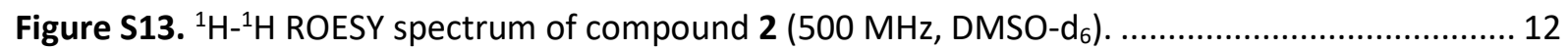

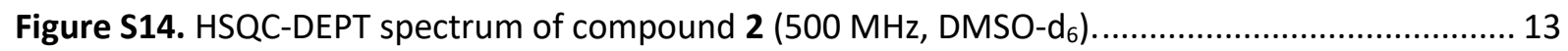

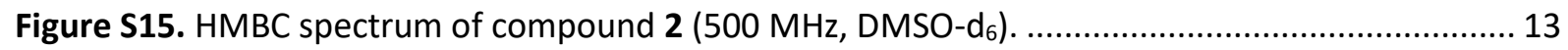

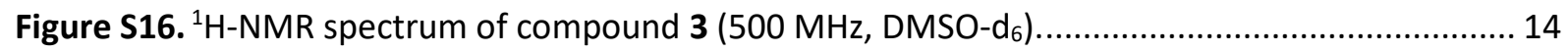

Figure S17. ${ }^{13} \mathrm{C}$-DEPTq spectrum of compound $3\left(126 \mathrm{MHz}\right.$, DMSO-d $\left.\mathrm{d}_{6}\right)$........................................ 14

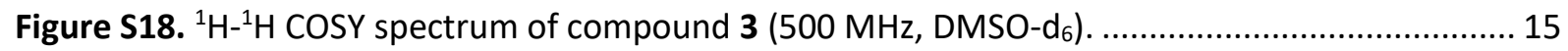

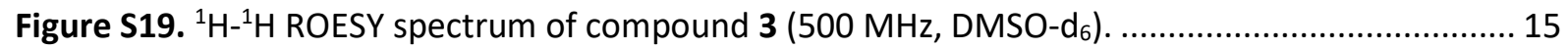

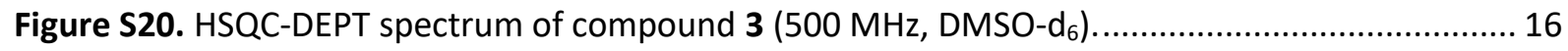

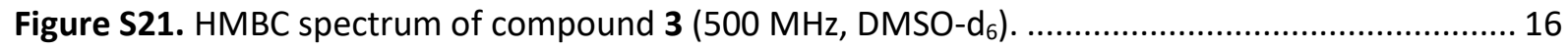

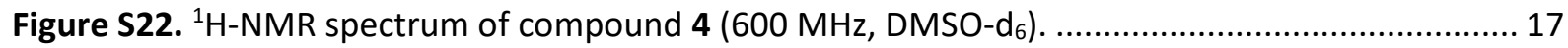

Figure S23. ${ }^{13} \mathrm{C}$-DEPTq spectrum of compound 4 (151 MHz, DMSO-d $\left.\mathrm{d}_{6}\right)$........................................ 17

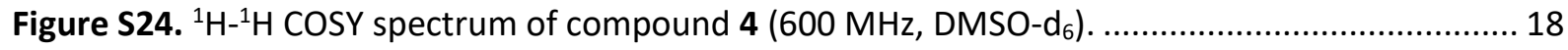

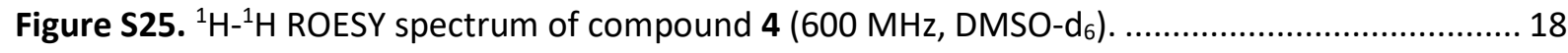

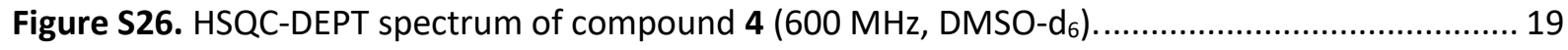

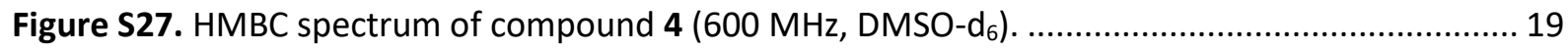

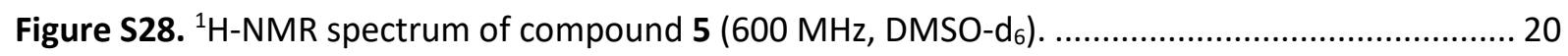

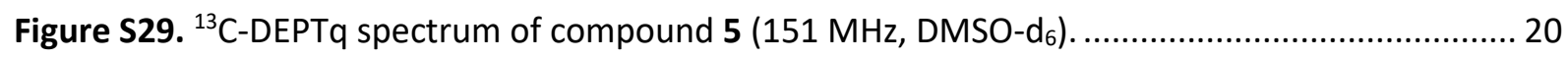

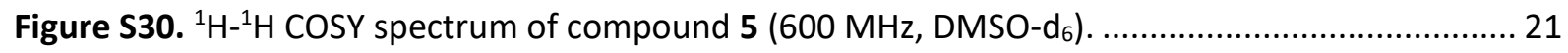

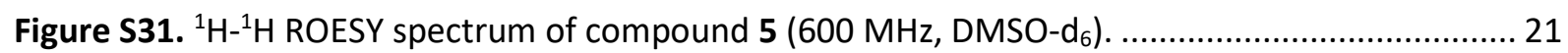




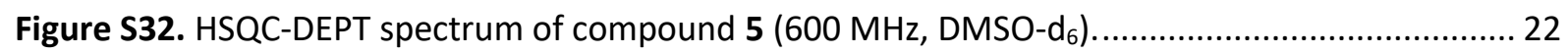

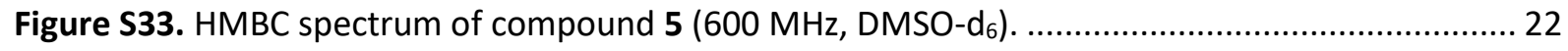

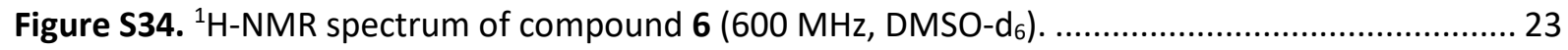

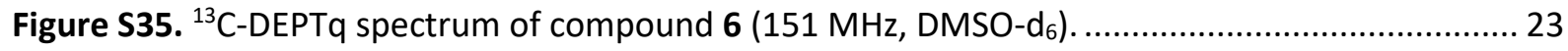

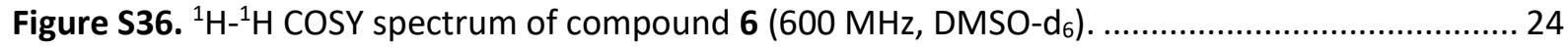

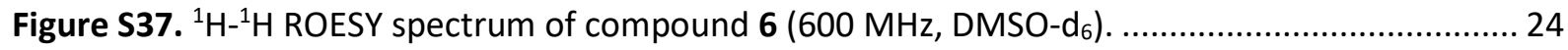

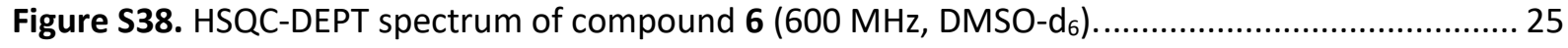

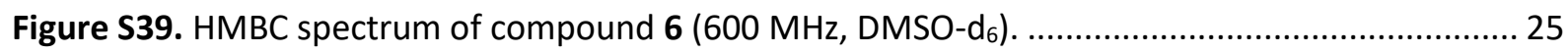

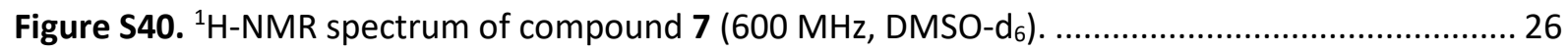

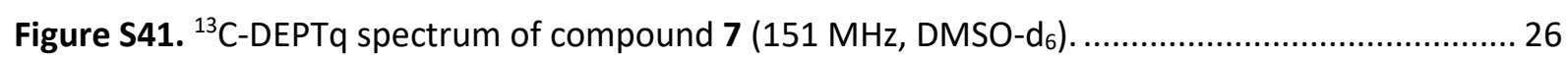

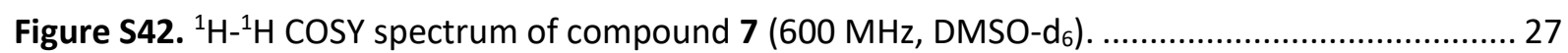

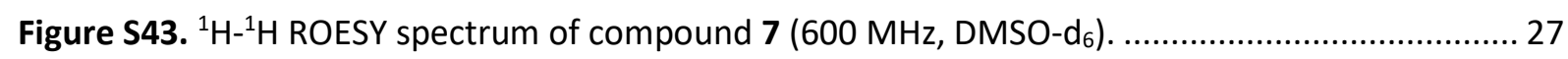

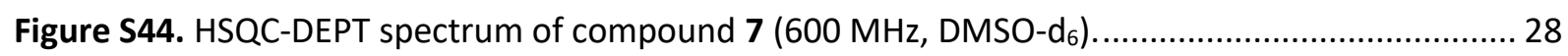

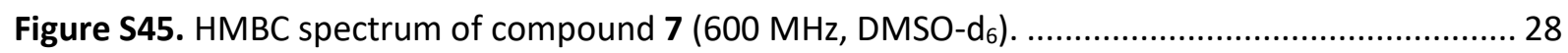

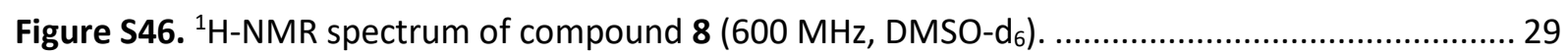

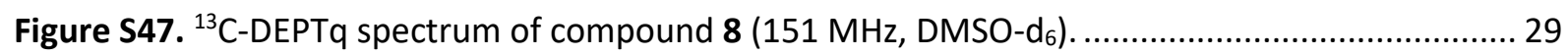

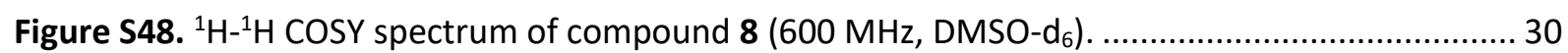

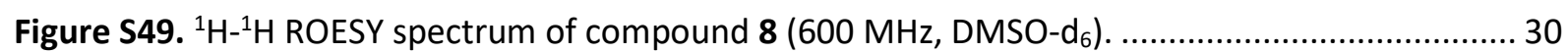

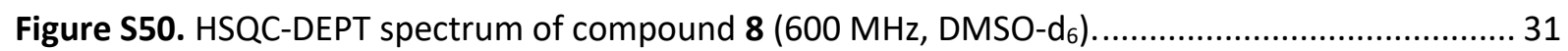

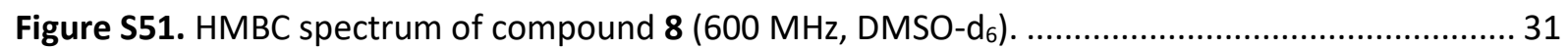

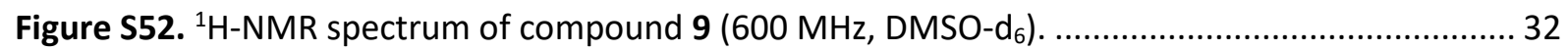

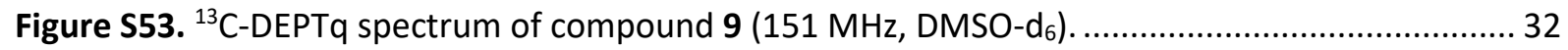

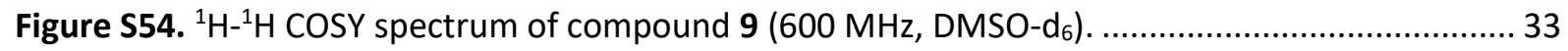

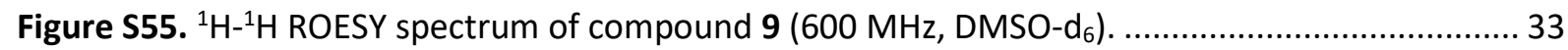

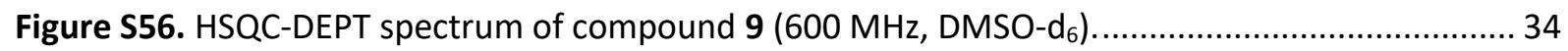

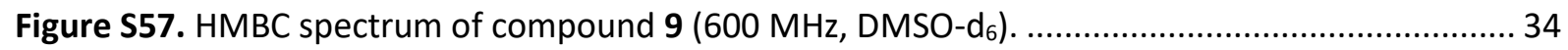

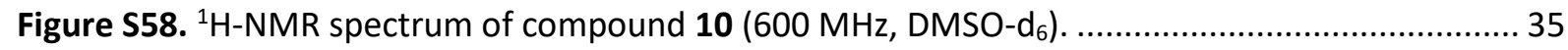

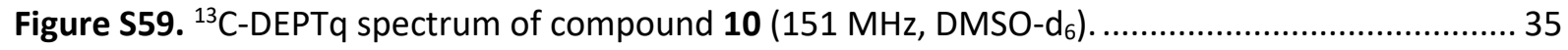

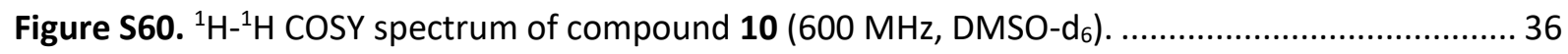

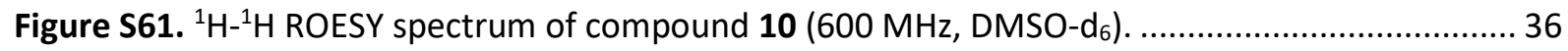

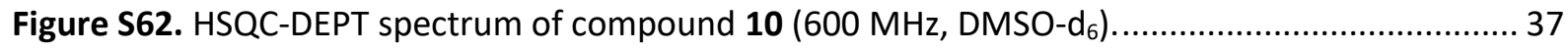

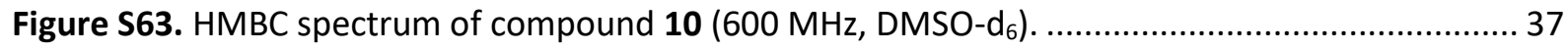



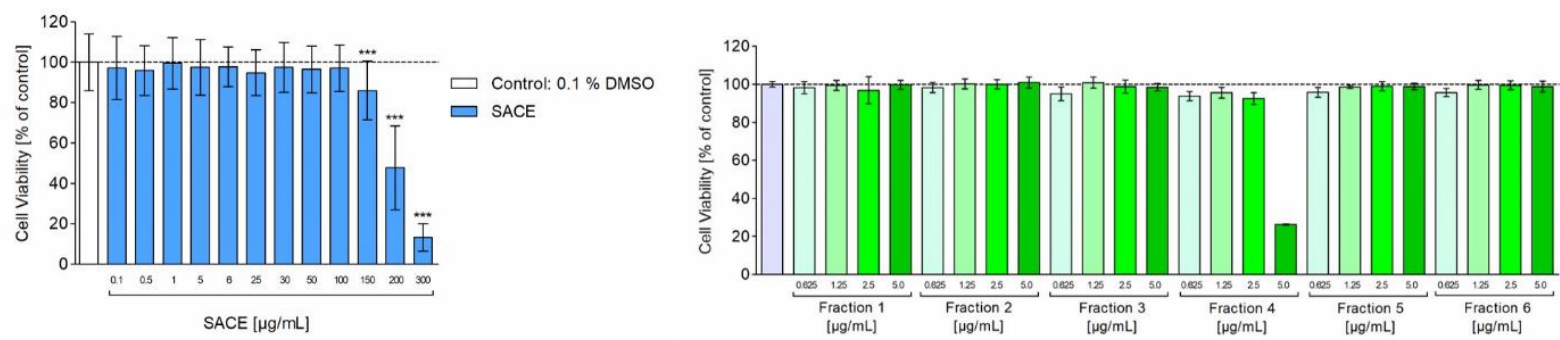

$\square$ Control: 0.1 \% DMSO
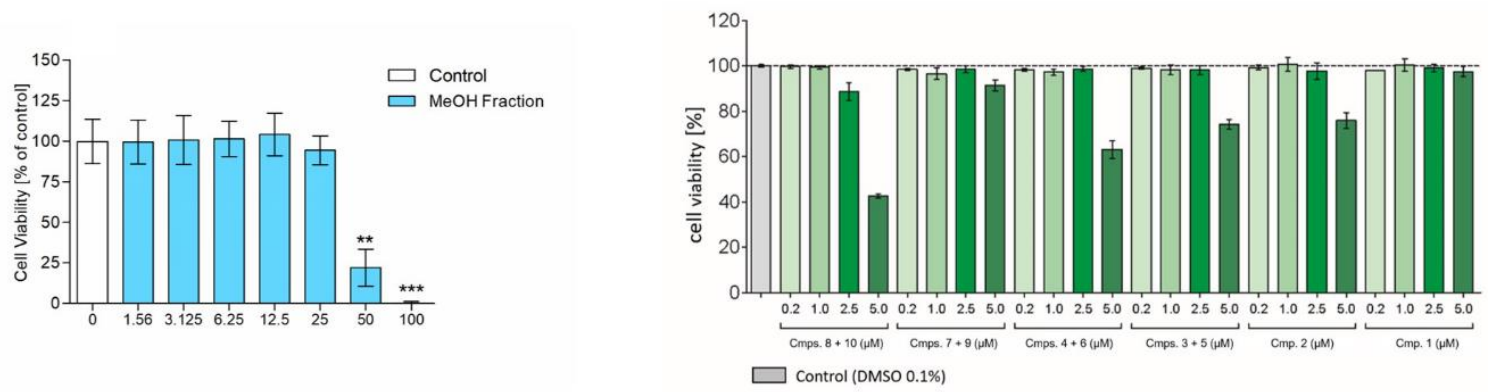

Figure S 1. Effect of the compounds 1-10 on cell viability of HaCaT cells compared to the control (DMEM incl. 0.1\% DMSO).

The results represent three independent experiments $(\mathrm{n}=3)$ and are presented as mean \pm standard deviation. ${ }^{*} \mathrm{p} \leq 0.05 \mathrm{vs}$. control, $* * \mathrm{p} \leq 0.01$ vs. control, $* * * \mathrm{p} \leq 0.001$ vs. control

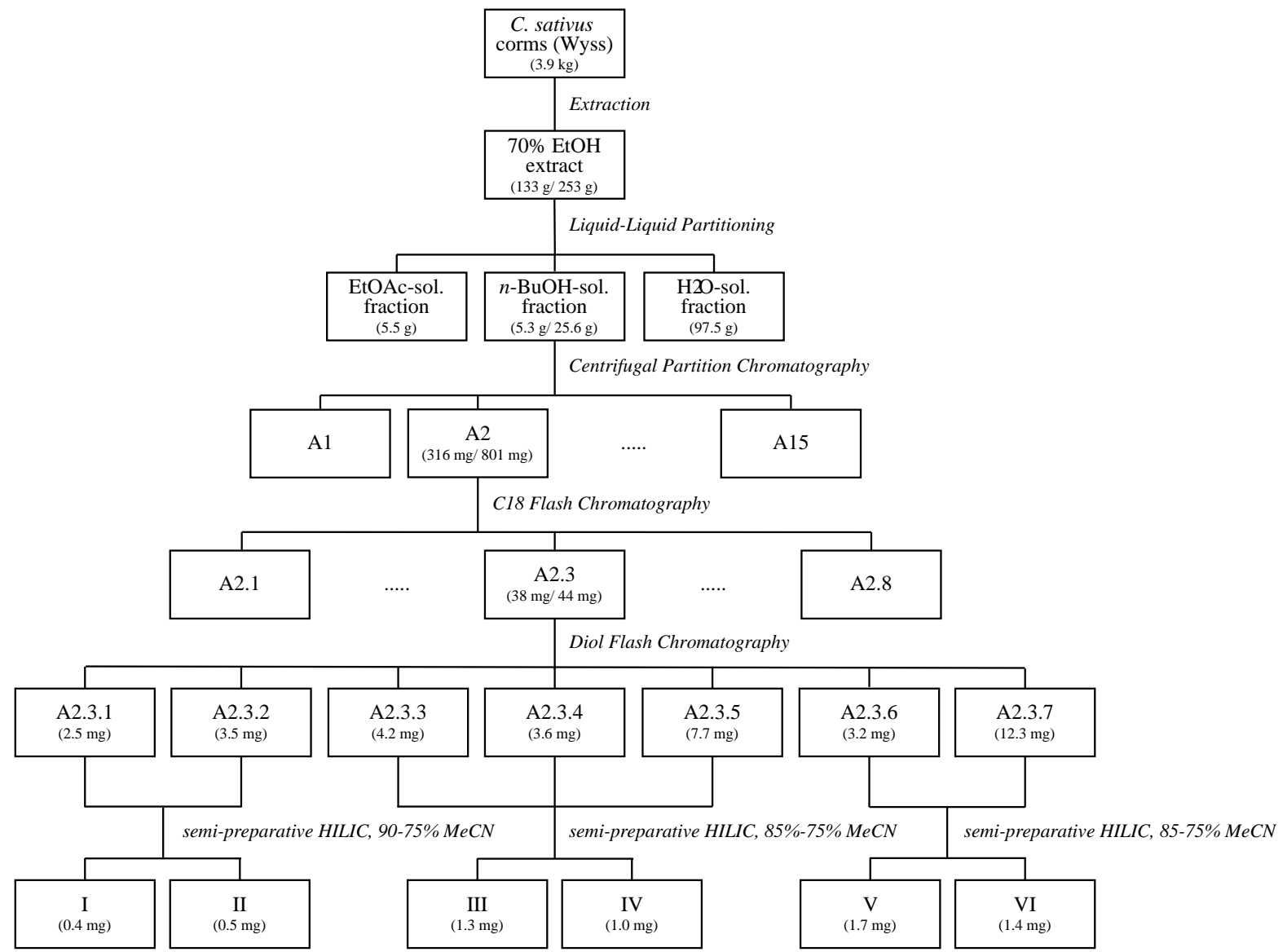

Figure S2. Isolation scheme for the isolation of substances I-VI. 


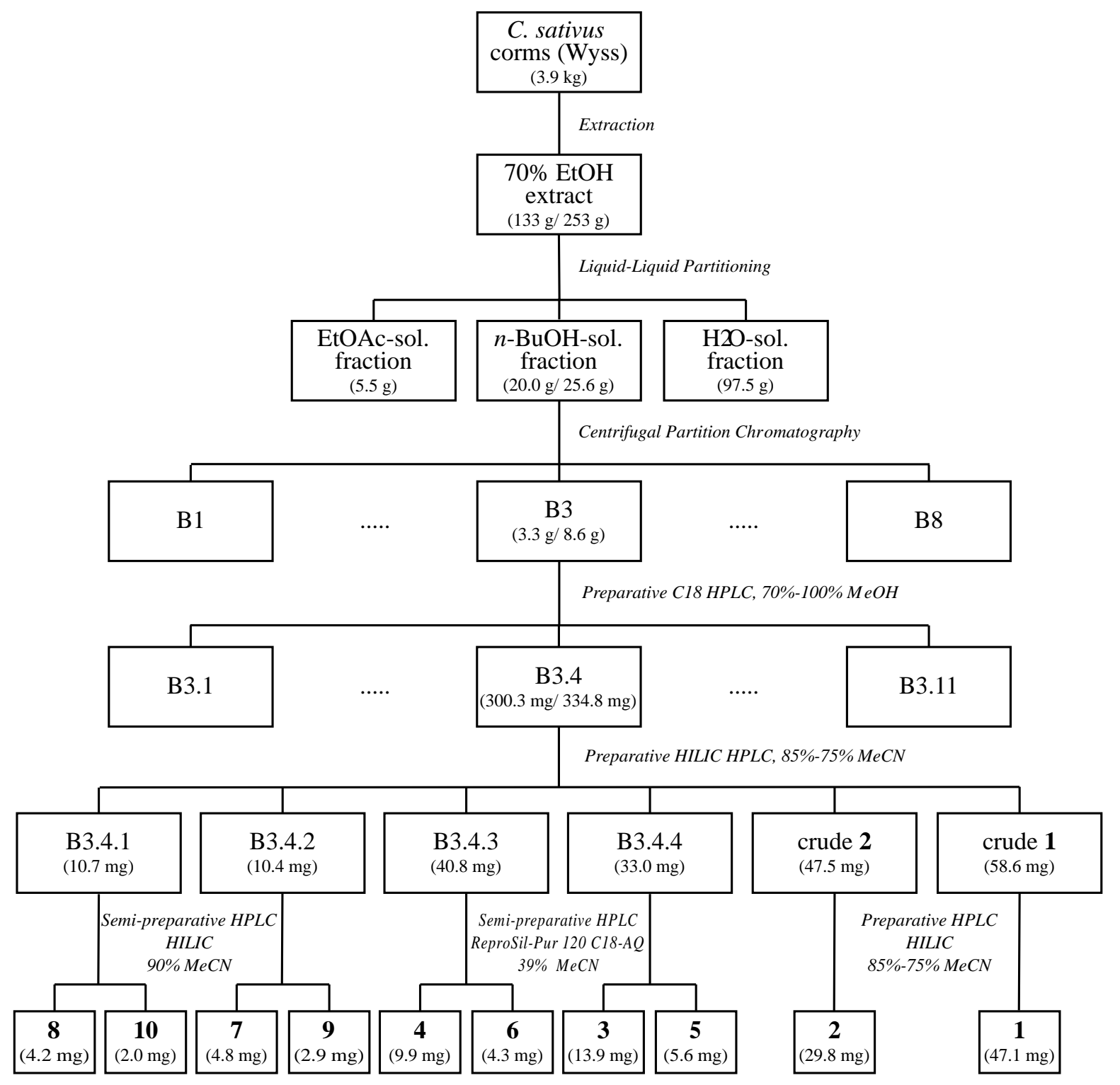

Figure S3. Isolation scheme for the isolation of compounds 1-10. 
Table S1. ${ }^{1} \mathrm{H}$ and ${ }^{13} \mathrm{C}$ NMR Spectroscopic Data of the Aglycone and Fatty Acid Residue in Compounds 1-2 (DMSO-d6; 500 $\mathrm{MHz}$ for ${ }^{1} \mathrm{H}$ and $126 \mathrm{MHz}$ for ${ }^{13} \mathrm{C}$ NMR; $\delta$ in $\mathrm{ppm}$ )

\begin{tabular}{|c|c|c|c|c|}
\hline & \multicolumn{2}{|r|}{1} & \multicolumn{2}{|r|}{2} \\
\hline position & $\delta_{\mathrm{C}}$, type & $\delta_{\mathrm{H}}(J$ in $\mathrm{Hz})$ & $\delta_{\mathrm{C}}$, type & $\delta_{\mathrm{H}}(\mathrm{J}$ in $\mathrm{Hz})$ \\
\hline \multirow{2}{*}{1} & \multirow{2}{*}{$38.3, \mathrm{CH}_{2}$} & $0.89^{\mathrm{a}}$ & \multirow{2}{*}{$38.4, \mathrm{CH}_{2}$} & $0.89^{\mathrm{a}}$ \\
\hline & & $1.52^{\mathrm{a}}$ & & $1.54^{\mathrm{a}}$ \\
\hline \multirow{2}{*}{2} & \multirow{2}{*}{ 25.7, $\mathrm{CH}_{2}$} & $1.53^{\mathrm{a}}$ & \multirow{2}{*}{ 25.6, $\mathrm{CH}_{2}$} & $1.55^{\mathrm{a}}$ \\
\hline & & $1.69^{\mathrm{a}}$ & & $1.88^{\mathrm{a}}$ \\
\hline 3 & $88.1, \mathrm{CH}$ & $3.05^{\mathrm{a}}$ & $88.2, \mathrm{CH}$ & $3.04^{\mathrm{a}}$ \\
\hline 4 & $38.8, \mathrm{C}$ & & $38.8, \mathrm{C}$ & \\
\hline 5 & $55.1, \mathrm{CH}$ & $0.69^{\mathrm{a}}$ & $55.2, \mathrm{CH}$ & $0.69^{\mathrm{a}}$ \\
\hline \multirow{2}{*}{6} & \multirow{2}{*}{ 18.0, $\mathrm{CH}_{2}$} & $1.26^{\mathrm{a}}$ & \multirow{2}{*}{ 17.9, $\mathrm{CH}_{2}$} & \\
\hline & & $1.46^{\mathrm{a}}$ & & $1.47^{\mathrm{a}}$ \\
\hline 7 & $32.6, \mathrm{CH}_{2}$ & $1.30^{\mathrm{a}}$ & 32.6, $\mathrm{CH}_{2}$ & \\
\hline 8 & $39.1, \mathrm{C}$ & $1.37^{\mathrm{a}}$ & $39.1, \mathrm{C}$ & $1.38^{\mathrm{a}}$ \\
\hline 9 & $46.1, \mathrm{CH}$ & $1.51^{\mathrm{a}}$ & $46.2, \mathrm{CH}$ & $1.51, \mathrm{~m}$ \\
\hline 10 & $36.3, \mathrm{C}$ & & $36.3, \mathrm{C}$ & \\
\hline 11 & $23.0, \mathrm{CH}_{2}$ & $1.79^{\mathrm{a}}$ & 23.0, $\mathrm{CH}_{2}$ & $1.80^{\mathrm{a}}$ \\
\hline 12 & $121.4, \mathrm{CH}$ & $5.24, \mathrm{~m}$ & $121.5, \mathrm{CH}$ & $5.24, \mathrm{~m}$ \\
\hline 13 & $143.3, \mathrm{C}$ & & $143.3, \mathrm{C}$ & \\
\hline 14 & 41.1, C & & $41.1, \mathrm{C}$ & \\
\hline \multirow[t]{2}{*}{15} & \multirow{2}{*}{$34.7, \mathrm{CH}_{2}$} & $1.28^{\mathrm{a}}$ & \multirow{2}{*}{ 34.7, $\mathrm{CH}_{2}$} & \multirow{2}{*}{$\begin{array}{l}1.30^{\mathrm{a}} \\
1.58^{\mathrm{a}}\end{array}$} \\
\hline & & $1.57^{\mathrm{a}}$ & & \\
\hline 16 & 72.7, $\mathrm{CH}$ & $4.30, \mathrm{~m}$ & $72.7, \mathrm{CH}$ & $4.31, \mathrm{~m}$ \\
\hline 17 & $48.1, \mathrm{C}$ & & $48.1, \mathrm{C}$ & \\
\hline 18 & $40.4, \mathrm{CH}$ & $2.83, \mathrm{~d}(10.7)$ & $40.4, \mathrm{CH}$ & $2.83, \mathrm{~d}(12.5)$ \\
\hline & $166 \mathrm{CH}$ & $0.99^{\mathrm{a}}$ & $467 \mathrm{CH}$ & $0.99^{\mathrm{a}}$ \\
\hline 19 & $46.6, \mathrm{CH}_{2}$ & $2.22, \mathrm{dd}(11.6,10.4)$ & $46.7, \mathrm{CH}_{2}$ & $2.22, \mathrm{dd}(11.9,11.9)$ \\
\hline 20 & $30.2, \mathrm{C}$ & & $30.2, \mathrm{C}$ & \\
\hline 21 & $35.1, \mathrm{CH}_{2}$ & $1.09^{\mathrm{a}}$ & $35.1, \mathrm{CH}_{2}$ & $1.09^{\mathrm{a}}$ \\
\hline & & $1.88^{\mathrm{a}}$ & $35.1, \mathrm{CH}_{2}$ & $1.89^{\mathrm{a}}$ \\
\hline 22 & $30.7, \mathrm{CH}_{2}$ & $\begin{array}{l}1.63^{\mathrm{a}} \\
1.81^{\mathrm{a}}\end{array}$ & $30.7, \mathrm{CH}_{2}$ & $\begin{array}{l}1.64^{\mathrm{a}} \\
1.81^{\mathrm{a}}\end{array}$ \\
\hline 23 & 27.6, $\mathrm{CH}_{3}$ & $0.97^{\mathrm{a}}$ & $27.7, \mathrm{CH}_{3}$ & $0.97^{\mathrm{a}}$ \\
\hline 24 & $16.5, \mathrm{CH}_{3}$ & $0.75, \mathrm{~s}$ & $16.5, \mathrm{CH}_{3}$ & $0.77, \mathrm{~s}$ \\
\hline 25 & $15.5, \mathrm{CH}_{3}$ & $0.86^{\mathrm{a}}$ & $15.5, \mathrm{CH}_{3}$ & $0.88^{\mathrm{a}}$ \\
\hline 26 & $16.9, \mathrm{CH}_{3}$ & $0.67^{\mathrm{a}}$ & $16.9, \mathrm{CH}_{3}$ & $0.68^{\mathrm{a}}$ \\
\hline 27 & $26.3, \mathrm{CH}_{3}$ & $1.30^{\mathrm{a}}$ & $26.3, \mathrm{CH}_{3}$ & $1.30^{\mathrm{a}}$ \\
\hline 28 & $175.1, \mathrm{C}$ & & $175.1, \mathrm{C}$ & \\
\hline 29 & $32.9, \mathrm{CH}_{3}$ & $0.82, \mathrm{~s}$ & $32.9, \mathrm{CH}_{3}$ & $0.82, \mathrm{~s}$ \\
\hline 30 & $24.2, \mathrm{CH}_{3}$ & $0.90^{\mathrm{a}}$ & $24.2, \mathrm{CH}_{3}$ & $0.89^{\mathrm{a}}$ \\
\hline $\mathrm{C} 1$ ' & $170.8, \mathrm{C}$ & & $170.8, \mathrm{C}$ & \\
\hline $\mathrm{C} 2$, & $38.3, \mathrm{CH}_{2}$ & $2.48^{\mathrm{a}}$ & $38.3, \mathrm{CH}_{2}$ & $2.49^{\mathrm{a}}$ \\
\hline $\mathrm{C} 2$ & $38.5, \mathrm{CH}_{2}$ & 2.70, dd $(15.6,3.7)$ & $38.3, \mathrm{CH}_{2}$ & $2.71, \mathrm{dd}(15.6,4.3)$ \\
\hline C3' & $72.4, \mathrm{CH}$ & $3.90, \mathrm{tt}(5.2,5.2)$ & $72.3, \mathrm{CH}$ & $3.90, \mathrm{tt}(5.5,5.5)$ \\
\hline $\mathrm{C} 4$ ' & $34.4, \mathrm{CH}_{2}$ & $1.53^{\mathrm{a}}$ & $34.3, \mathrm{CH}_{2}$ & $1.53^{\mathrm{a}}$ \\
\hline $\mathrm{C} 5$ & $24.5, \mathrm{CH}_{2}$ & $1.32^{\mathrm{a}}$ & $24.5, \mathrm{CH}_{2}$ & $1.33^{\mathrm{a}}$ \\
\hline C6' & $28.6, \mathrm{CH}_{2}$ & $1.21^{\mathrm{a}}$ & 28.6, $\mathrm{CH}_{2}$ & $1.22^{\mathrm{a}}$ \\
\hline $\mathrm{C} 7$ & $28.6, \mathrm{CH}_{2}$ & $1.22^{\mathrm{a}}$ & $28.6, \mathrm{CH}_{2}$ & $1.24^{\mathrm{a}}$ \\
\hline $\mathrm{C} 8$, & $23.3, \mathrm{CH}_{2}$ & $1.43^{\mathrm{a}}$ & $23.3, \mathrm{CH}_{2}$ & $1.43^{\mathrm{a}}$ \\
\hline C9' & $41.8, \mathrm{CH}_{2}$ & $2.37, \mathrm{t}(6.9)$ & $41.8, \mathrm{CH}_{2}$ & $2.37, \mathrm{t}(7.3)$ \\
\hline $\mathrm{C} 10^{\prime}$ & $210.6, \mathrm{C}$ & & $210.6, \mathrm{C}$ & \\
\hline $\mathrm{C} 11$ & $41.8, \mathrm{CH}_{2}$ & $2.37, \mathrm{t}(6.9)$ & $41.8, \mathrm{CH}_{2}$ & $2.37, \mathrm{t}(7.3)$ \\
\hline C12' & $23.3, \mathrm{CH}_{2}$ & $1.43^{\mathrm{a}}$ & 23.3, $\mathrm{CH}_{2}$ & $1.43^{\mathrm{a}}$ \\
\hline C13' & $28.7, \mathrm{CH}_{2}$ & $1.20^{\mathrm{a}}$ & $28.7, \mathrm{CH}_{2}$ & $1.21^{\mathrm{a}}$ \\
\hline C14' & $25.6, \mathrm{CH}_{2}$ & $1.25^{\mathrm{a}}$ & 25.6, $\mathrm{CH}_{2}$ & $1.26^{\mathrm{a}}$ \\
\hline $\mathrm{C} 15$ & $29.1, \mathrm{CH}_{2}$ & $1.47^{\mathrm{a}}$ & 29.1, $\mathrm{CH}_{2}$ & $1.47^{\mathrm{a}}$ \\
\hline C16' & 6 & $3.30^{\mathrm{a}}$ & $H_{2}$ & $3.30^{\mathrm{a}}$ \\
\hline C16 & $60.4, \mathrm{CH}_{2}$ & $3.52^{\mathrm{a}}$ &,$+ \mathrm{CH}_{2}$ & $3.51^{\mathrm{a}}$ \\
\hline
\end{tabular}


Table S2. ${ }^{1} \mathrm{H}$ and ${ }^{13} \mathrm{C}$ NMR Spectroscopic Data of the Glycosidic Moiety in Compounds 1-2 (DMSO-d6; $500 \mathrm{MHz}$ for ${ }^{1} \mathrm{H}$ and $126 \mathrm{MHz}$ for ${ }^{13} \mathrm{C}$ NMR; $\delta$ in ppm)

\begin{tabular}{|c|c|c|c|c|}
\hline & & & & 2 \\
\hline$\overline{\text { position }}$ & $\delta_{\mathrm{C}}$, type & $\delta_{\mathrm{H}}(J$ in $\mathrm{Hz})$ & $\delta_{\mathrm{C}}$, type & $\delta_{\mathrm{H}}(J$ in $\mathrm{Hz})$ \\
\hline Sugar $\mathrm{m}$ & iety at 3 & & & \\
\hline GalA & & & & \\
\hline 1 & - & & $105.5, \mathrm{CH}$ & $4.16, \mathrm{~d}(4.9)$ \\
\hline 2 & - & & $70.6, \mathrm{CH}$ & $3.31^{\mathrm{a}}$ \\
\hline 3 & - & & $73.1, \mathrm{CH}$ & $3.32^{\mathrm{a}}$ \\
\hline 4 & - & & $70.0, \mathrm{CH}$ & $3.87^{\mathrm{a}}$ \\
\hline 5 & - & & $73.8, \mathrm{CH}$ & 3.96 , br s \\
\hline 6 & - & & $170.5, \mathrm{C}$ & \\
\hline GlcA & & & & \\
\hline 1 & 105.6, CH & $4.24, \mathrm{~d}(7.0)$ & - & \\
\hline 2 & $73.7, \mathrm{CH}$ & $2.99^{\mathrm{a}}$ & - & \\
\hline 3 & $76.2, \mathrm{CH}$ & $3.14^{\mathrm{a}}$ & - & \\
\hline 4 & $71.6, \mathrm{CH}$ & $3.27^{\mathrm{a}}$ & - & \\
\hline 5 & $75.5, \mathrm{CH}$ & $3.56^{\mathrm{a}}$ & - & \\
\hline 6 & $170.8, \mathrm{C}$ & & - & \\
\hline Sugar m & iety at 28 & & & \\
\hline Fuc & & & & \\
\hline 1 & $93.0, \mathrm{CH}$ & $5.30^{\mathrm{a}}$ & 93.0, CH & $5.31^{\mathrm{a}}$ \\
\hline 2 & 74.7, CH & $3.47^{\mathrm{a}}$ & $74.7, \mathrm{CH}$ & $3.47^{\mathrm{a}}$ \\
\hline 3 & $71.6, \mathrm{CH}$ & $3.81^{\mathrm{a}}$ & $71.6, \mathrm{CH}$ & $3.81^{\mathrm{a}}$ \\
\hline 4 & $73.5, \mathrm{CH}$ & 4.92 , br s & $73.5, \mathrm{CH}$ & 4.92 , br s \\
\hline 5 & $68.8, \mathrm{CH}$ & $3.85^{\mathrm{a}}$ & $68.8, \mathrm{CH}$ & $3.85^{\mathrm{a}}$ \\
\hline 6 & $16.0, \mathrm{CH}_{3}$ & $0.92, \mathrm{~d}(5.8)$ & $16.0, \mathrm{CH}_{3}$ & $0.92^{\mathrm{a}}$ \\
\hline Rha3 & & & & \\
\hline 1 & $98.5, \mathrm{CH}$ & $5.31^{\mathrm{a}}$ & $98.5, \mathrm{CH}$ & $5.32^{\mathrm{a}}$ \\
\hline 2 & $78.2, \mathrm{CH}$ & $3.83^{\mathrm{a}}$ & $78.2, \mathrm{CH}$ & $3.83^{\mathrm{a}}$ \\
\hline 3 & $70.3, \mathrm{CH}$ & $3.71^{\mathrm{a}}$ & $70.3, \mathrm{CH}$ & $3.70^{\mathrm{a}}$ \\
\hline 4 & $82.1, \mathrm{CH}$ & $3.36^{\mathrm{a}}$ & $82.0, \mathrm{CH}$ & $3.36^{\mathrm{a}}$ \\
\hline 5 & $67.0, \mathrm{CH}$ & $3.55^{\mathrm{a}}$ & $67.0, \mathrm{CH}$ & $3.56^{\mathrm{a}}$ \\
\hline 6 & $18.0, \mathrm{CH}_{3}$ & $1.18^{\mathrm{a}}$ & $18.0, \mathrm{CH}_{3}$ & $1.18, \mathrm{~d}(6.1)$ \\
\hline Xyl & & & & \\
\hline 1 & $104.7, \mathrm{CH}$ & $4.48, \mathrm{~d}(7.6)$ & $104.7, \mathrm{CH}$ & $4.48, \mathrm{~d}(7.6)$ \\
\hline 2 & 74.6, CH & $2.99^{\mathrm{a}}$ & 74.6, CH & $2.99^{\mathrm{a}}$ \\
\hline 3 & $76.3, \mathrm{CH}$ & $3.17^{\mathrm{a}}$ & $76.3, \mathrm{CH}$ & $3.17^{\mathrm{a}}$ \\
\hline 4 & $69.7, \mathrm{CH}$ & $3.24^{\mathrm{a}}$ & $69.7, \mathrm{CH}$ & $3.24^{\mathrm{a}}$ \\
\hline 5 & 65.7, $\mathrm{CH}_{2}$ & $3.15^{\mathrm{a}}$ & $65.8, \mathrm{CH}_{2}$ & $3.17^{\mathrm{a}}$ \\
\hline & & $3.65^{\mathrm{a}}$ & & $3.65^{\mathrm{a}}$ \\
\hline Ara & & & & \\
\hline 1 & $102.0, \mathrm{CH}$ & $4.57, \mathrm{~d}(4.3)$ & $102.0, \mathrm{CH}$ & $4.57, \mathrm{~d}(4.9)$ \\
\hline 2 & 78.2, CH & $3.68^{\mathrm{a}}$ & $78.2, \mathrm{CH}$ & $3.68^{\mathrm{a}}$ \\
\hline 3 & $71.2, \mathrm{CH}$ & $3.63^{\mathrm{a}}$ & $71.2, \mathrm{CH}$ & $3.63^{\mathrm{a}}$ \\
\hline 4 & $66.2, \mathrm{CH}$ & $3.70^{\mathrm{a}}$ & $66.2, \mathrm{CH}$ & $3.70^{\mathrm{a}}$ \\
\hline 5 & $638 \mathrm{CH}_{2}$ & $3.35^{\mathrm{a}}$ & $638 \mathrm{CH}_{2}$ & $3.35^{\mathrm{a}}$ \\
\hline & $03.8, \mathrm{CH}_{2}$ & $3.66^{\mathrm{a}}$ & $03.8, \mathrm{CH}_{2}$ & $3.67^{\mathrm{a}}$ \\
\hline Gal & & & & \\
\hline 1 & 104.0, CH & $4.33, \mathrm{~d}(7.6)$ & $104.0, \mathrm{CH}$ & $4.33, \mathrm{~d}(7.3)$ \\
\hline 2 & $71.1, \mathrm{CH}$ & $3.35^{\mathrm{a}}$ & 71.1, CH & $3.35^{\mathrm{a}}$ \\
\hline 3 & $73.4, \mathrm{CH}$ & $3.31^{\mathrm{a}}$ & $73.4, \mathrm{CH}$ & $3.31^{\mathrm{a}}$ \\
\hline 4 & $67.8, \mathrm{CH}$ & $3.67^{\mathrm{a}}$ & $67.7, \mathrm{CH}$ & $3.67^{\mathrm{a}}$ \\
\hline 5 & 75.4, CH & $3.37^{\mathrm{a}}$ & $75.4, \mathrm{CH}$ & $3.38^{\mathrm{a}}$ \\
\hline 6 & $600 \mathrm{CH}_{2}$ & $3.50^{\mathrm{a}}$ & $600 \mathrm{CH}_{3}$ & $3.50^{\mathrm{a}}$ \\
\hline & & $3.58^{\mathrm{a}}$ & & $3.59^{\mathrm{a}}$ \\
\hline Sugar m & iety at C3, & & & \\
\hline Rha2 & & & & \\
\hline 1 & $98.8, \mathrm{CH}$ & 4.70 , br s & $98.8, \mathrm{CH}$ & 4.70 , br s \\
\hline 2 & $70.9, \mathrm{CH}$ & $3.54^{\mathrm{a}}$ & $70.9, \mathrm{CH}$ & $3.54^{\mathrm{a}}$ \\
\hline 3 & $70.8, \mathrm{CH}$ & $3.40^{\mathrm{a}}$ & $70.8, \mathrm{CH}$ & $3.40^{\mathrm{a}}$ \\
\hline 4 & 71.9, CH & $3.18^{\mathrm{a}}$ & $71.9, \mathrm{CH}$ & $3.18^{\mathrm{a}}$ \\
\hline 5 & $69.0, \mathrm{CH}$ & $3.44^{\mathrm{a}}$ & $69.0, \mathrm{CH}$ & $3.44^{\mathrm{a}}$ \\
\hline 6 & $17.8, \mathrm{CH}_{3}$ & $1.12, \mathrm{~d}(5.8)$ & $17.8, \mathrm{CH}_{3}$ & $1.12, \mathrm{~d}(6.1)$ \\
\hline Sugar m & iety at C16' & & & \\
\hline Rha1 & & & & \\
\hline 1 & $100.0, \mathrm{CH}$ & 4.50 , br s & $100.0, \mathrm{CH}$ & $4.51, \mathrm{~d}(0.9)^{\mathrm{a}}$ \\
\hline 2 & $70.6, \mathrm{CH}$ & $3.57^{\mathrm{a}}$ & $70.6, \mathrm{CH}$ & $3.57^{\mathrm{a}}$ \\
\hline 3 & $70.8, \mathrm{CH}$ & $3.39^{\mathrm{a}}$ & $70.8, \mathrm{CH}$ & $3.38^{\mathrm{a}}$ \\
\hline 4 & $72.0, \mathrm{CH}$ & $3.16^{\mathrm{a}}$ & $72.0, \mathrm{CH}$ & $3.16^{\mathrm{a}}$ \\
\hline 5 & $68.4, \mathrm{CH}$ & $3.36^{\mathrm{a}}$ & $68.4, \mathrm{CH}$ & $3.36^{\mathrm{a}}$ \\
\hline 6 & $18.0, \mathrm{CH}_{3}$ & $1.12, \mathrm{~d}(5.8)$ & $17.8, \mathrm{CH}_{3}$ & $1.12, \mathrm{~d}(6.1)$ \\
\hline
\end{tabular}




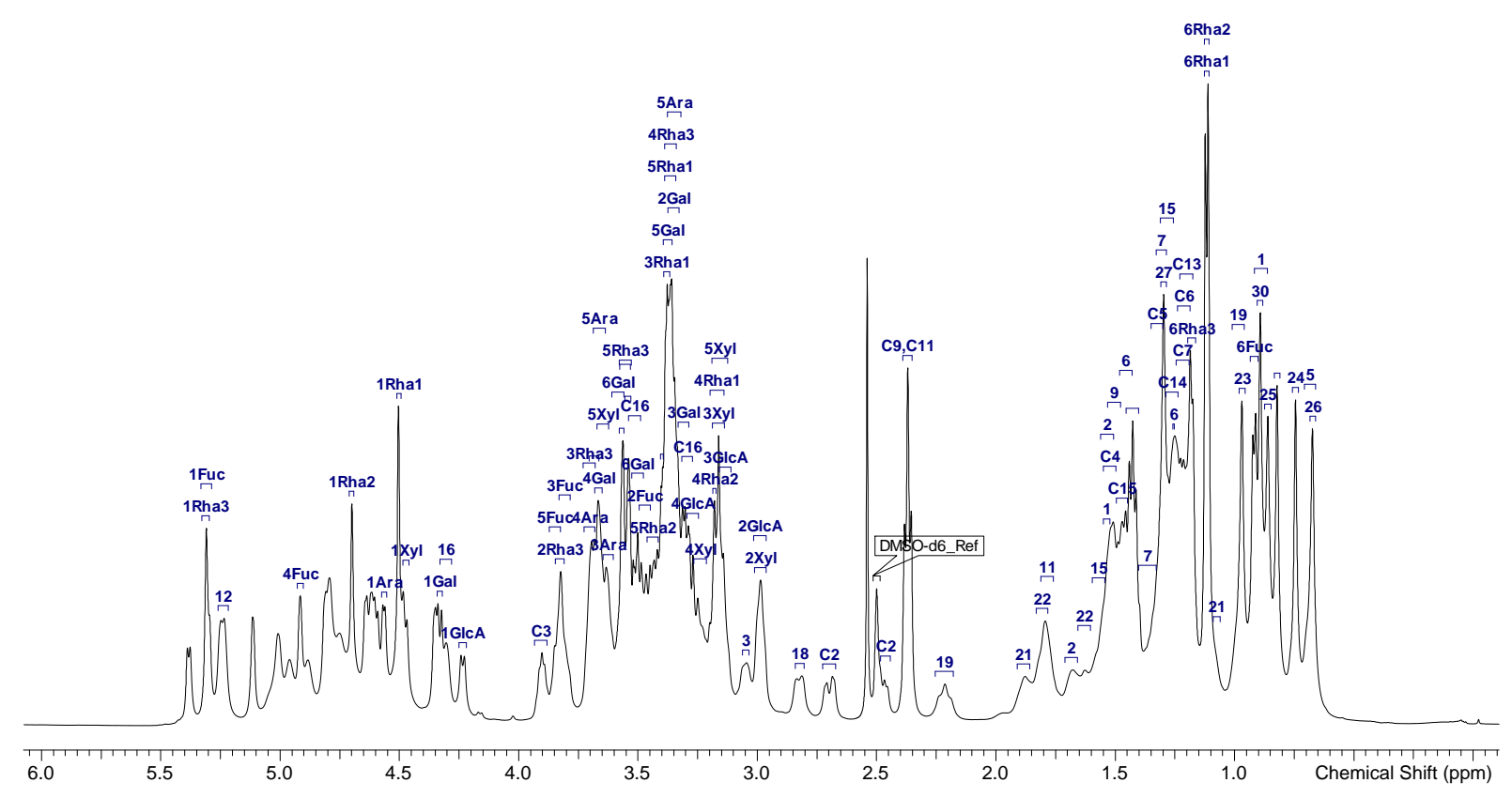

Figure S4. ${ }^{1} \mathrm{H}-\mathrm{NMR}$ spectrum of compound 1 (500 MHz, DMSO-d 6 ).

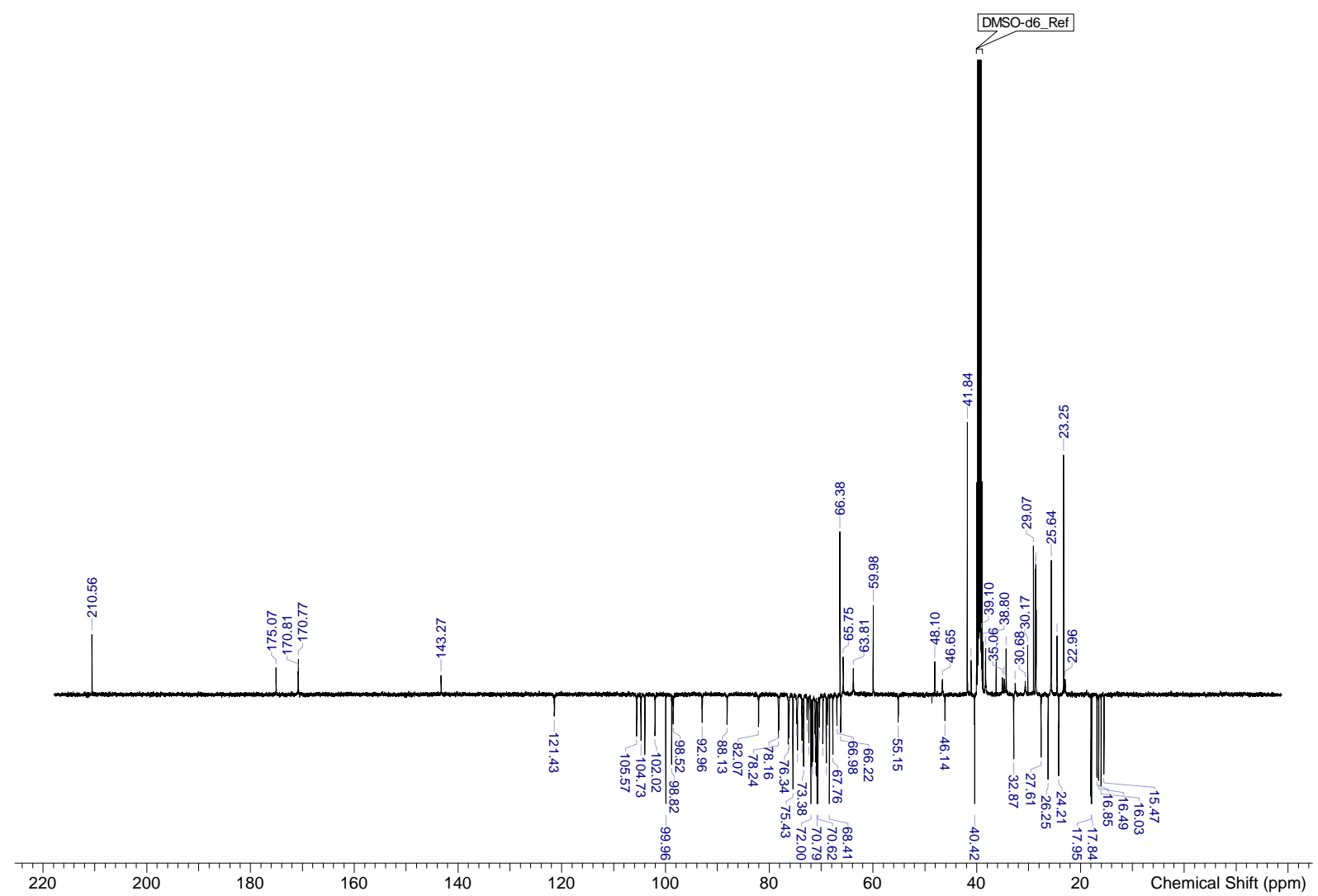

Figure S5. ${ }^{13} \mathrm{C}$-DEPTq spectrum of compound $\mathbf{1}\left(126 \mathrm{MHz}, \mathrm{DMSO}-\mathrm{d}_{6}\right)$. 


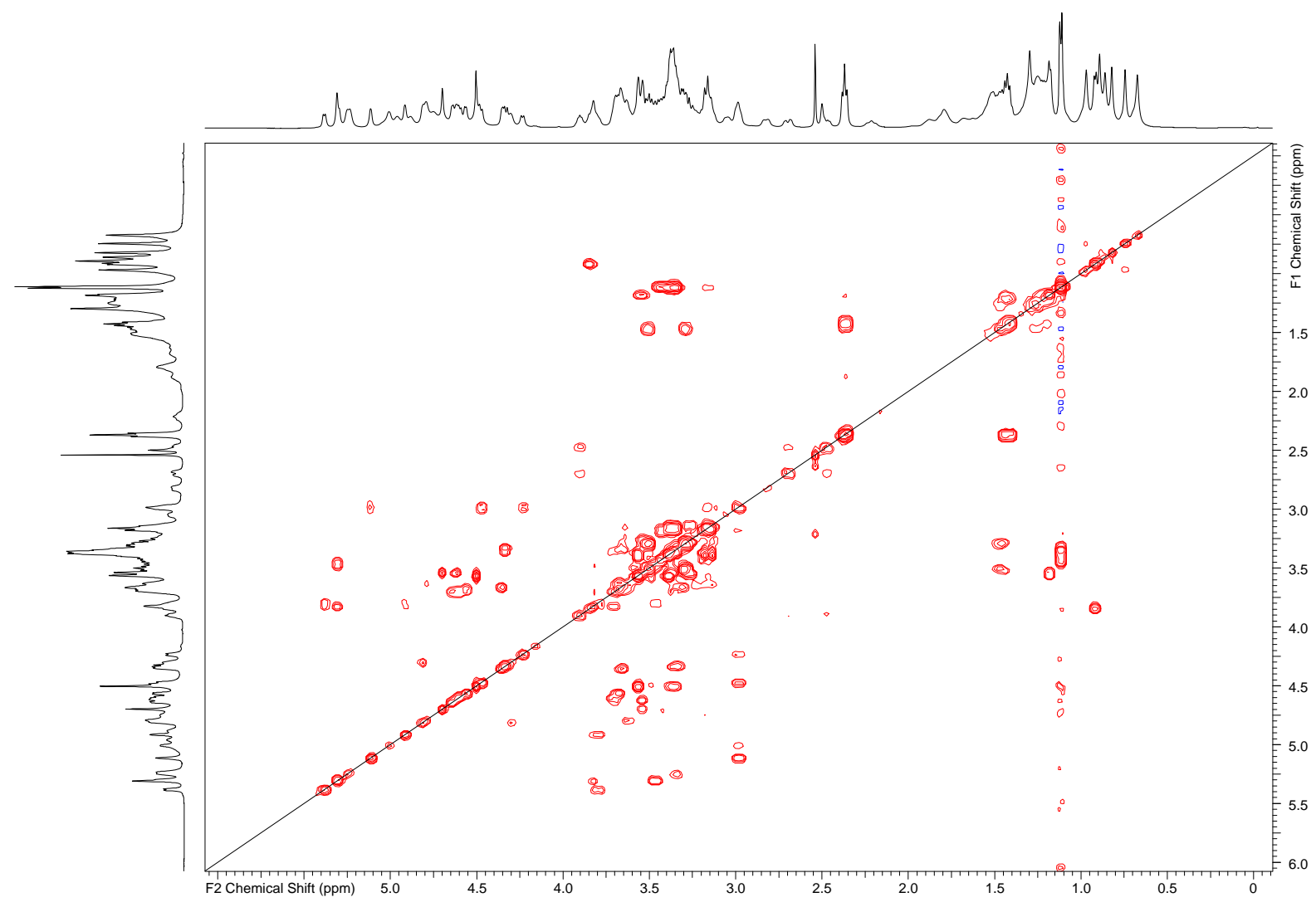

Figure S6. ${ }^{1} \mathrm{H}-{ }^{1} \mathrm{H}$ COSY spectrum of compound 1 (500 MHz, DMSO-d 6 ).

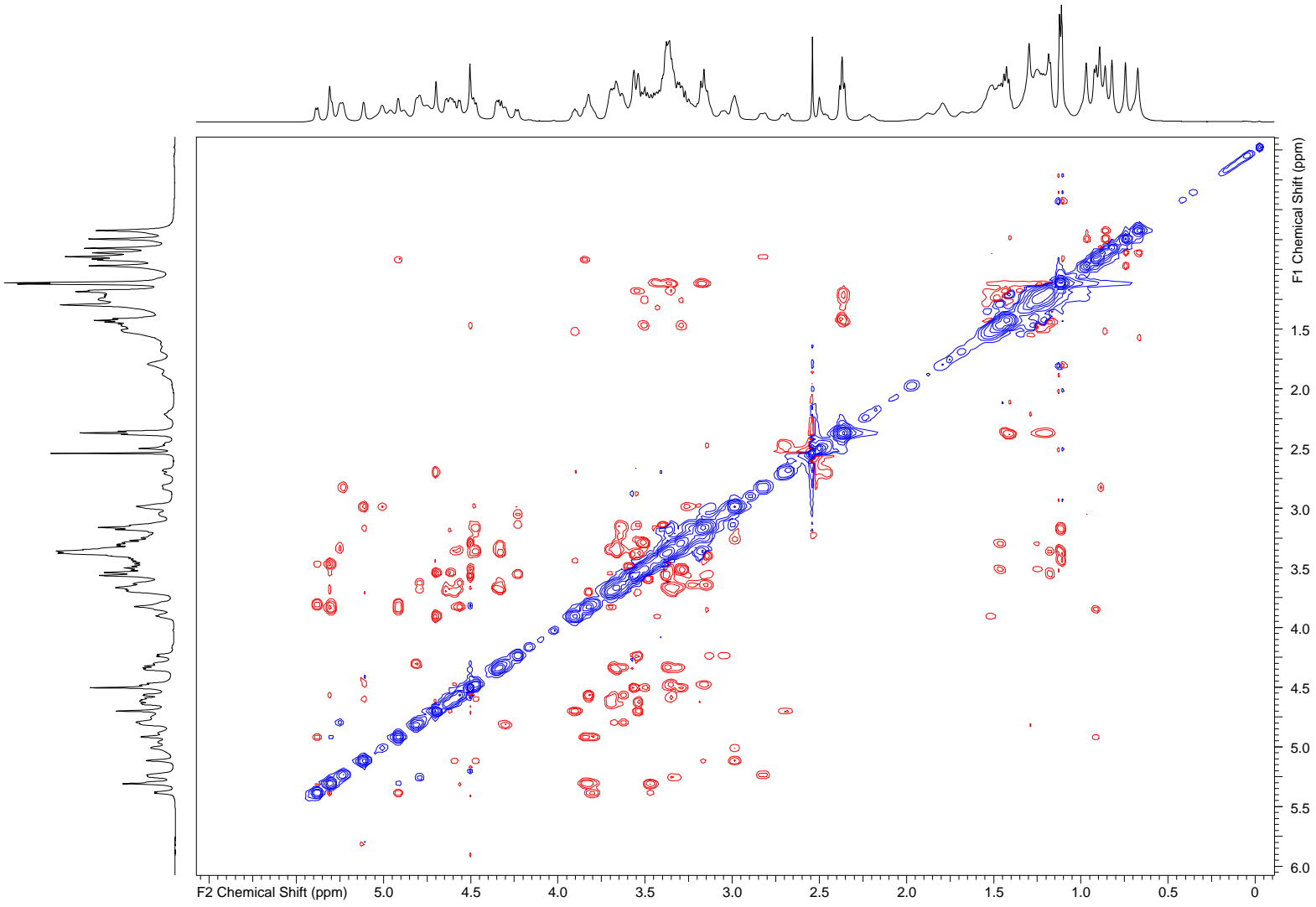

Figure S7. ${ }^{1} \mathrm{H}^{1}{ }^{1} \mathrm{H}$ ROESY spectrum of compound 1 (500 MHz, DMSO-d 6 ). 


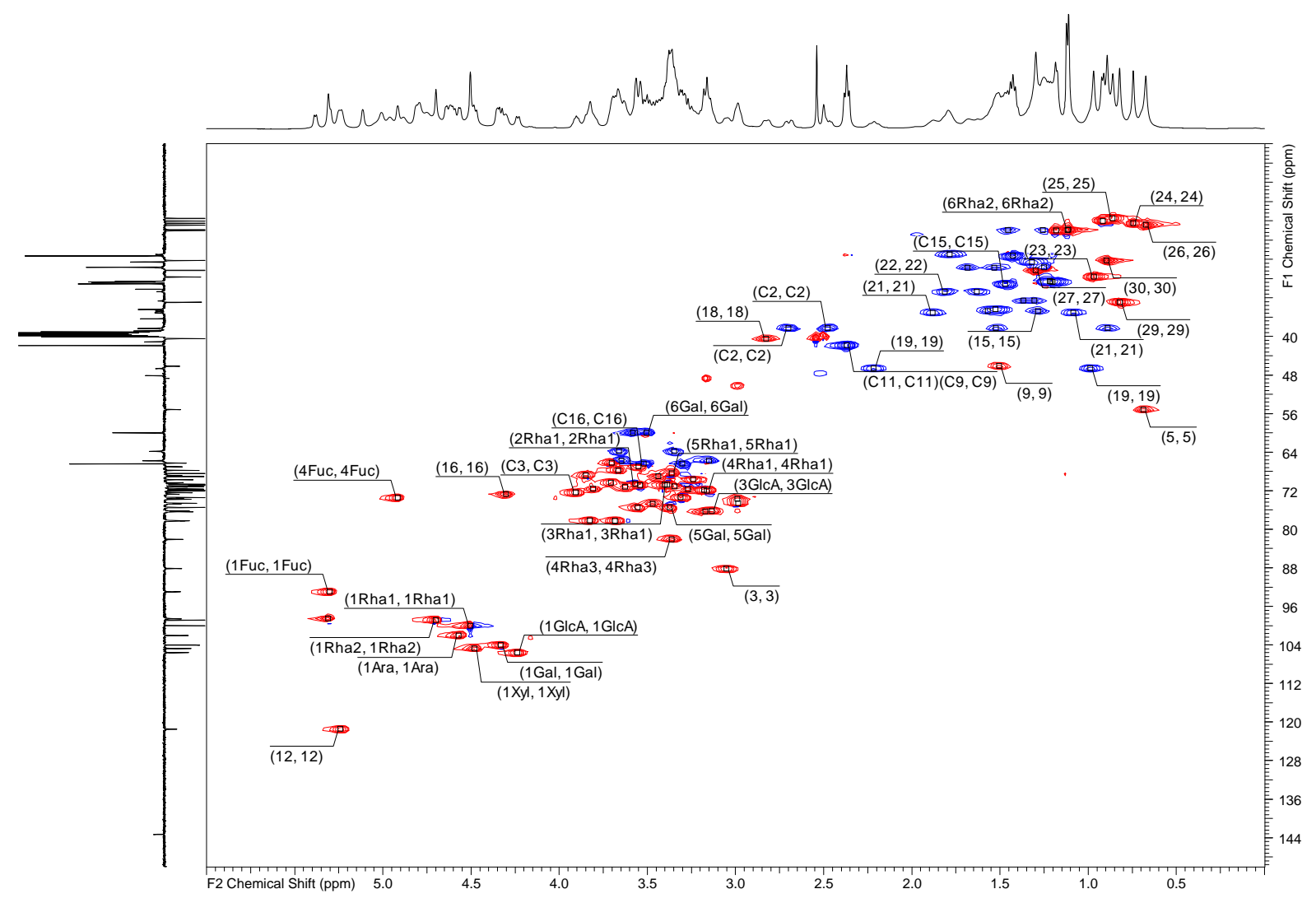

Figure S8. HSQC-DEPT spectrum of compound 1 (500 MHz, DMSO-d 6 ).

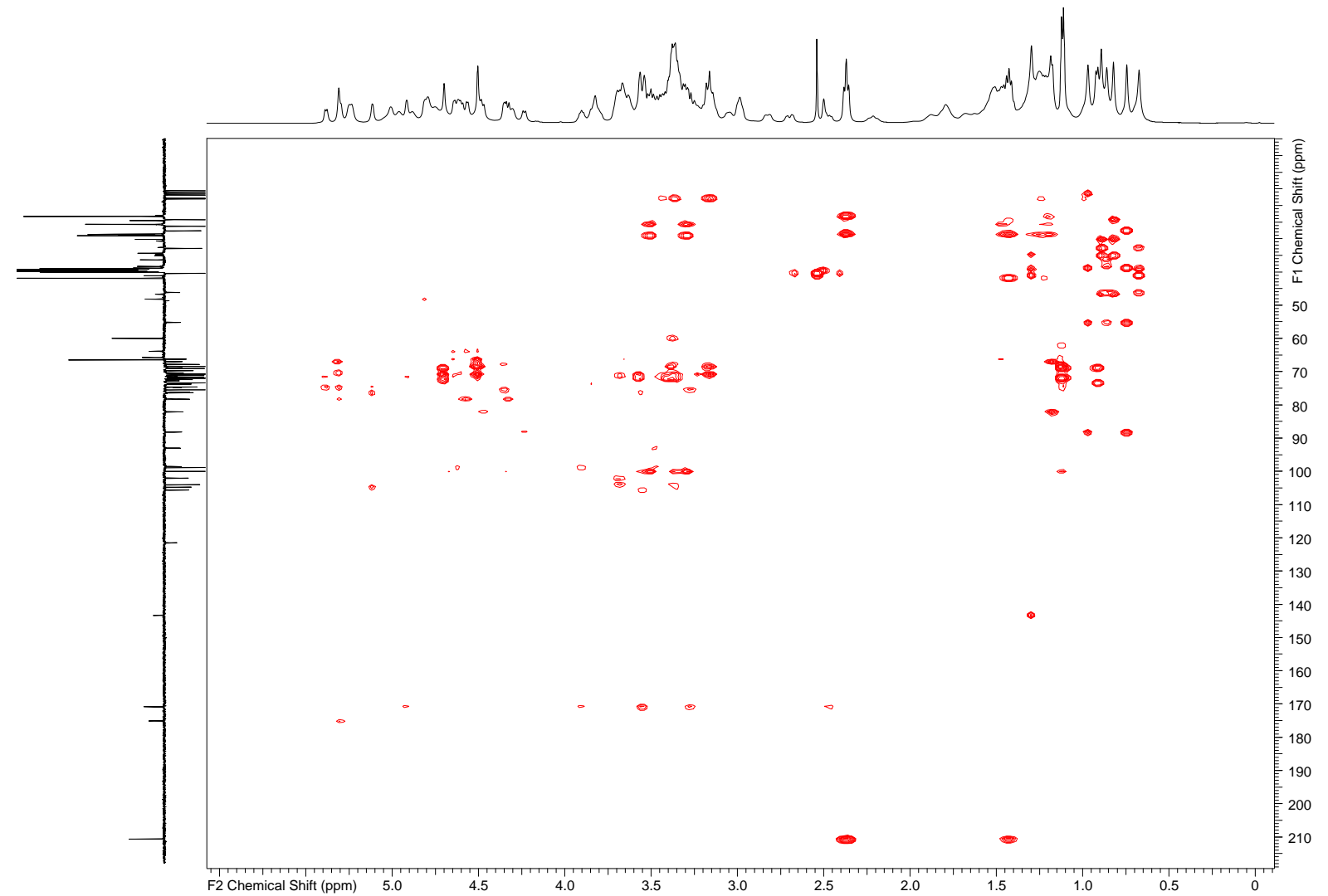

Figure S9. HMBC spectrum of compound 1 (500 MHz, DMSO-d $)$. 


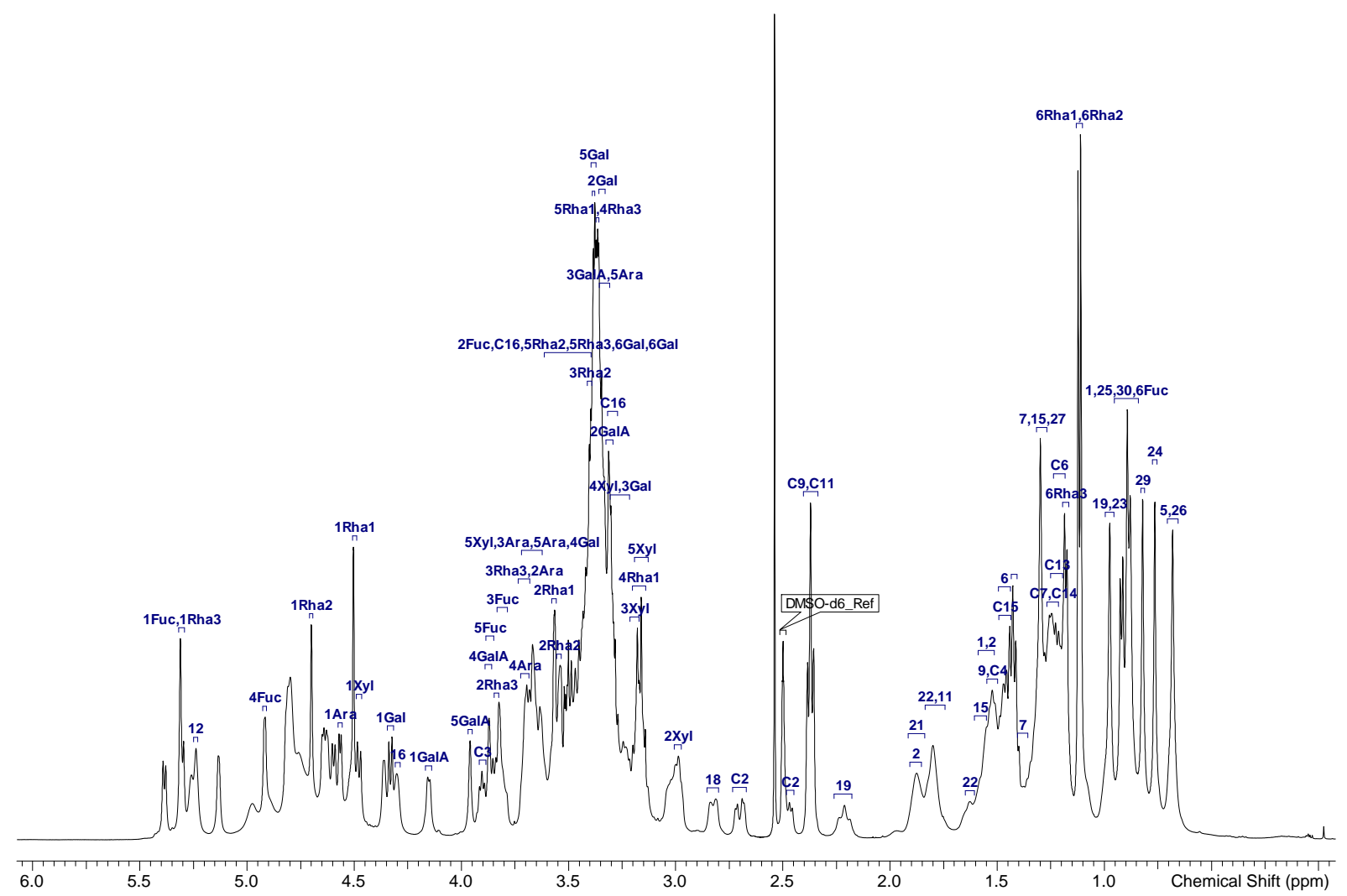

Figure S10. ${ }^{1} \mathrm{H}-\mathrm{NMR}$ spectrum of compound 2 (500 MHz, DMSO-d6).

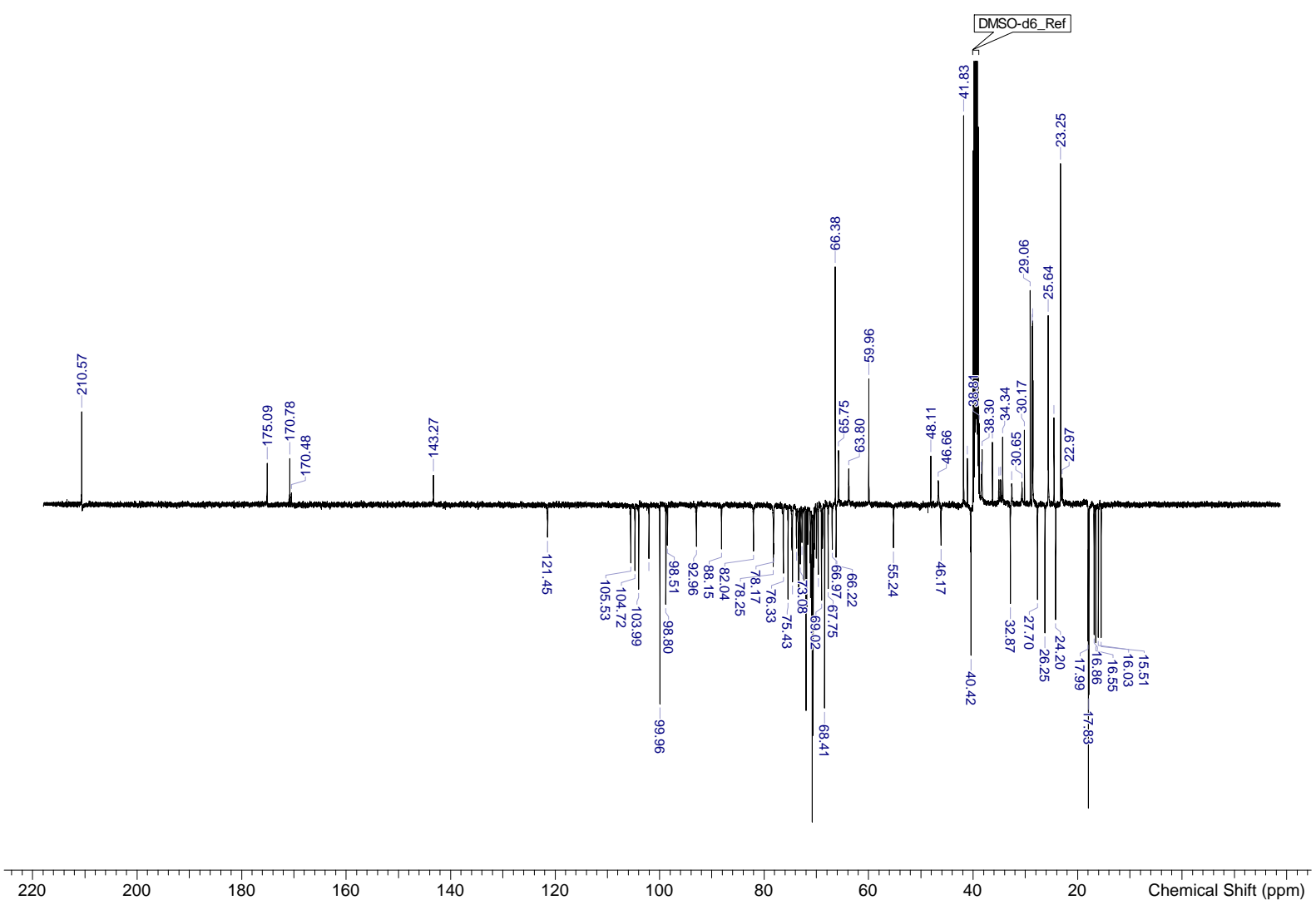

Figure S11. ${ }^{13} \mathrm{C}-\mathrm{DEPTq}$ spectrum of compound 2 (126 MHz, DMSO-d $)$. 


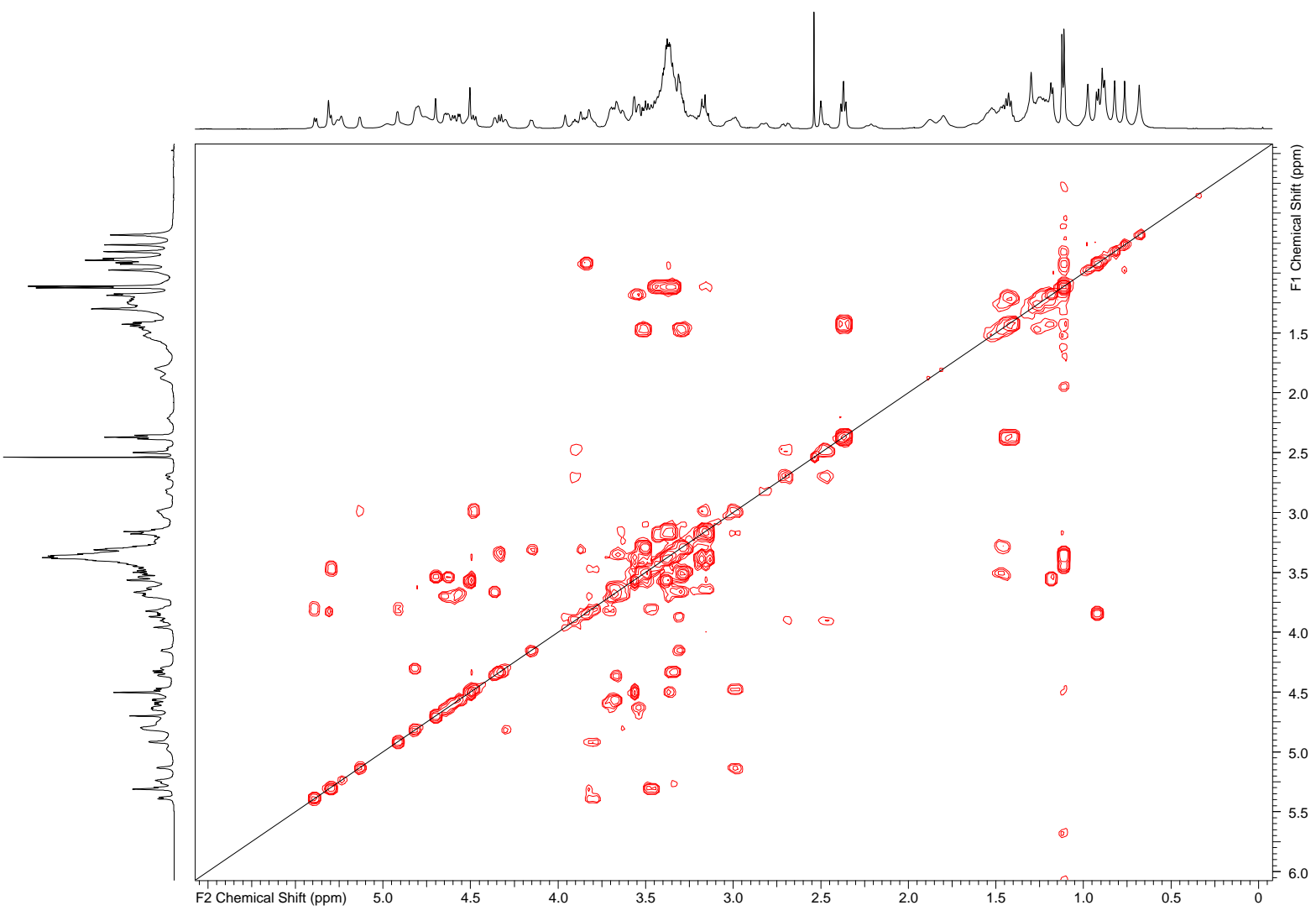

Figure S12. ${ }^{1} \mathrm{H}^{1}{ }^{\mathrm{H}} \mathrm{COSY}$ spectrum of compound $2\left(500 \mathrm{MHz}, \mathrm{DMSO}-\mathrm{d}_{6}\right)$.

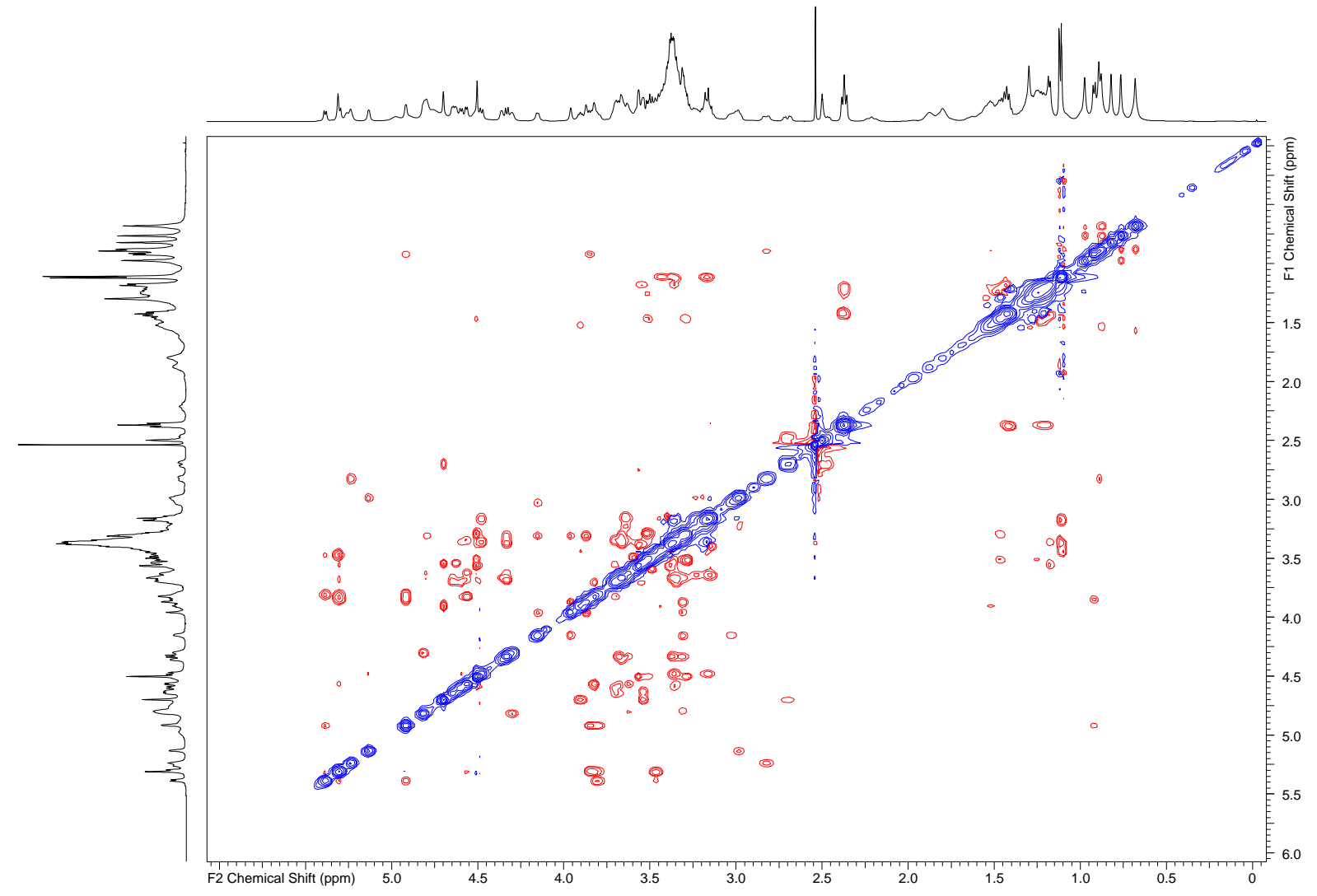

Figure S13. ${ }^{1} \mathrm{H}-{ }^{1} \mathrm{H}$ ROESY spectrum of compound 2 (500 MHz, DMSO-d 6 ). 


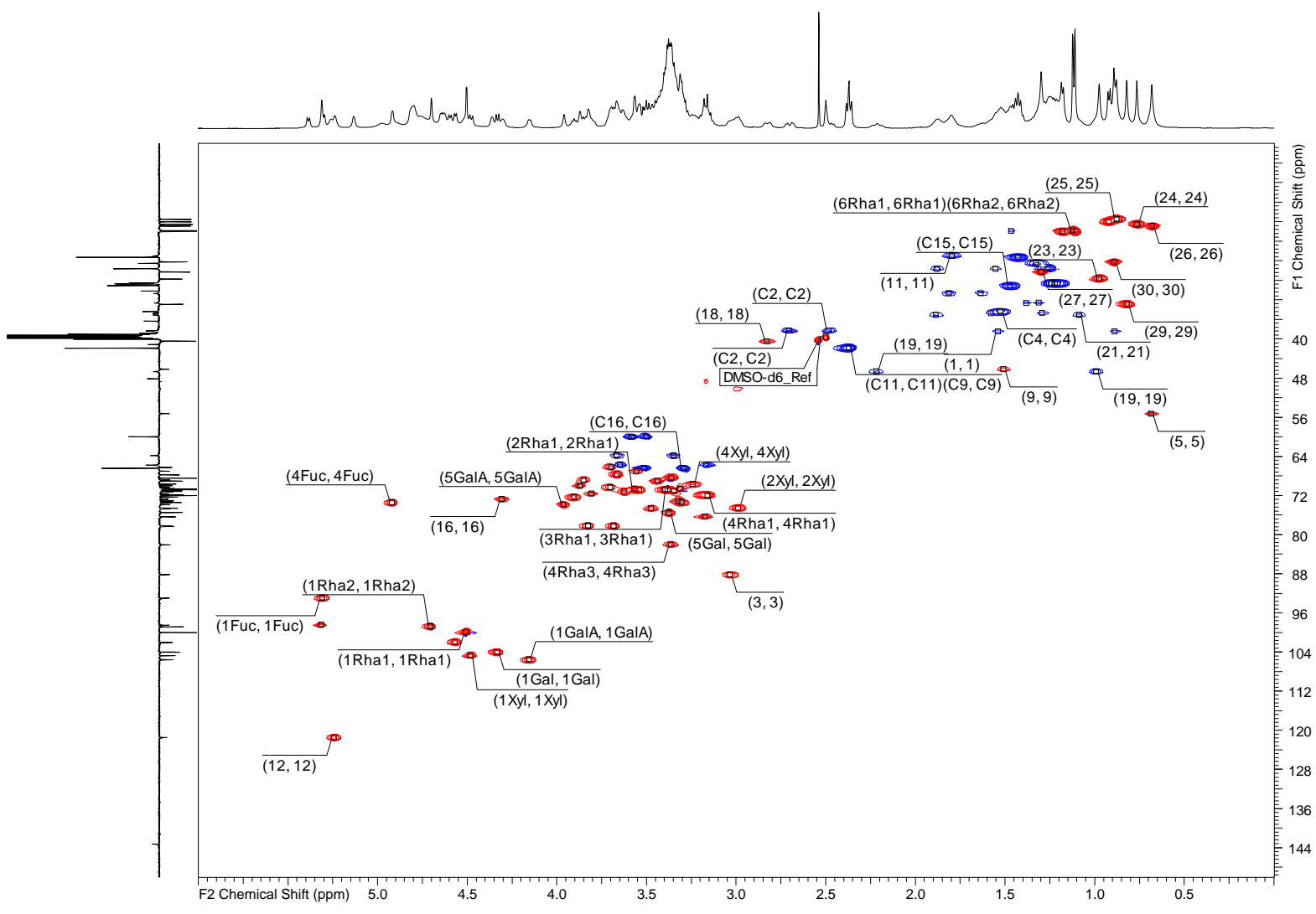

Figure S14. HSQC-DEPT spectrum of compound 2 (500 MHz, DMSO-d $)$.

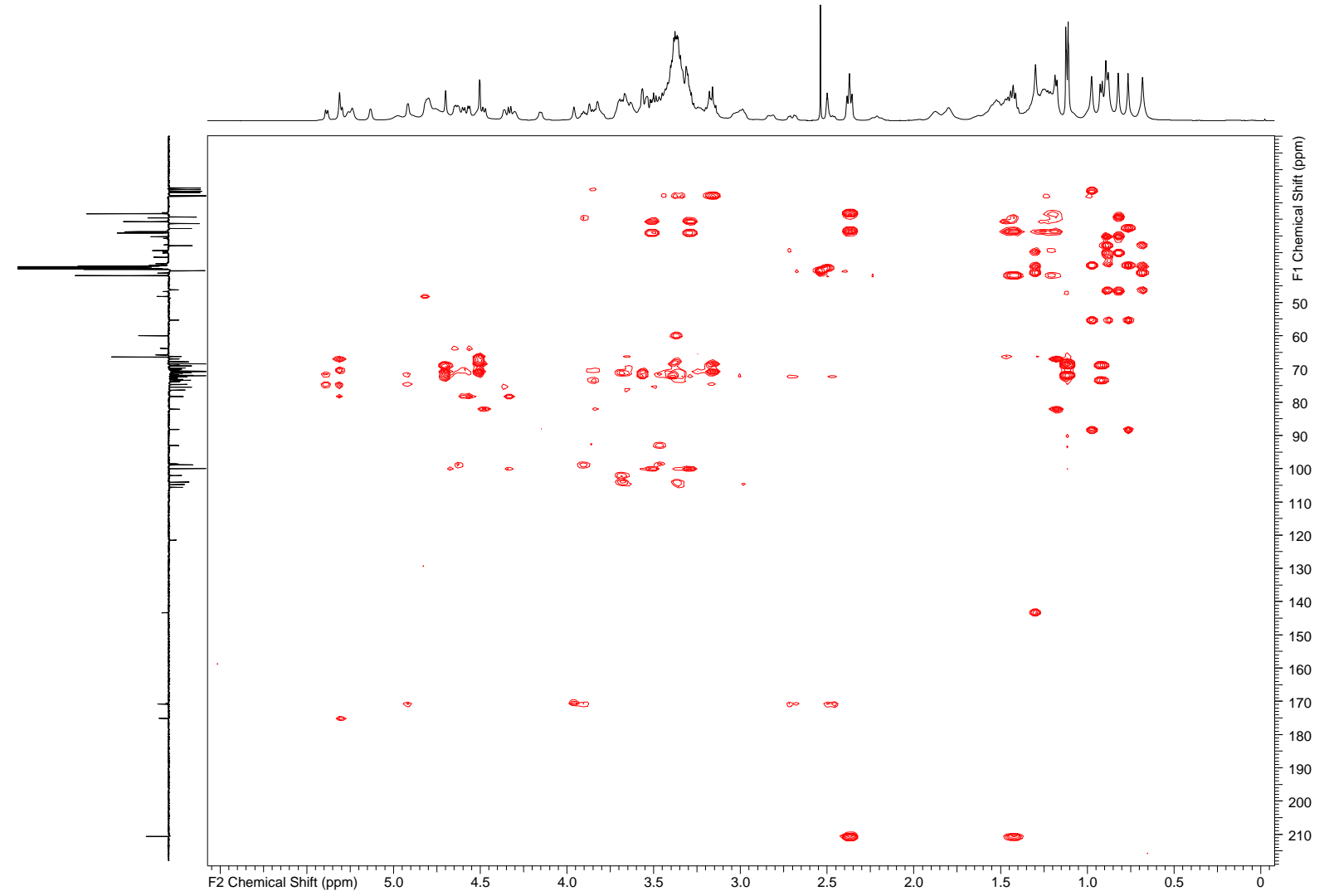

Figure S15. HMBC spectrum of compound 2 (500 MHz, DMSO-d6). 


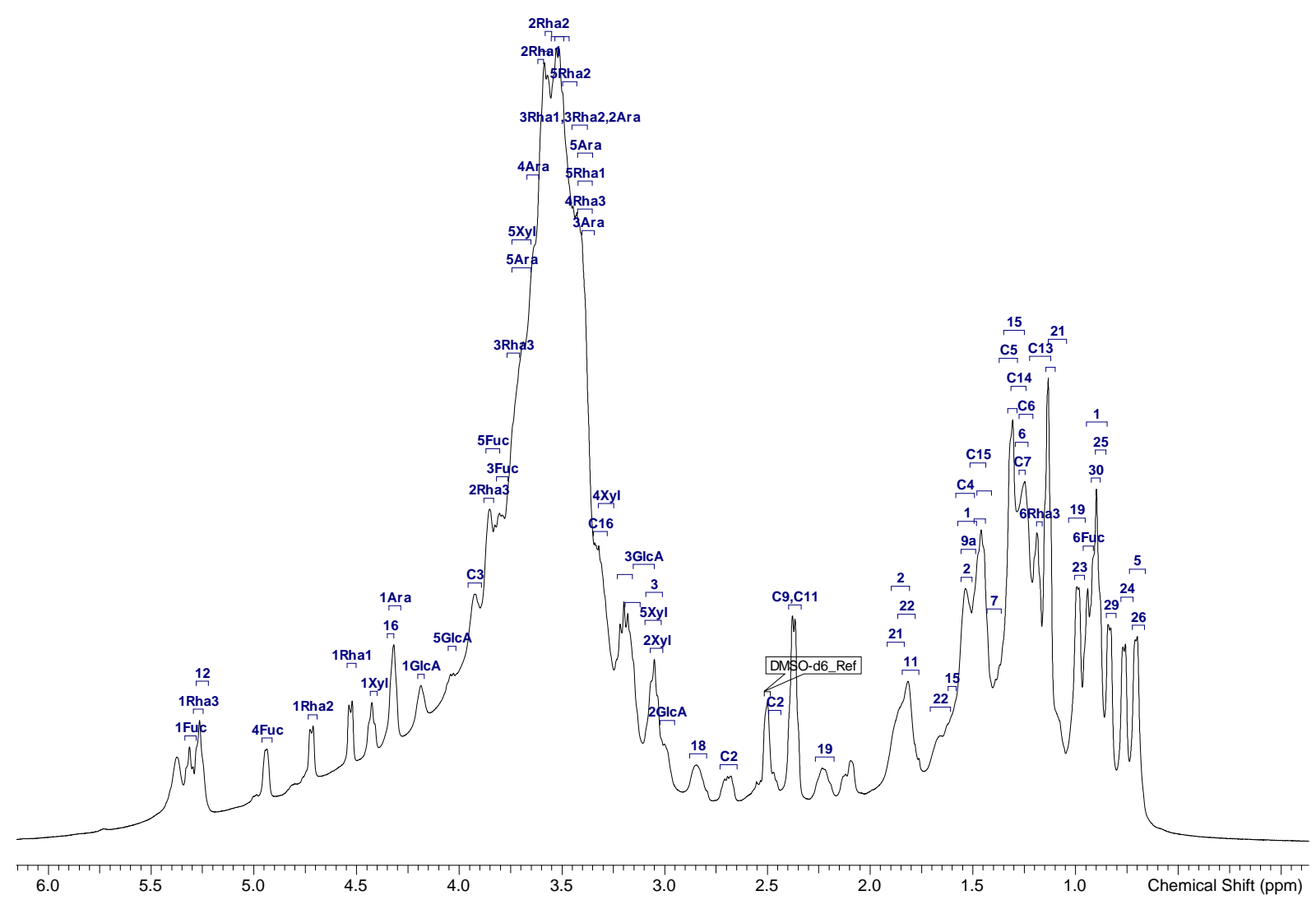

Figure S16. ${ }^{1} \mathrm{H}-\mathrm{NMR}$ spectrum of compound 3 (500 MHz, DMSO-d 6 ).

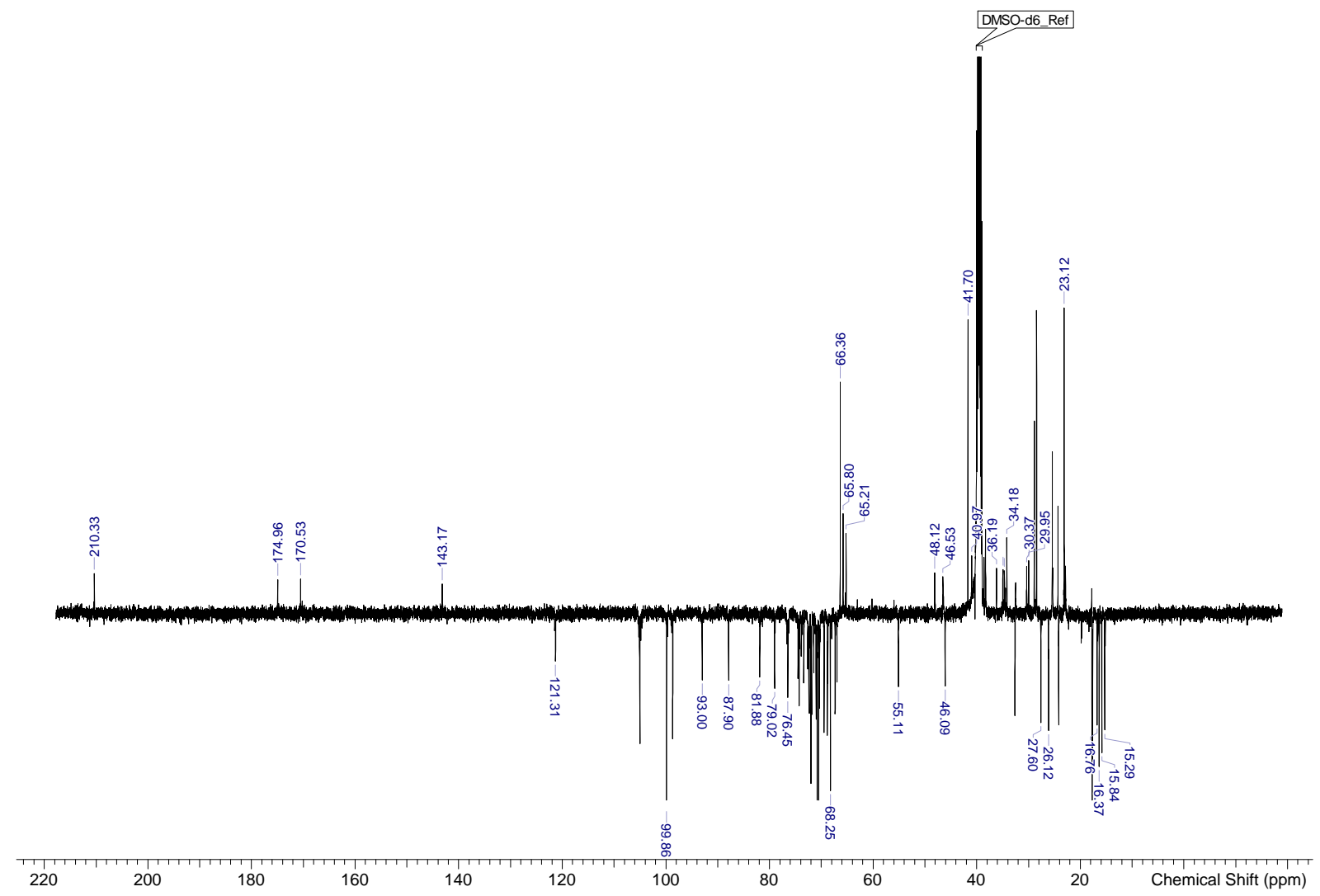

Figure S17. ${ }^{13} \mathrm{C}$-DEPTq spectrum of compound 3 (126 MHz, DMSO-d $)$. 


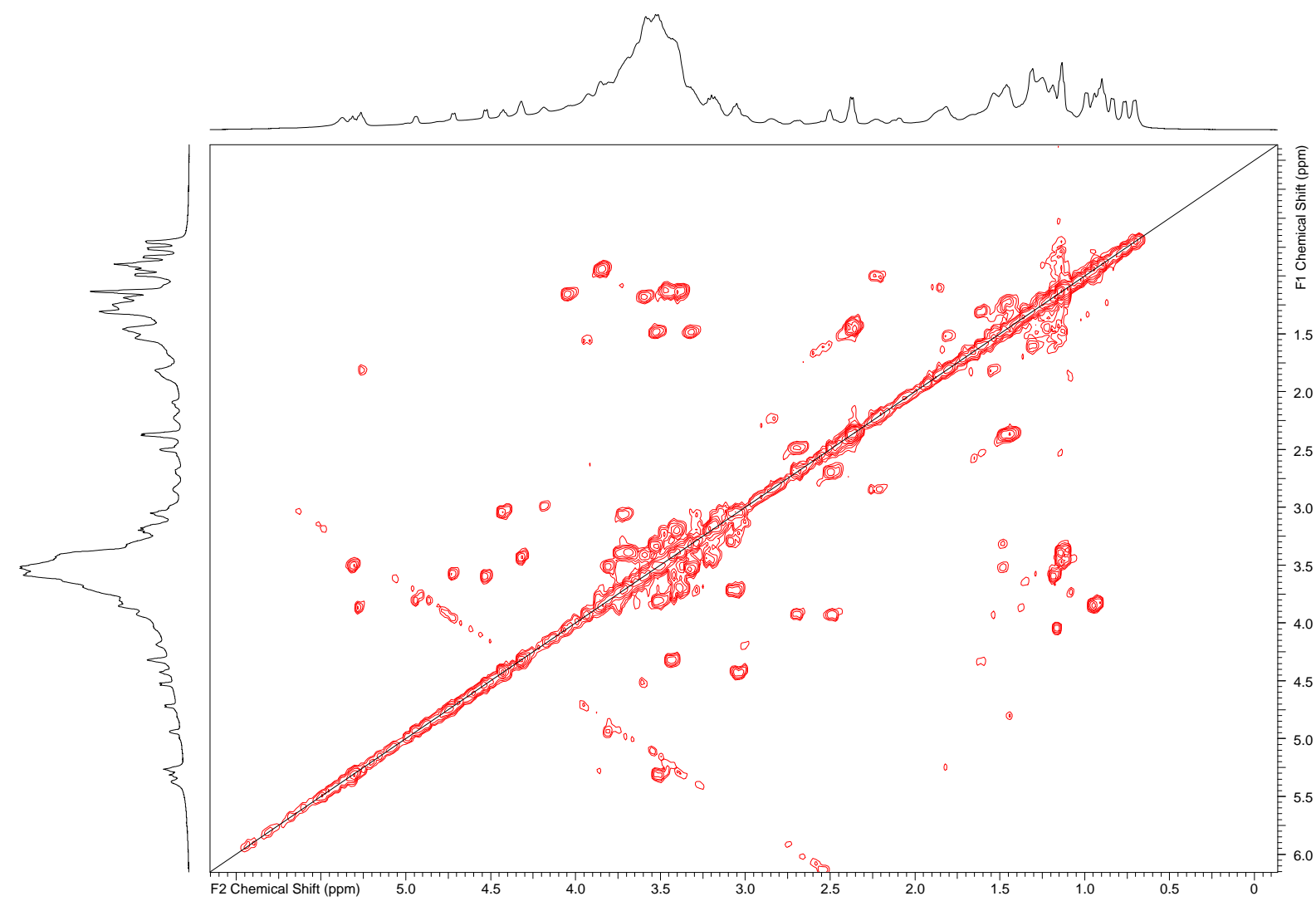

Figure S18. ${ }^{1} \mathrm{H}-{ }^{1} \mathrm{H}$ COSY spectrum of compound 3 (500 MHz, DMSO-d 6 ).

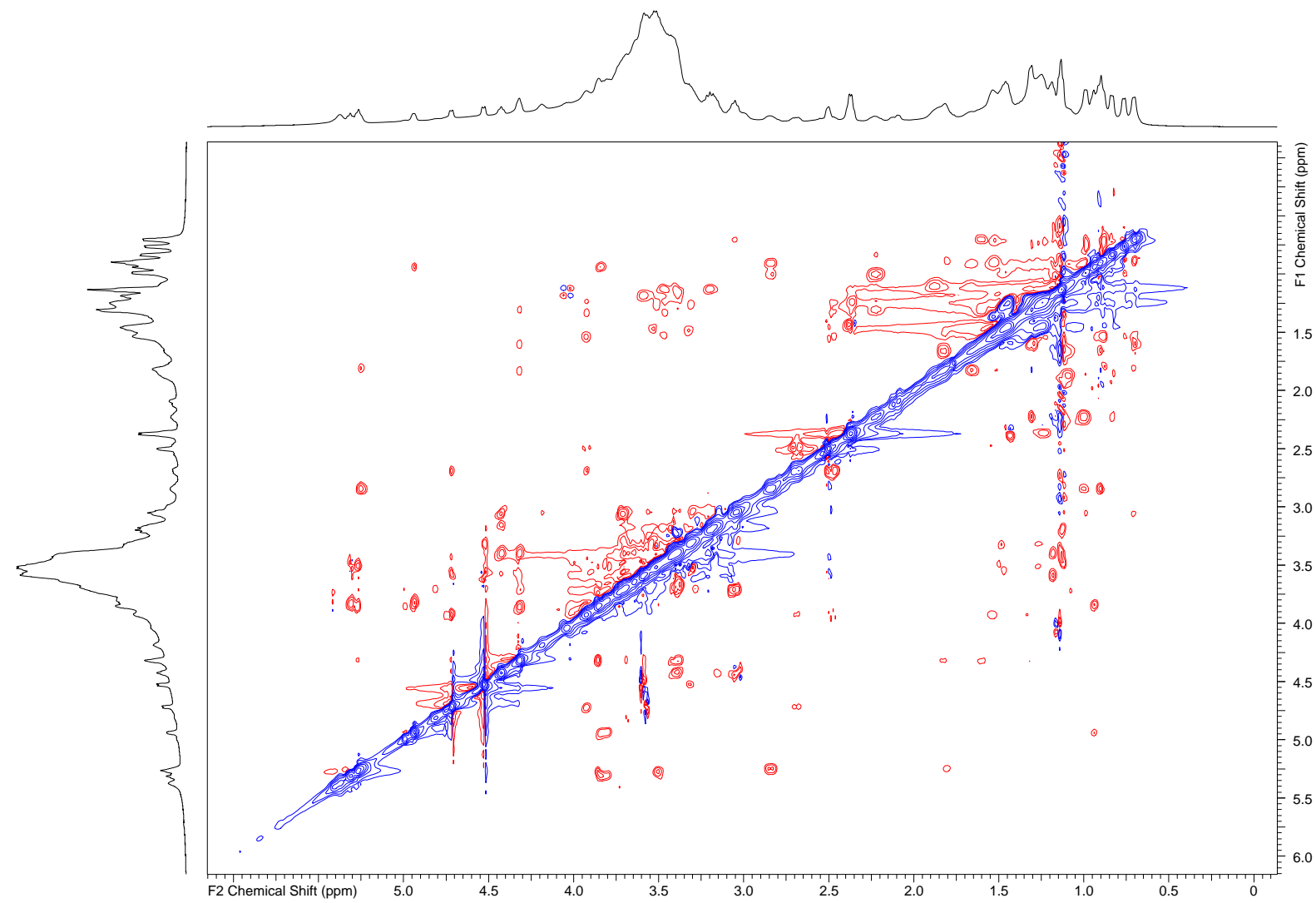

Figure S19. ${ }^{1} \mathrm{H}-{ }^{1} \mathrm{H}$ ROESY spectrum of compound 3 (500 MHz, DMSO-d6). 


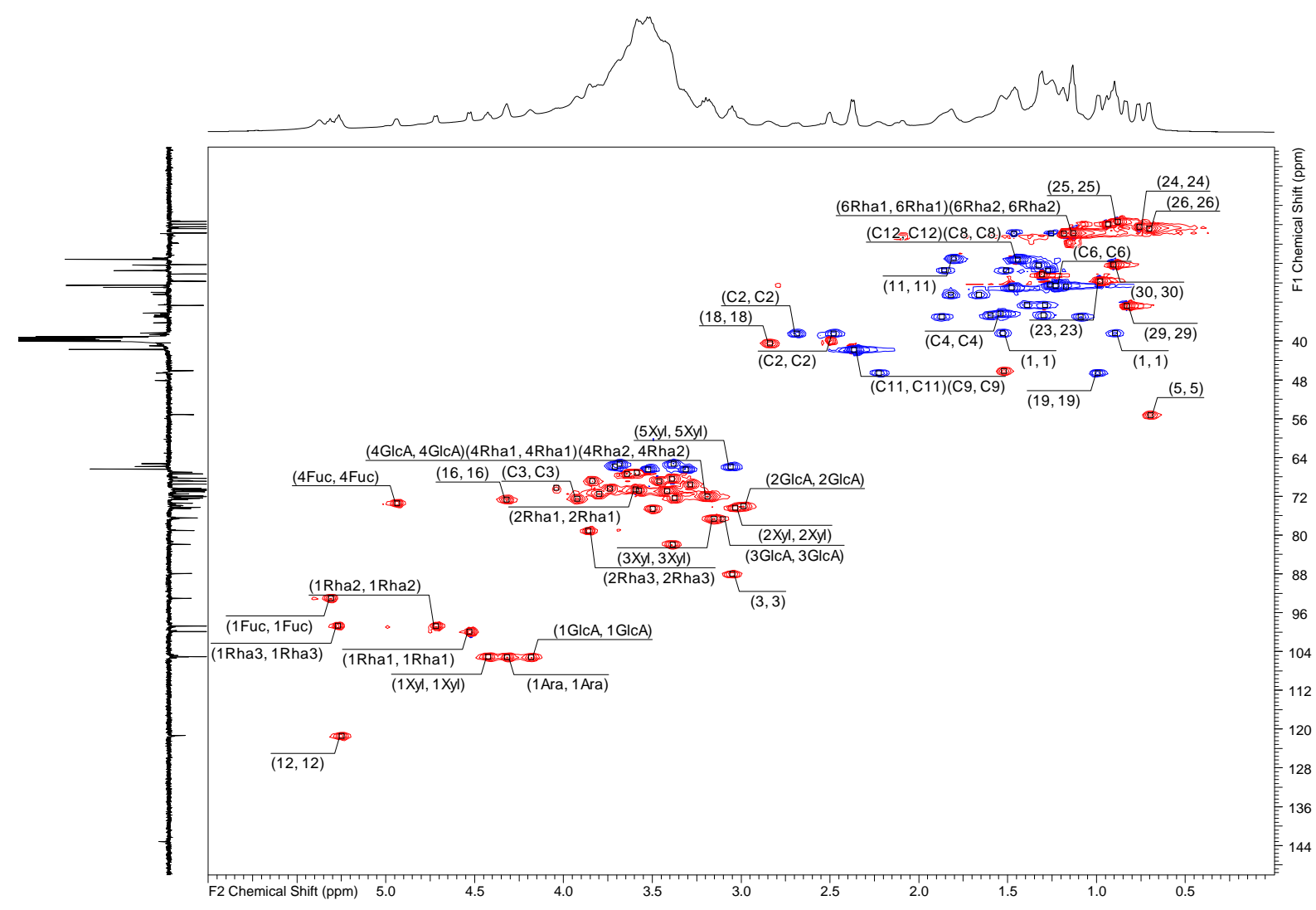

Figure S20. HSQC-DEPT spectrum of compound 3 (500 MHz, DMSO-d6).

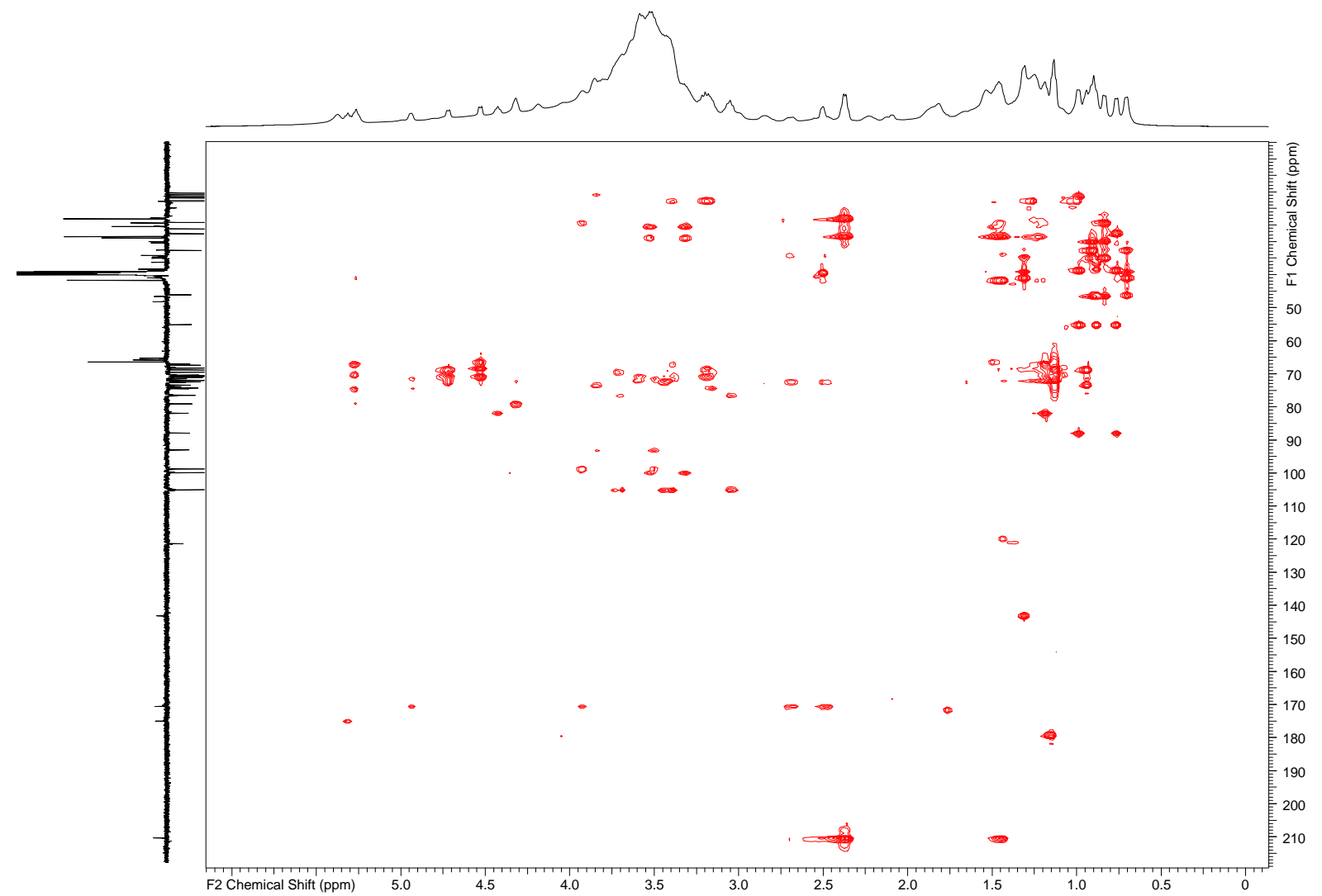

Figure S21. HMBC spectrum of compound 3 (500 MHz, DMSO-d $)$. 


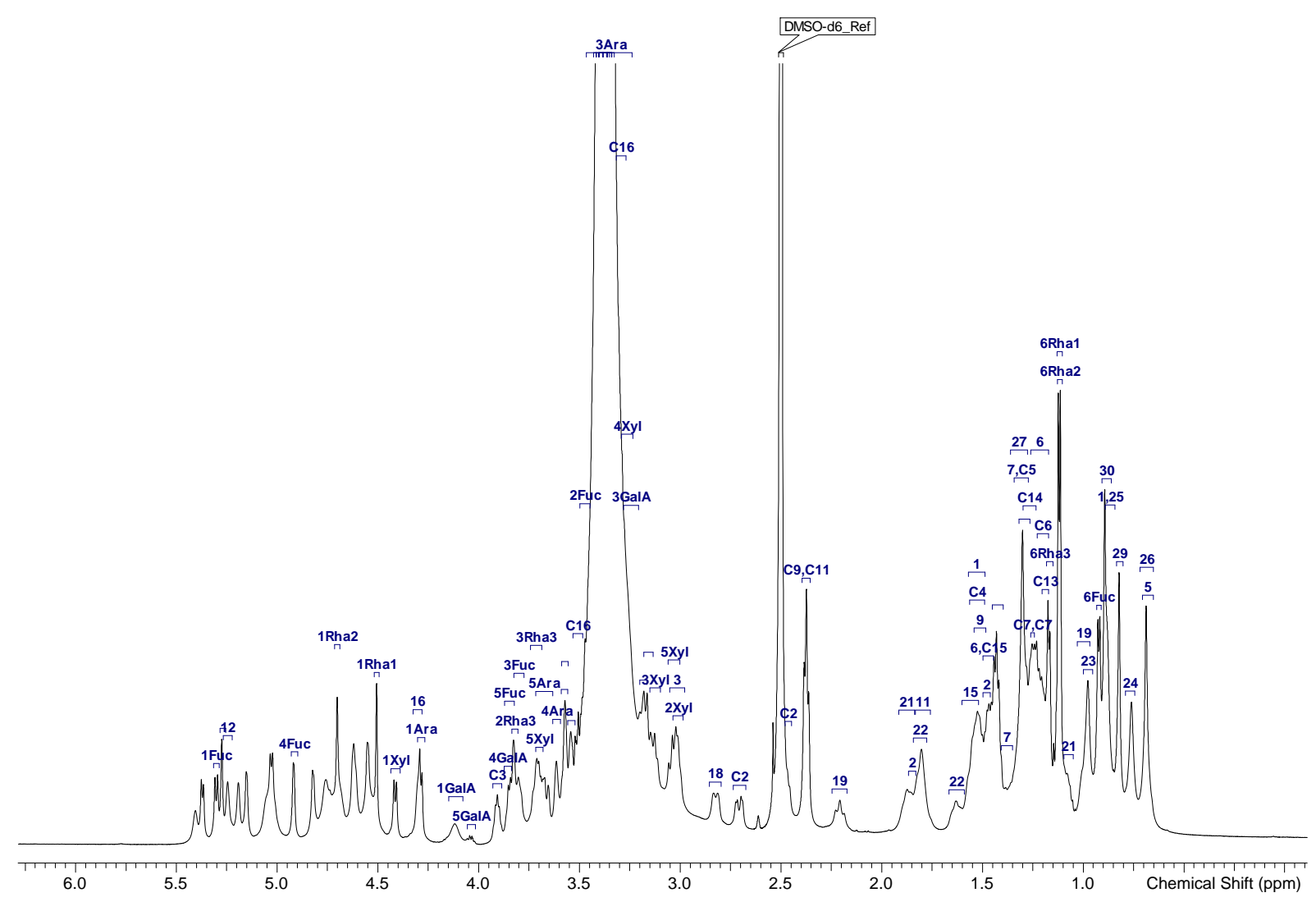

Figure S22. ${ }^{1} \mathrm{H}-\mathrm{NMR}$ spectrum of compound 4 (600 MHz, DMSO-d 6 ).

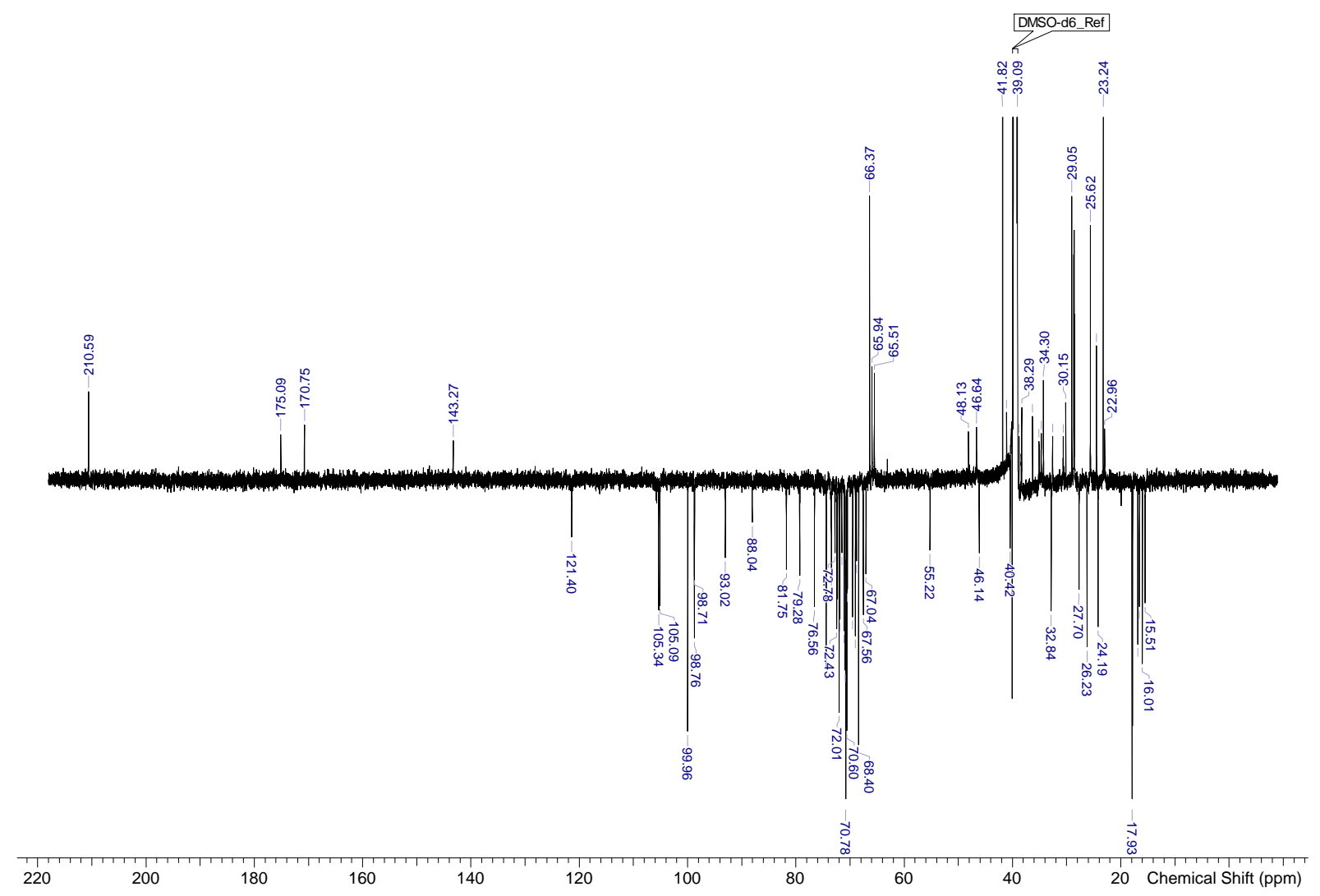

Figure S23. ${ }^{13} \mathrm{C}-\mathrm{DEPT}$ spectrum of compound 4 (151 MHz, DMSO-d 6 ). 


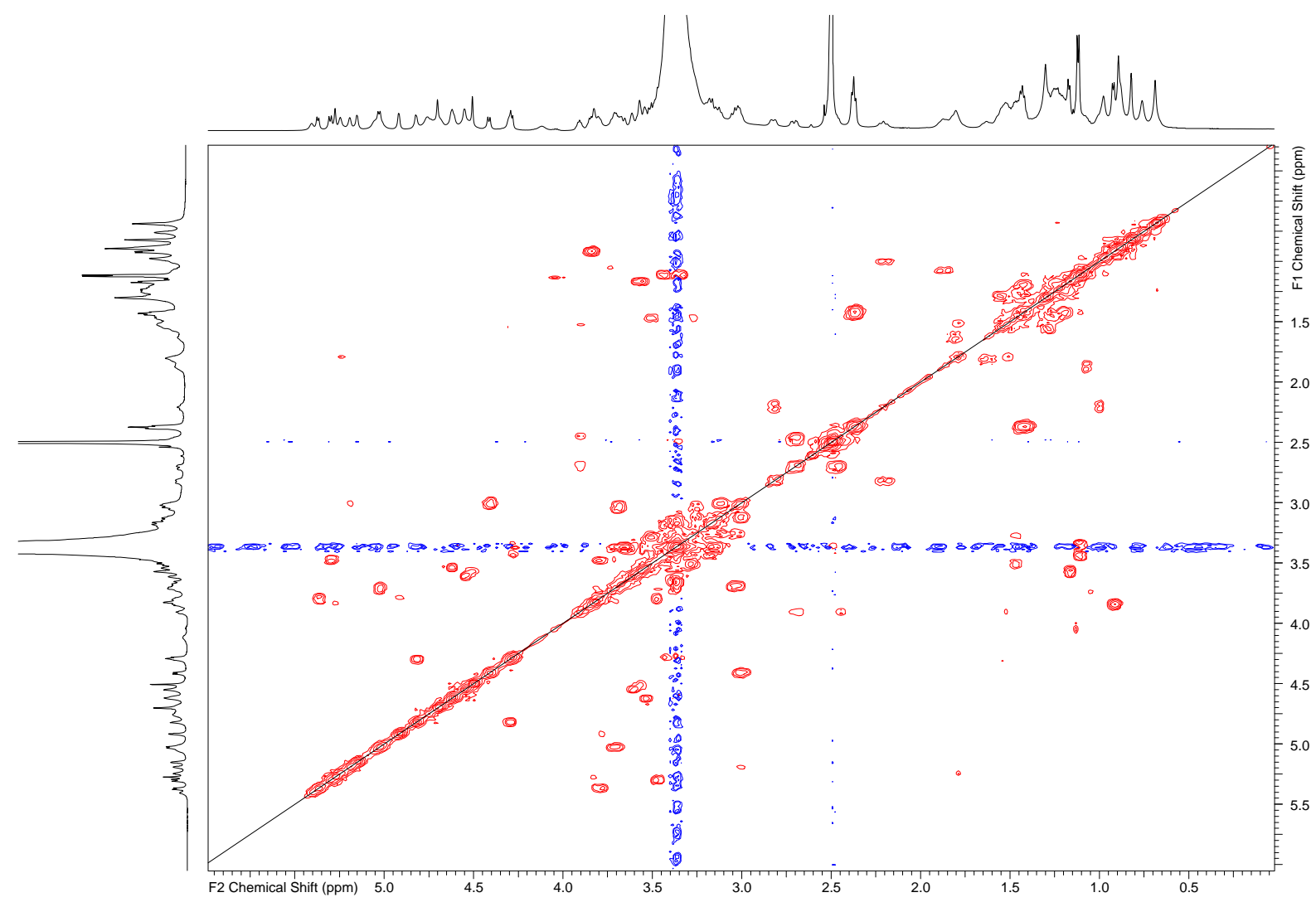

Figure S24. ${ }^{1} \mathrm{H}-{ }^{1} \mathrm{H}$ COSY spectrum of compound 4 (600 MHz, DMSO-d6).

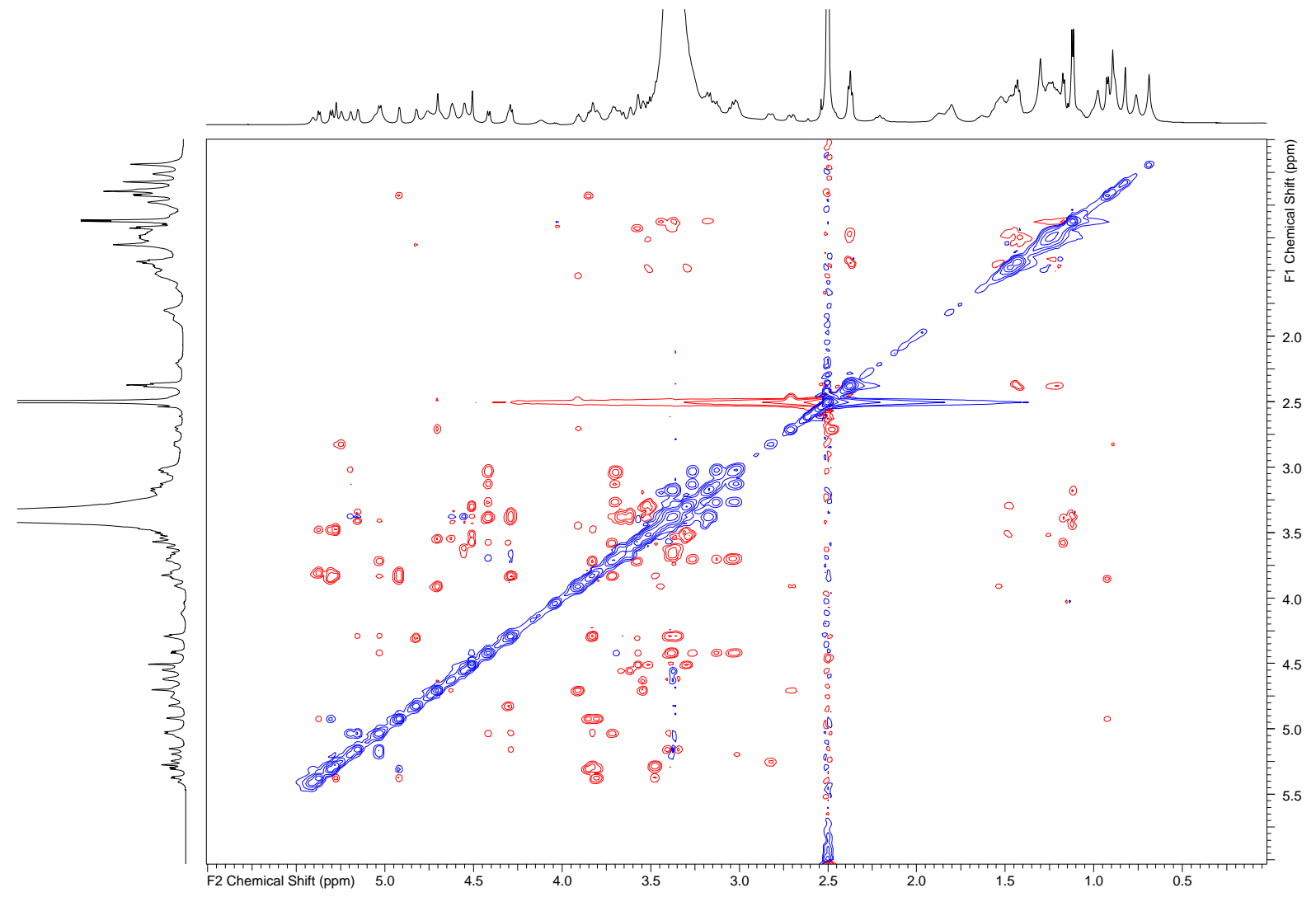

Figure S25. ${ }^{1} \mathrm{H}-{ }^{1} \mathrm{H}$ ROESY spectrum of compound 4 (600 MHz, DMSO-d 6 ). 


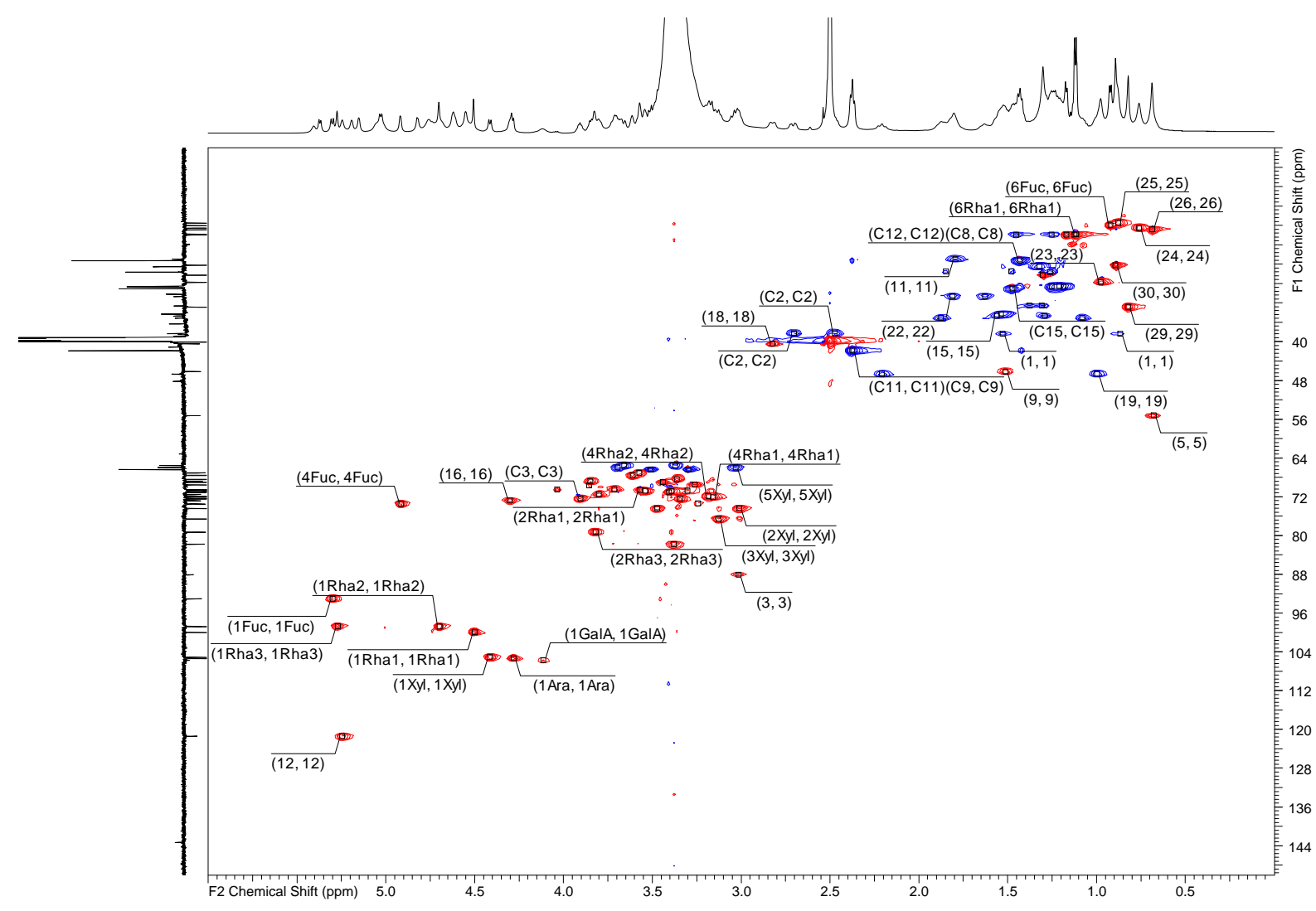

Figure S26. HSQC-DEPT spectrum of compound 4 (600 MHz, DMSO-d6).

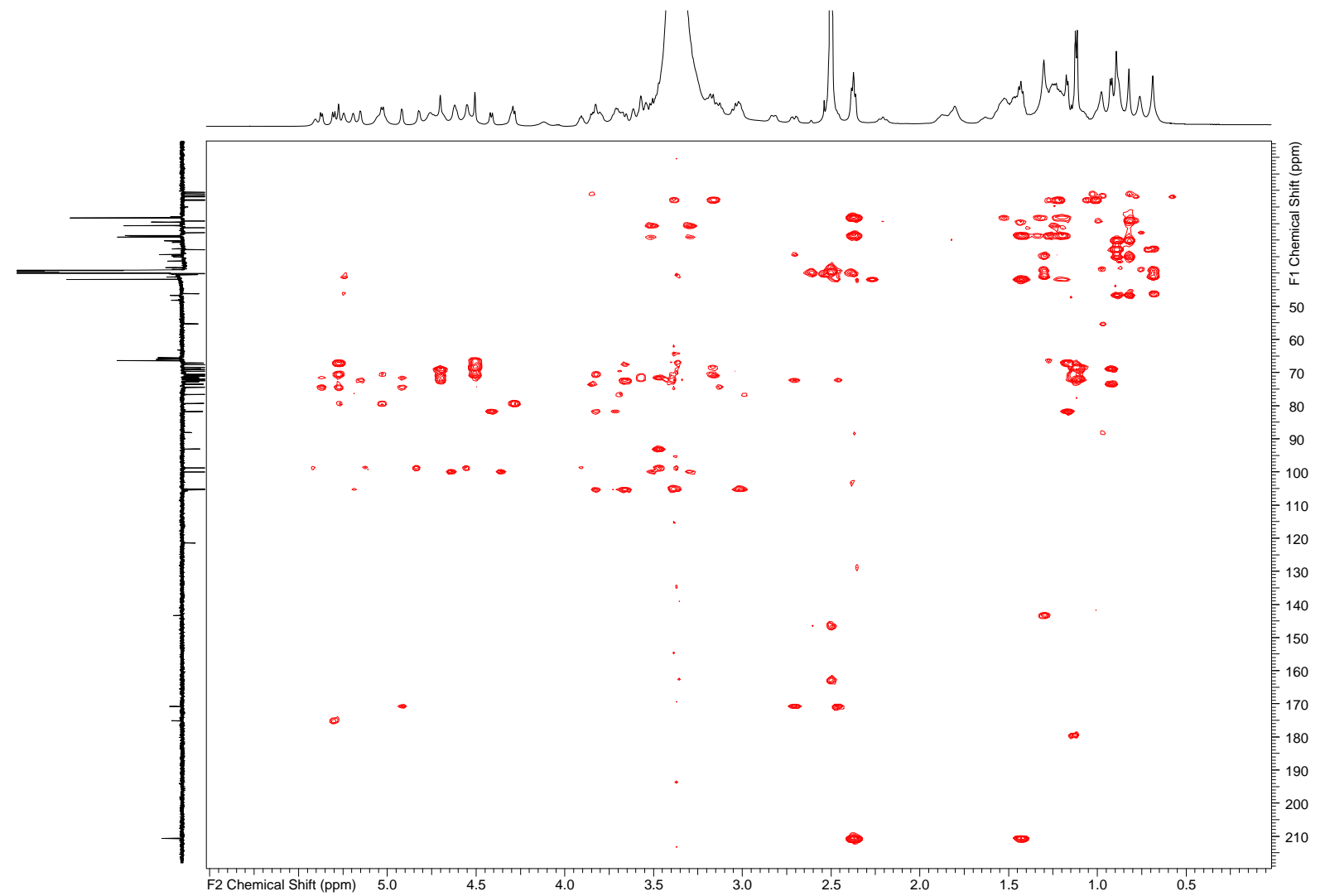

Figure S27. HMBC spectrum of compound 4 (600 MHz, DMSO-d $)$. 


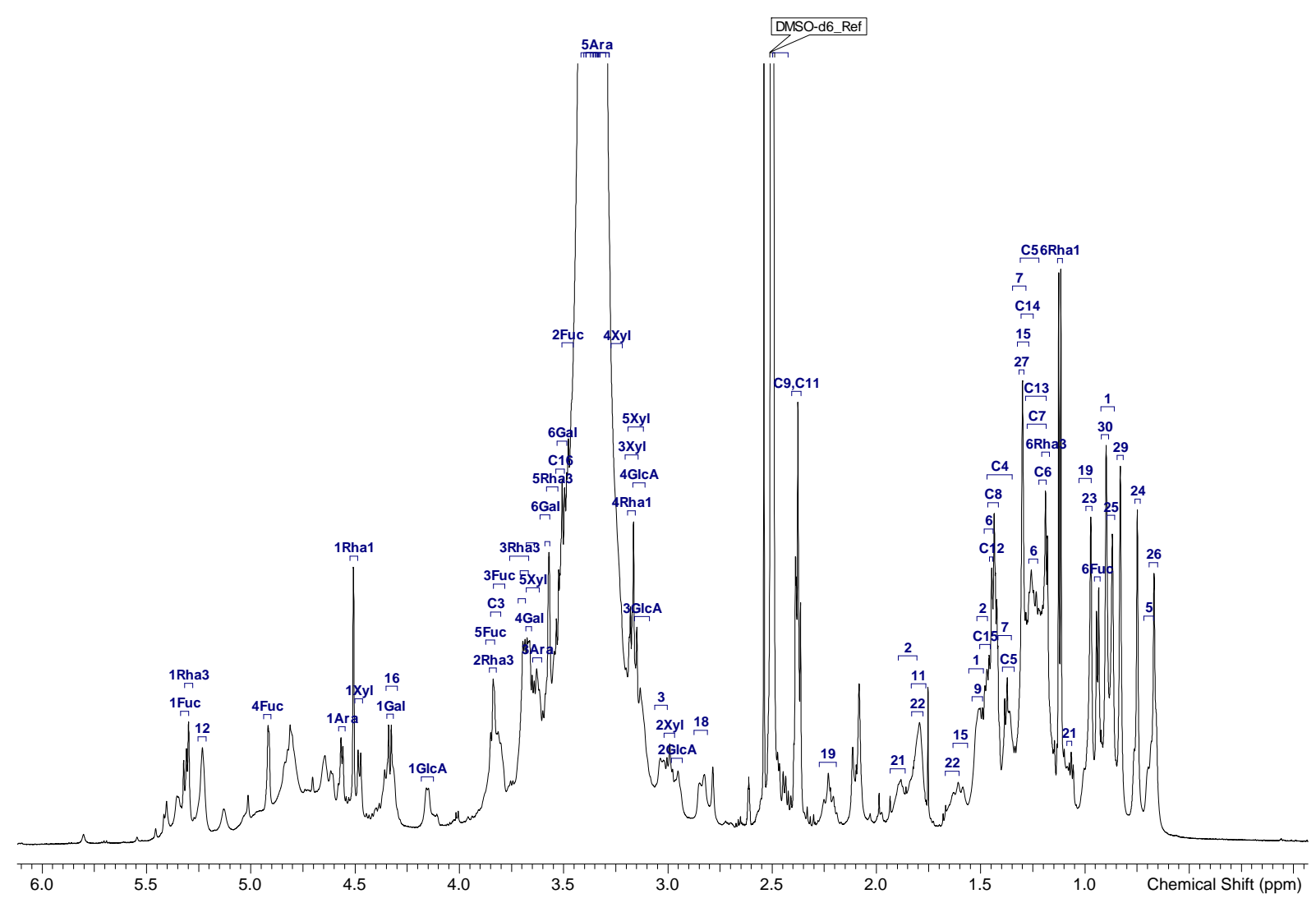

Figure S28. ${ }^{1} \mathrm{H}-\mathrm{NMR}$ spectrum of compound 5 (600 MHz, DMSO-d 6 ).

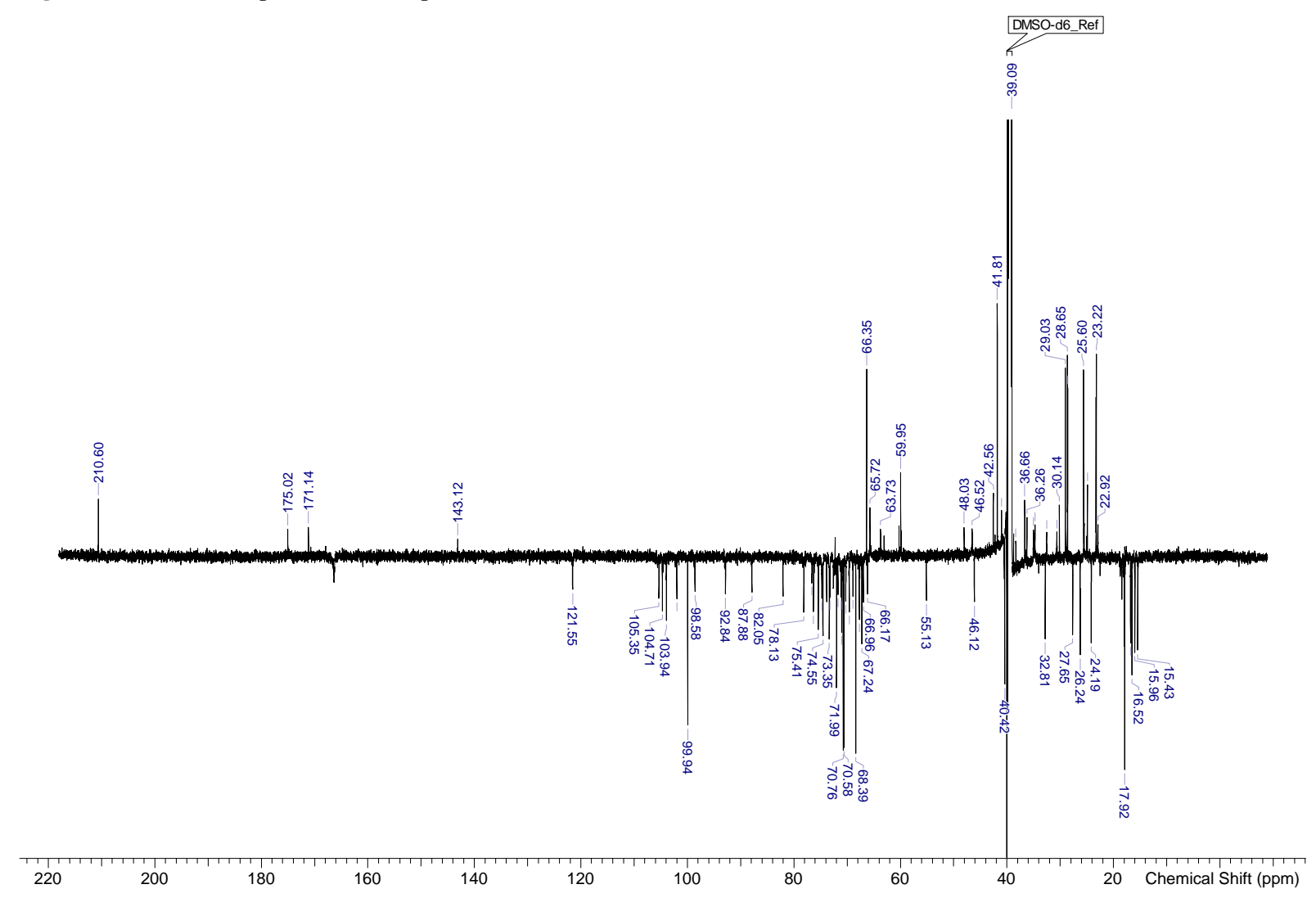

Figure S29. ${ }^{13} \mathrm{C}-\mathrm{DEPTq}$ spectrum of compound 5 (151 MHz, DMSO-d 6 ). 


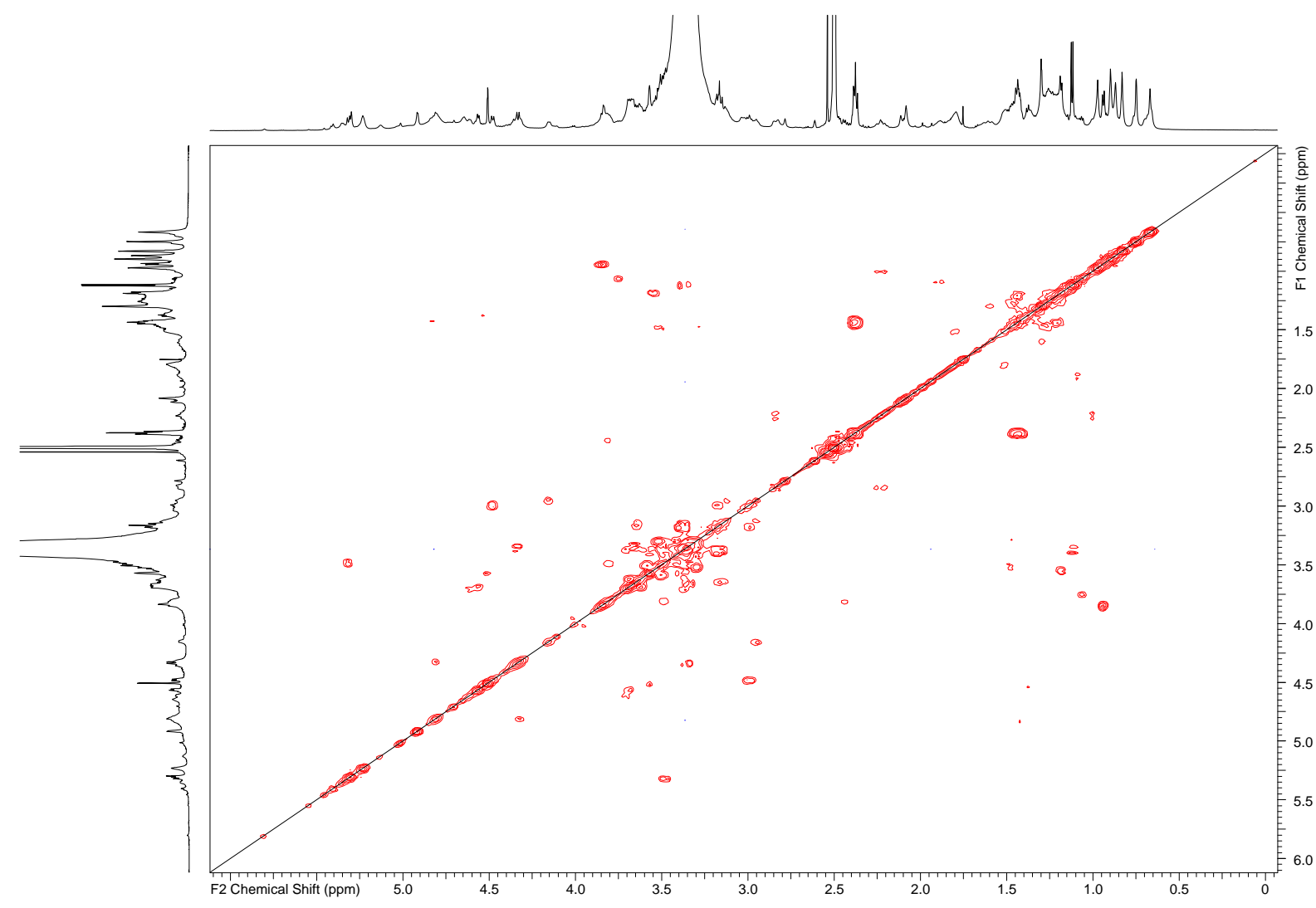

Figure S30. ${ }^{1} \mathrm{H}-{ }^{1} \mathrm{H}$ COSY spectrum of compound 5 (600 MHz, DMSO-d 6 ).

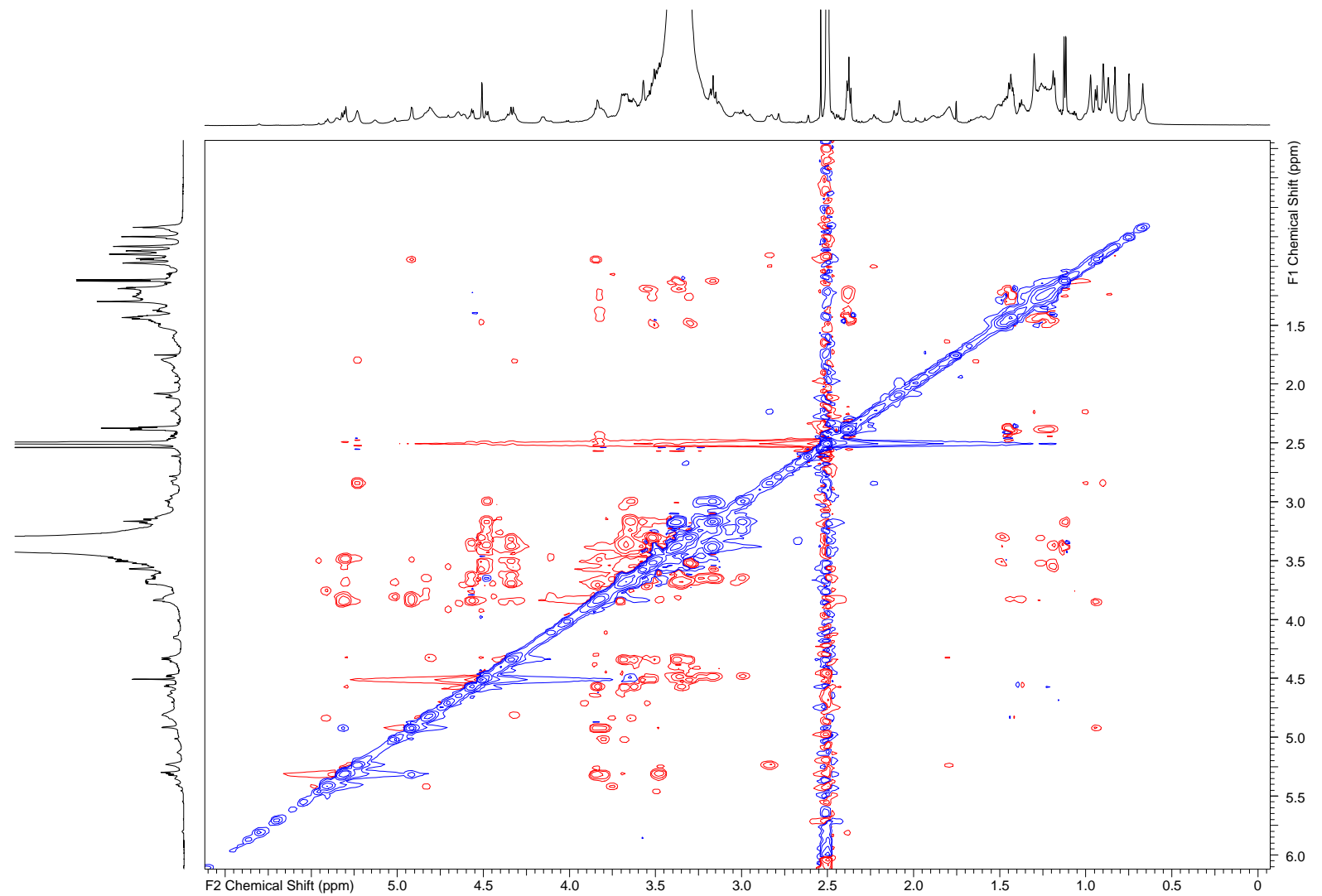

Figure S31. ${ }^{1} \mathrm{H}-{ }^{1} \mathrm{H}$ ROESY spectrum of compound 5 (600 MHz, DMSO-d6). 


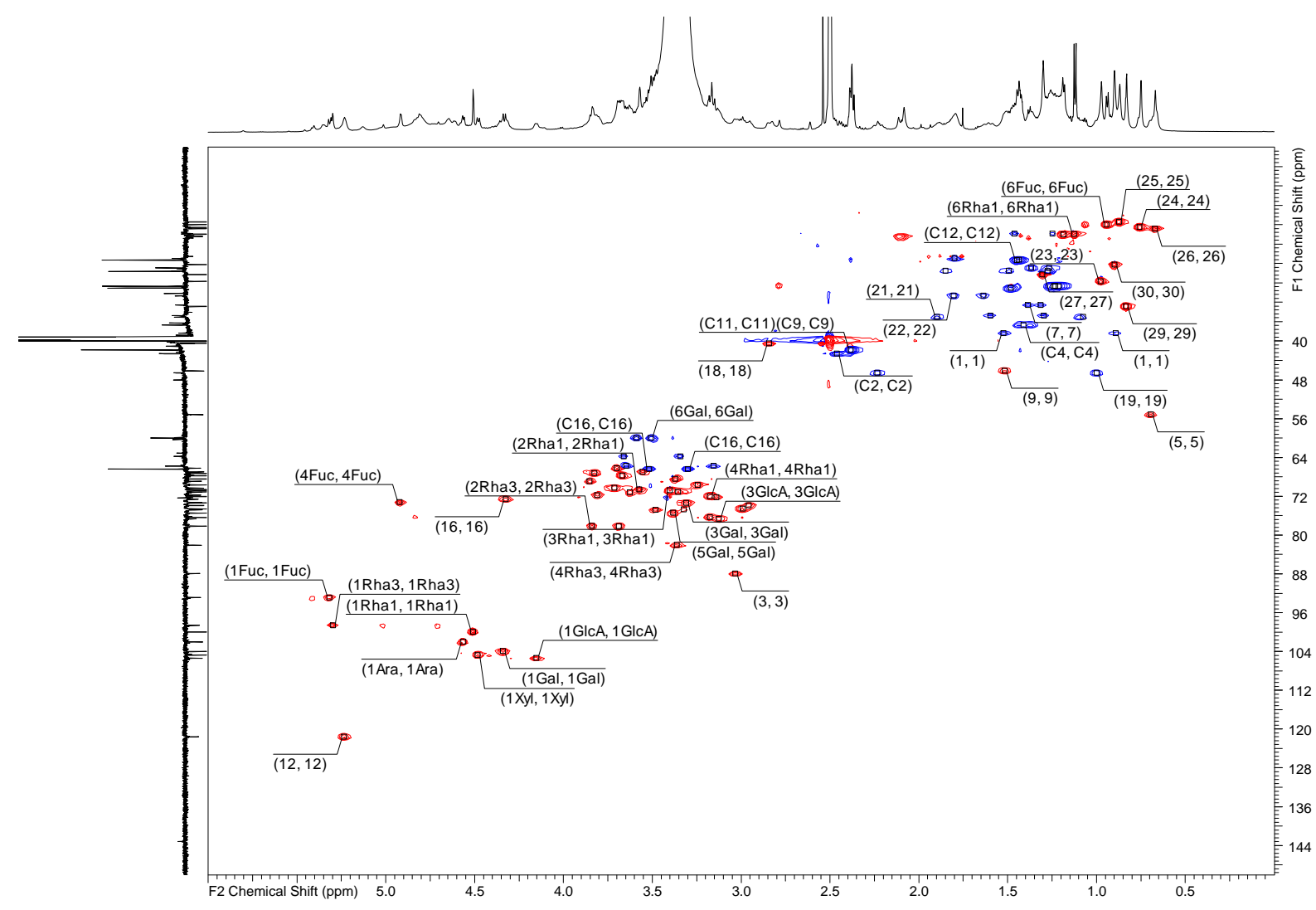

Figure S32. HSQC-DEPT spectrum of compound 5 (600 MHz, DMSO-d6).

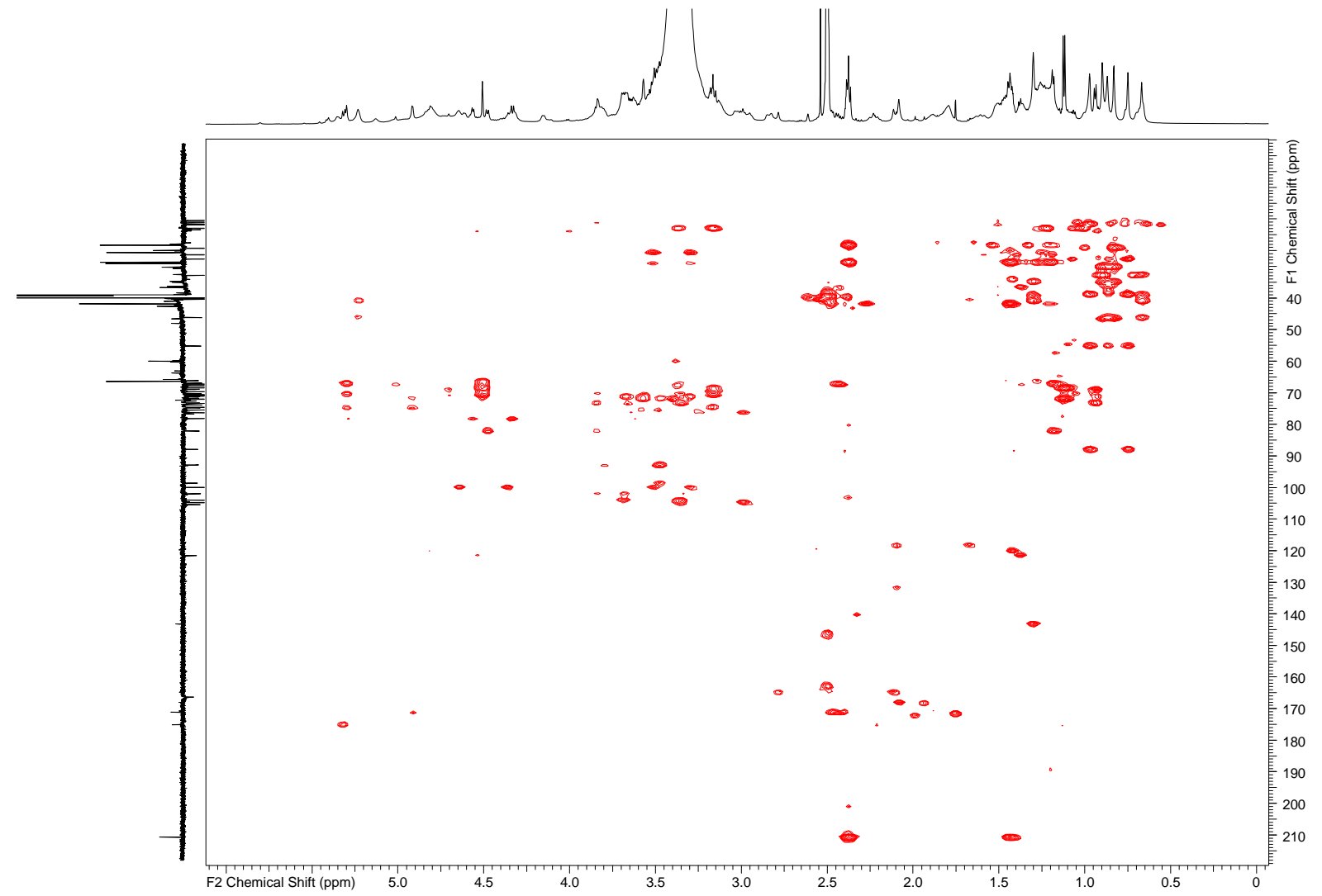

Figure S33. HMBC spectrum of compound 5 (600 MHz, DMSO-d $)$. 


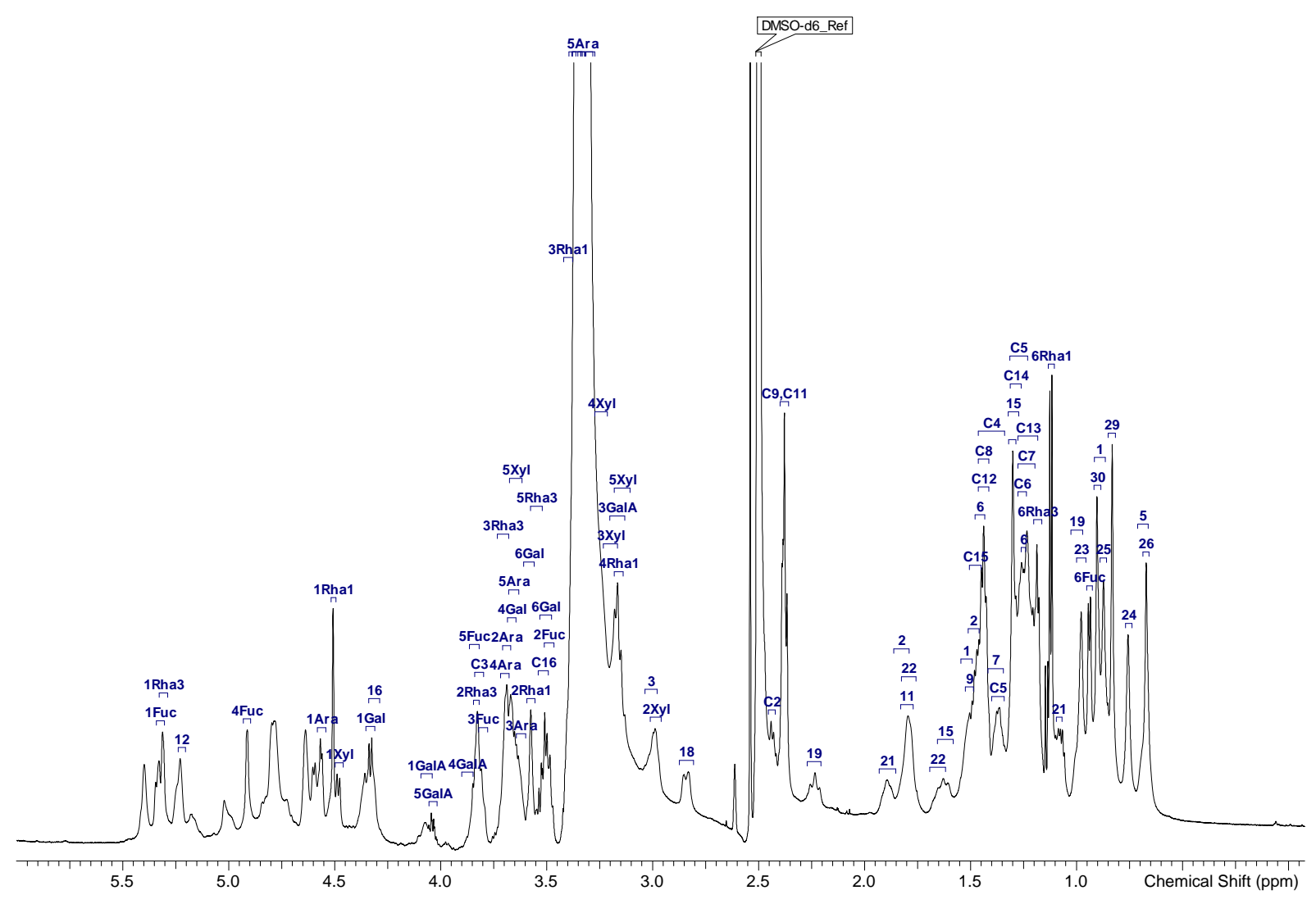

Figure S34. ${ }^{1} \mathrm{H}-\mathrm{NMR}$ spectrum of compound 6 (600 MHz, DMSO-d 6 ).

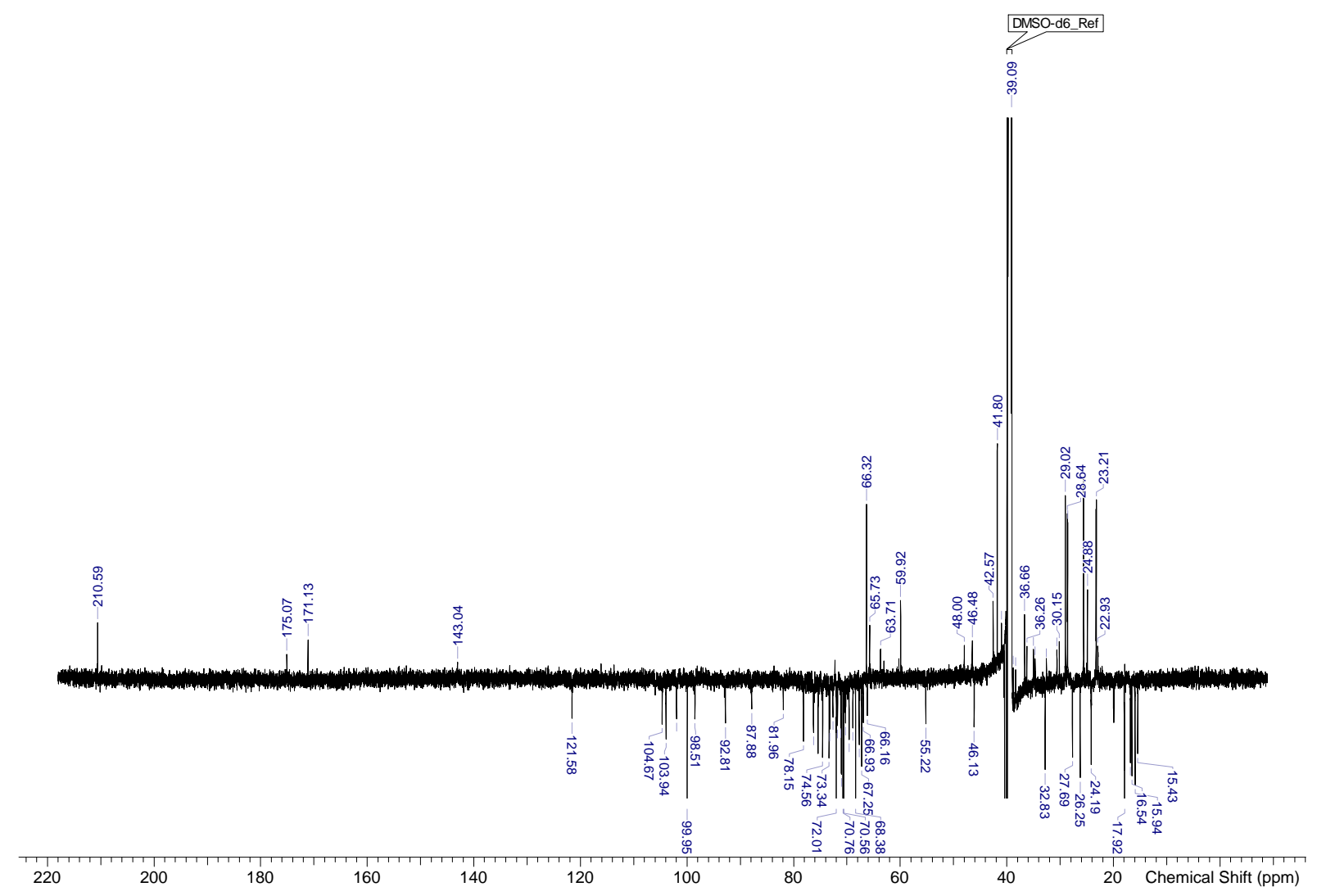

Figure S35. ${ }^{13} \mathrm{C}$-DEPTq spectrum of compound 6 (151 MHz, DMSO-d $)$. 


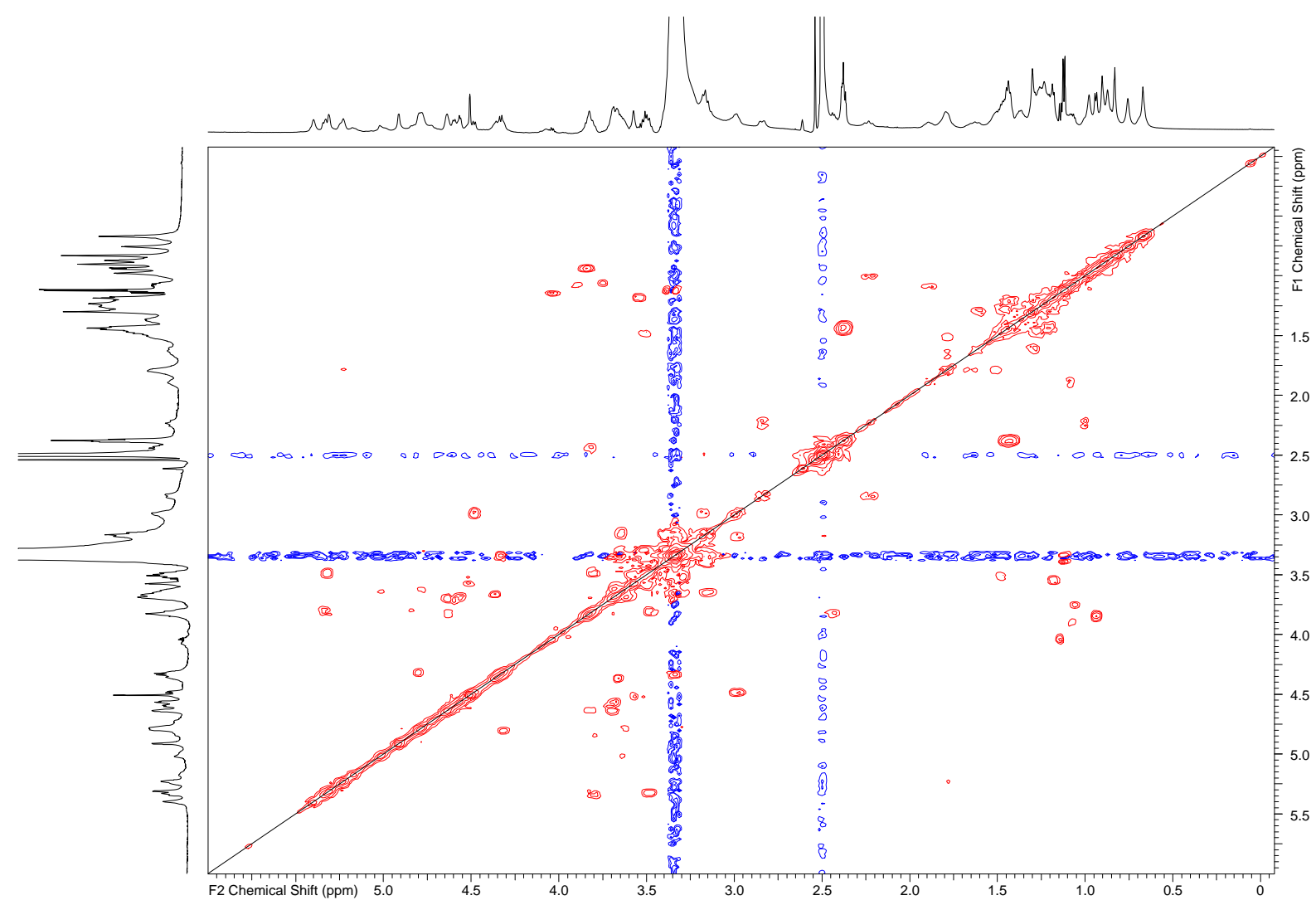

Figure S36. ${ }^{1} \mathrm{H}-{ }^{1} \mathrm{H}$ COSY spectrum of compound 6 (600 MHz, DMSO-d 6 ).

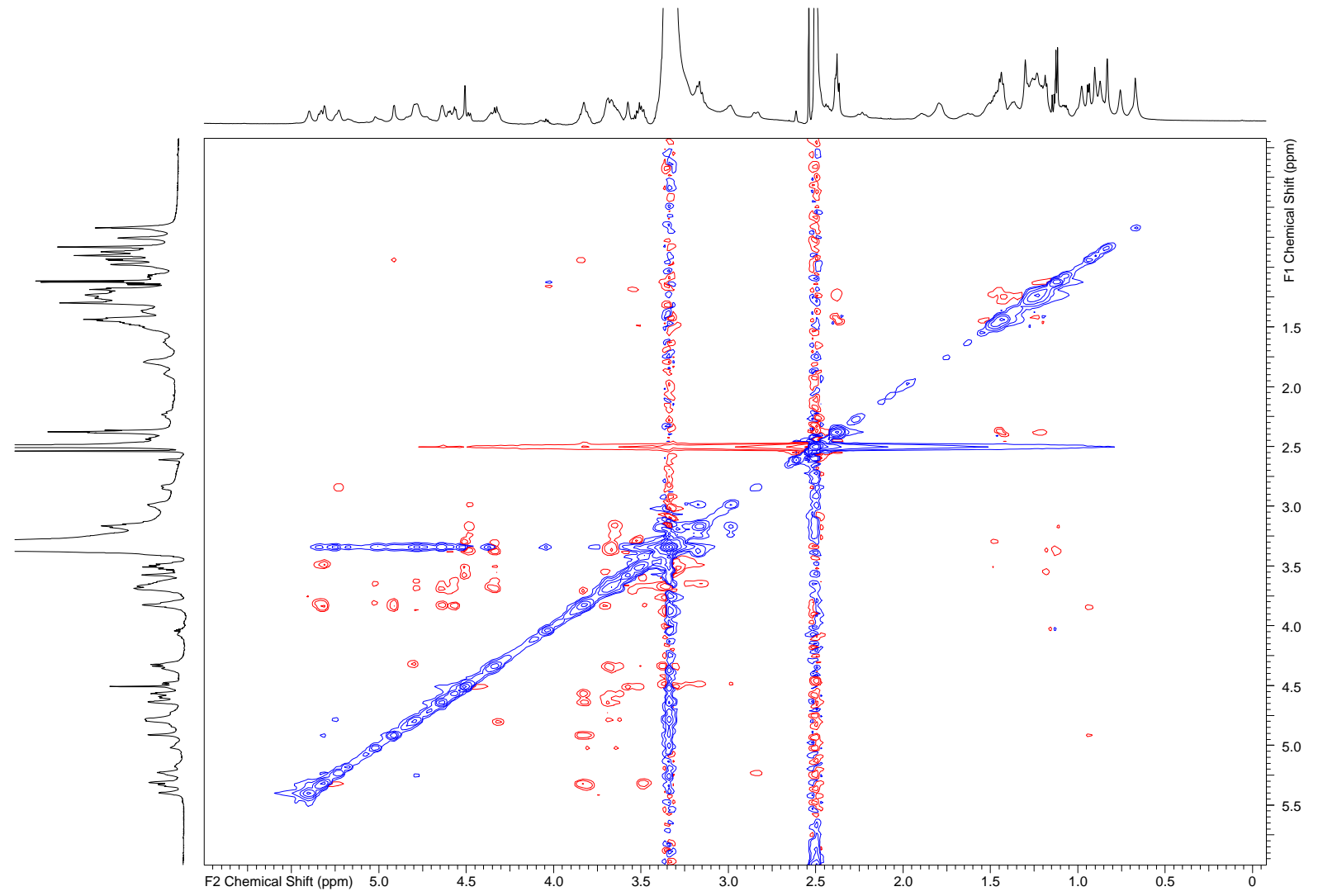

Figure S37. ${ }^{1} \mathrm{H}-{ }^{1} \mathrm{H}$ ROESY spectrum of compound 6 (600 MHz, DMSO-d6). 


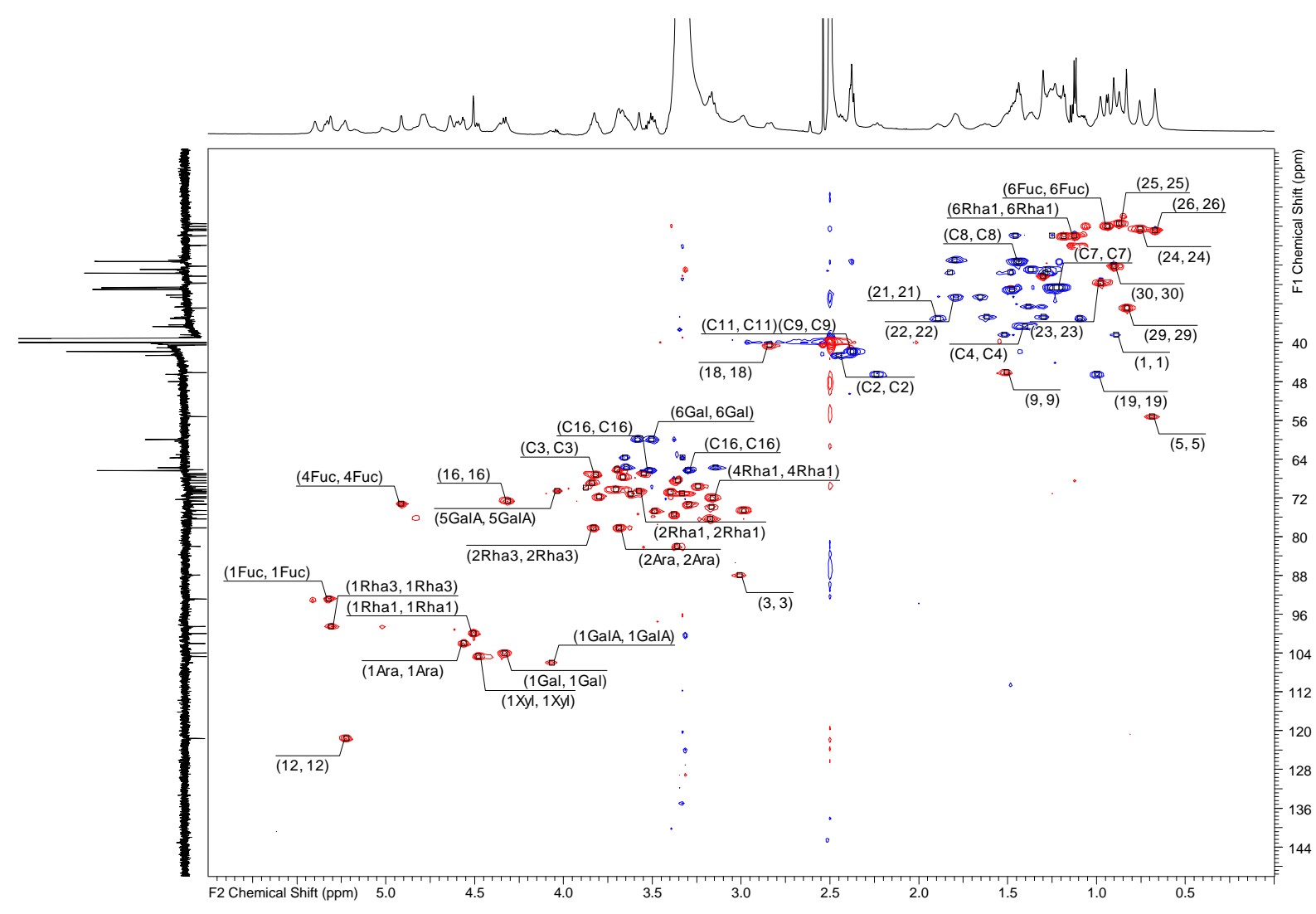

Figure S38. HSQC-DEPT spectrum of compound 6 (600 MHz, DMSO-d6).

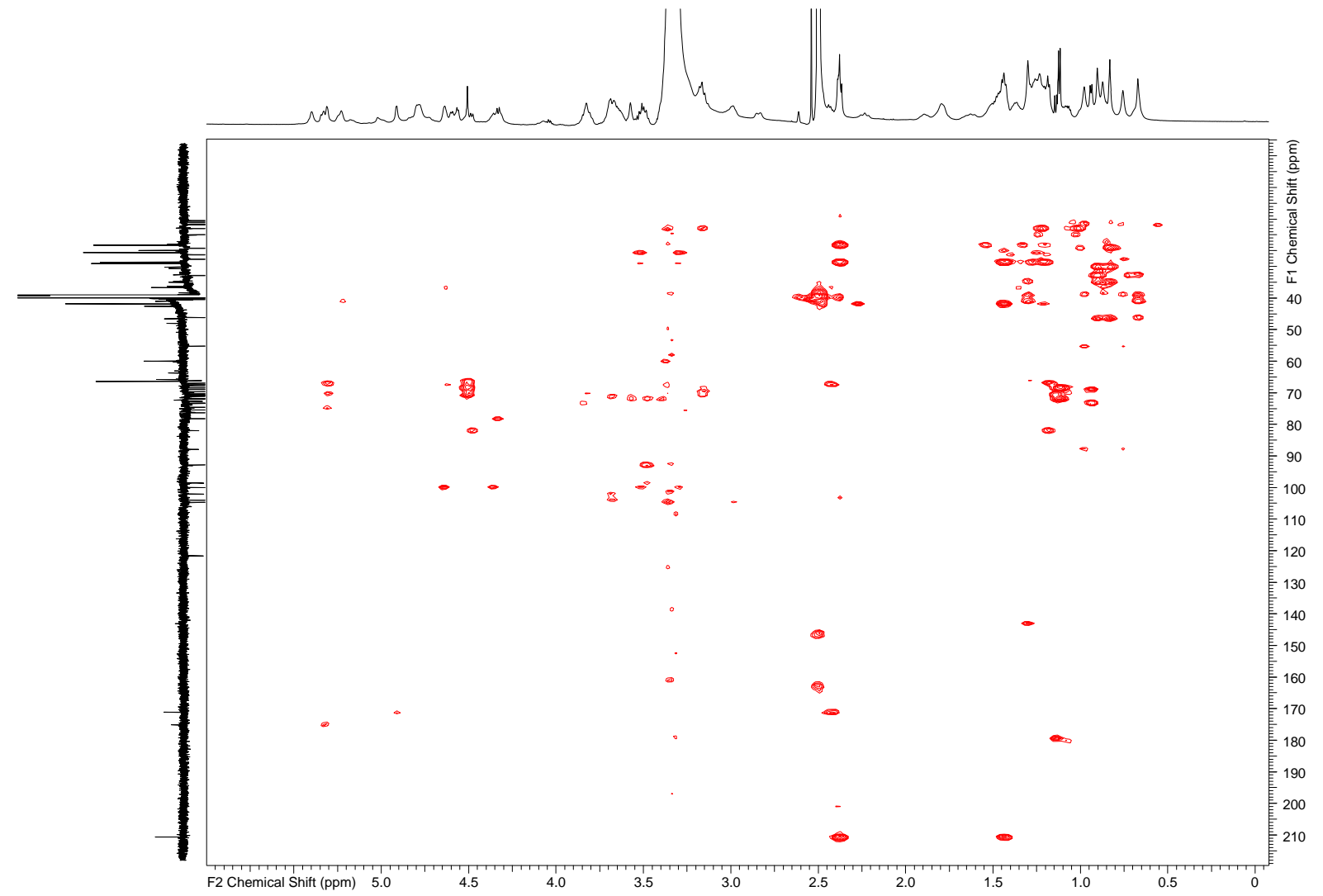

Figure S39. HMBC spectrum of compound 6 (600 MHz, DMSO-d $)$. 


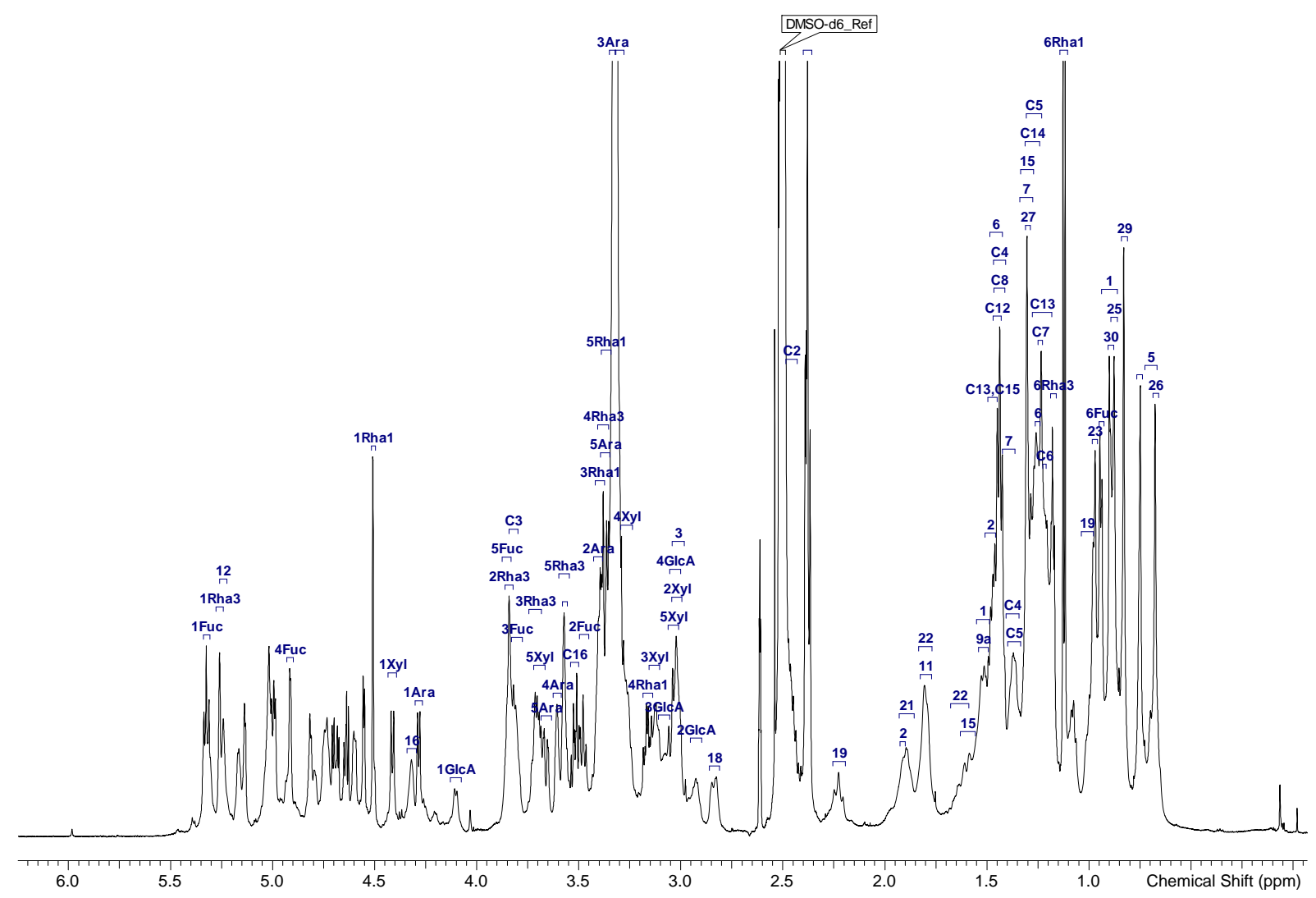

Figure S40. ${ }^{1} \mathrm{H}-\mathrm{NMR}$ spectrum of compound 7 (600 MHz, DMSO-d 6 ).

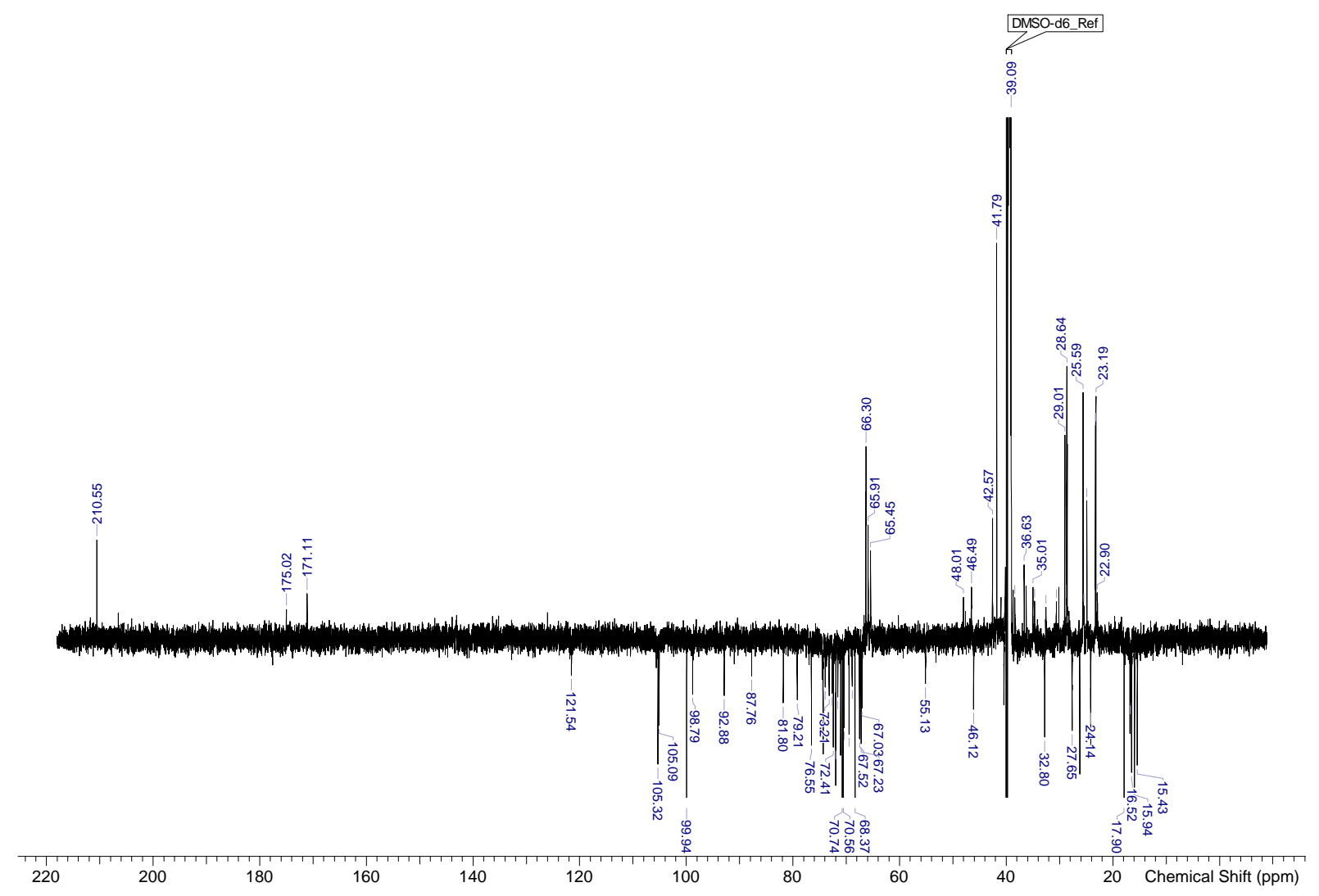

Figure S41. ${ }^{13} \mathrm{C}-\mathrm{DEPTq}$ spectrum of compound 7 (151 MHz, DMSO-d $)$. 


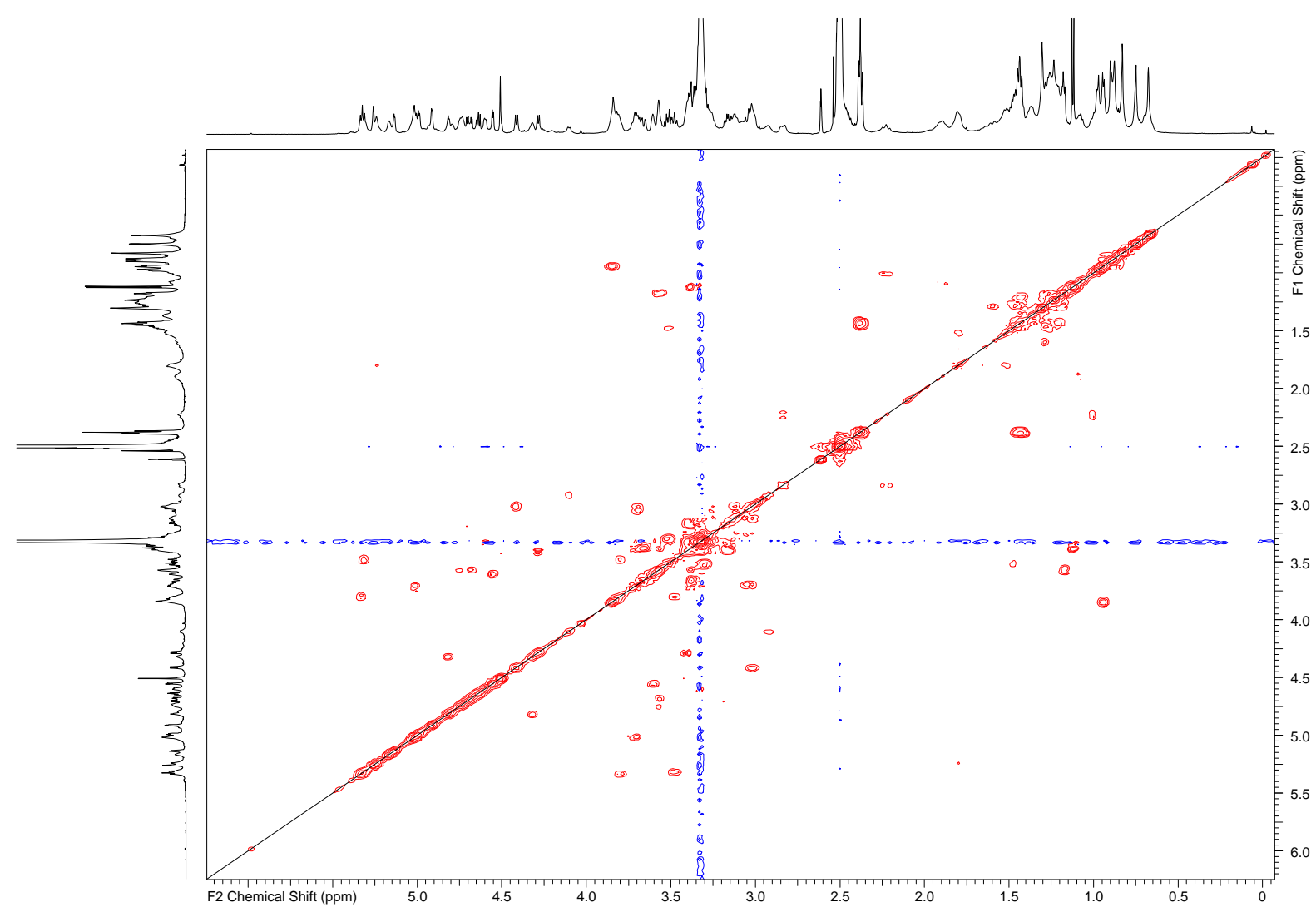

Figure S42. ${ }^{1} \mathrm{H}^{1}{ }^{\mathrm{H}} \mathrm{COSY}$ spectrum of compound 7 (600 MHz, DMSO-d $)$.

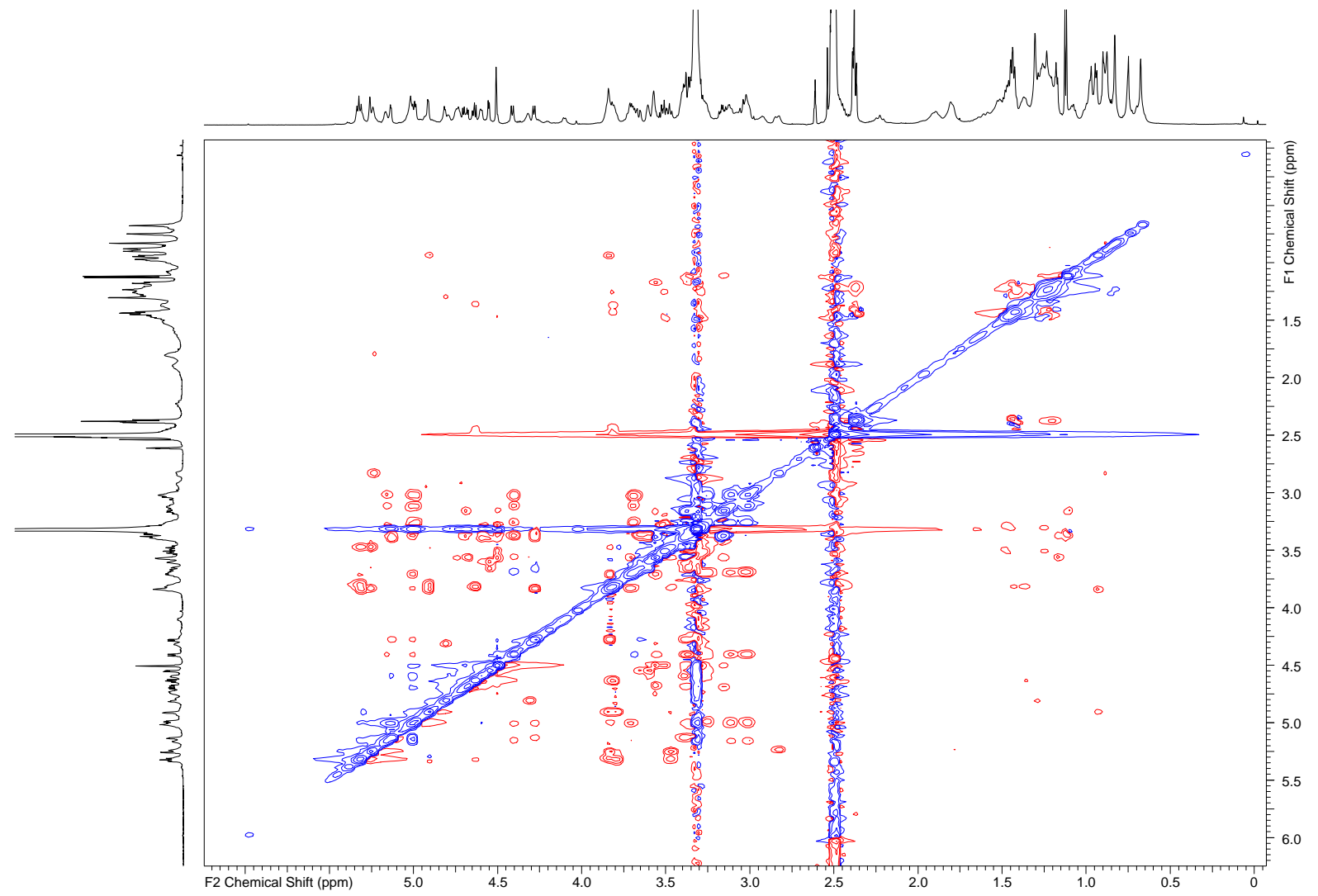

Figure S43. ${ }^{1} \mathrm{H}-{ }^{-1} \mathrm{H}$ ROESY spectrum of compound 7 (600 MHz, DMSO-d6). 


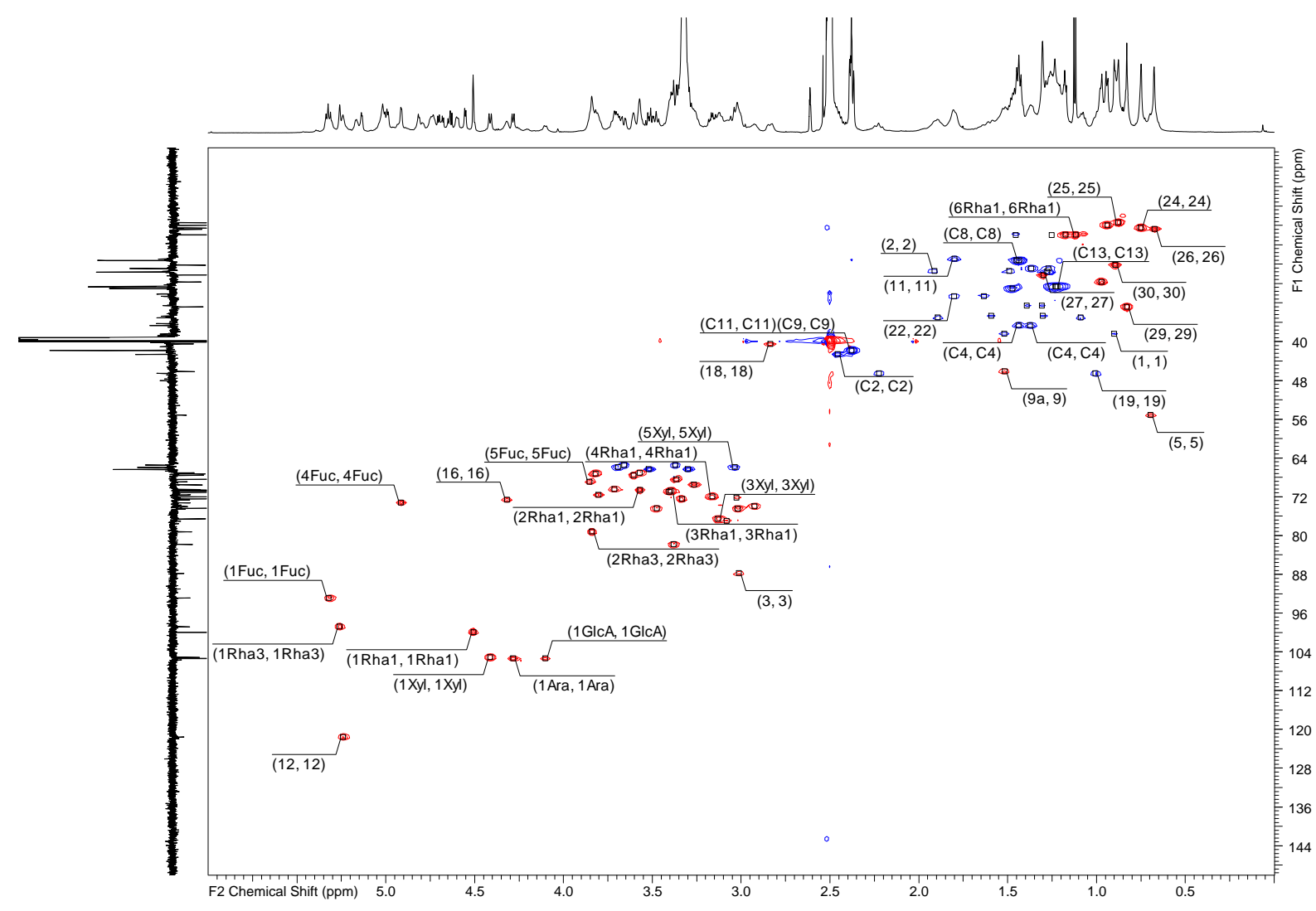

Figure S44. HSQC-DEPT spectrum of compound 7 (600 MHz, DMSO-d6).

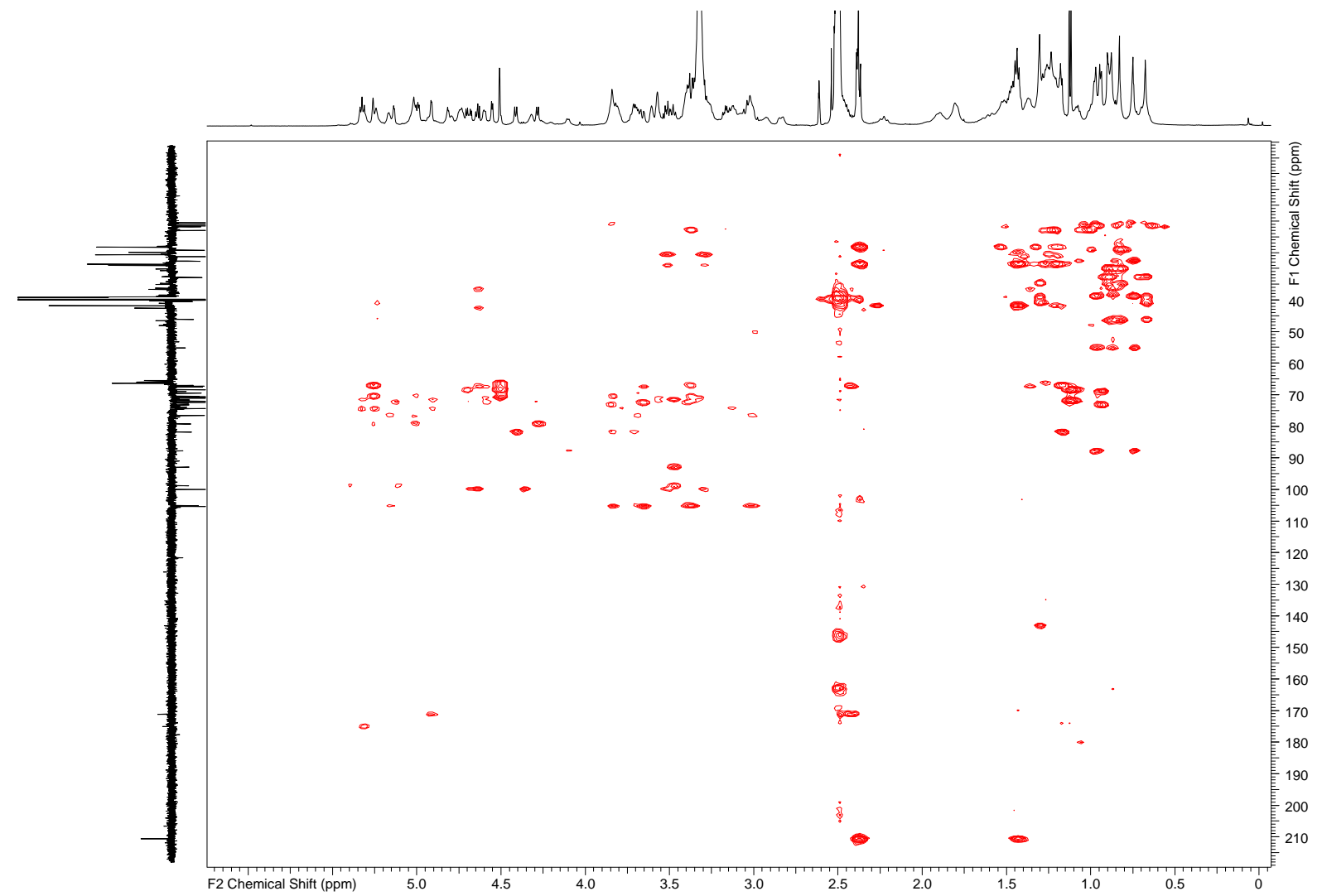

Figure S45. HMBC spectrum of compound 7 (600 MHz, DMSO-d $)$. 


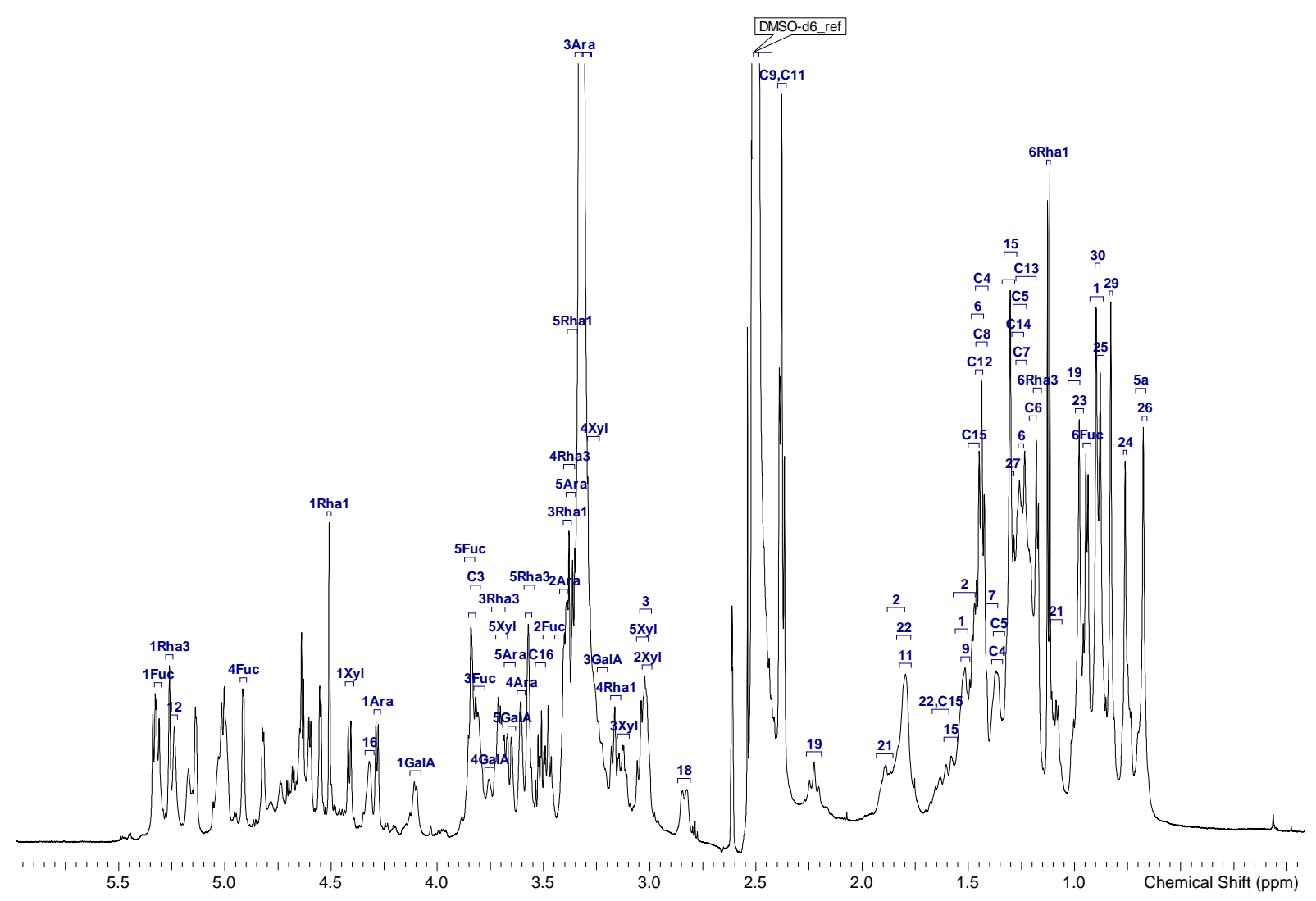

Figure S46. ${ }^{1} \mathrm{H}-\mathrm{NMR}$ spectrum of compound 8 (600 MHz, DMSO-d6).

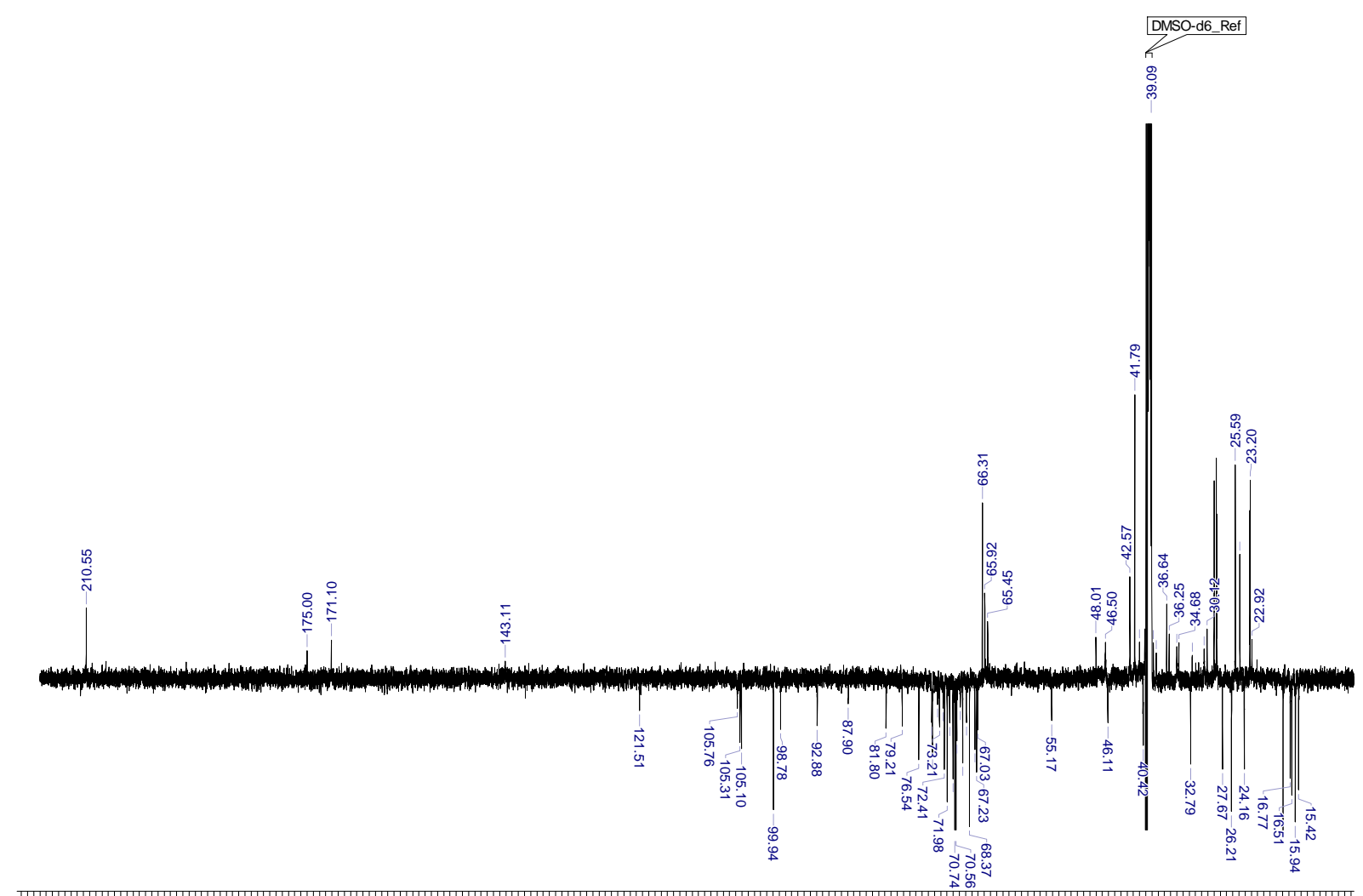

Figure S47. ${ }^{13} \mathrm{C}-\mathrm{DEPTq}$ spectrum of compound 8 (151 MHz, DMSO-d 6 ). 


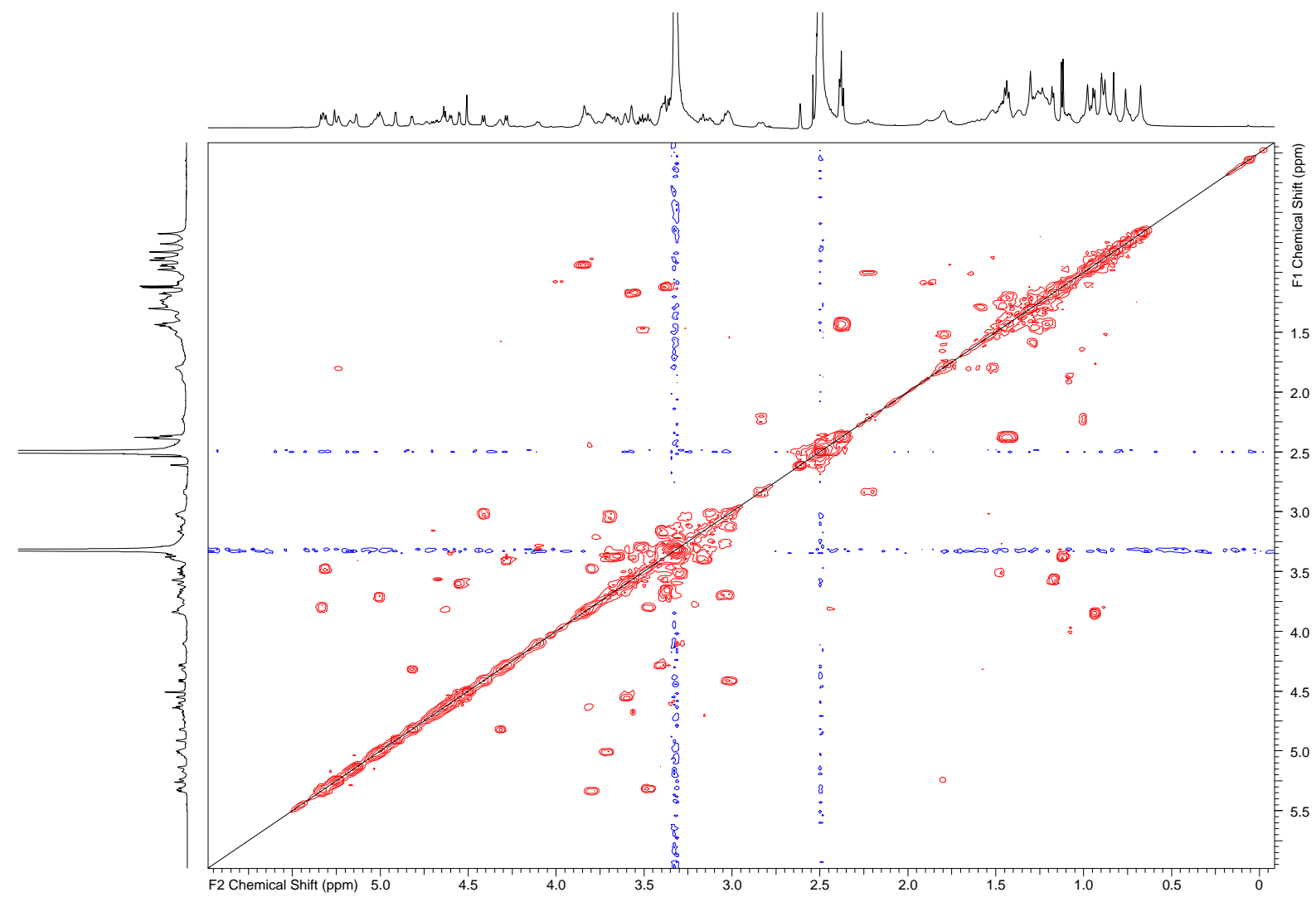

Figure S48. ${ }^{1} \mathrm{H}-{ }^{1} \mathrm{H}$ COSY spectrum of compound 8 (600 MHz, DMSO-d6).

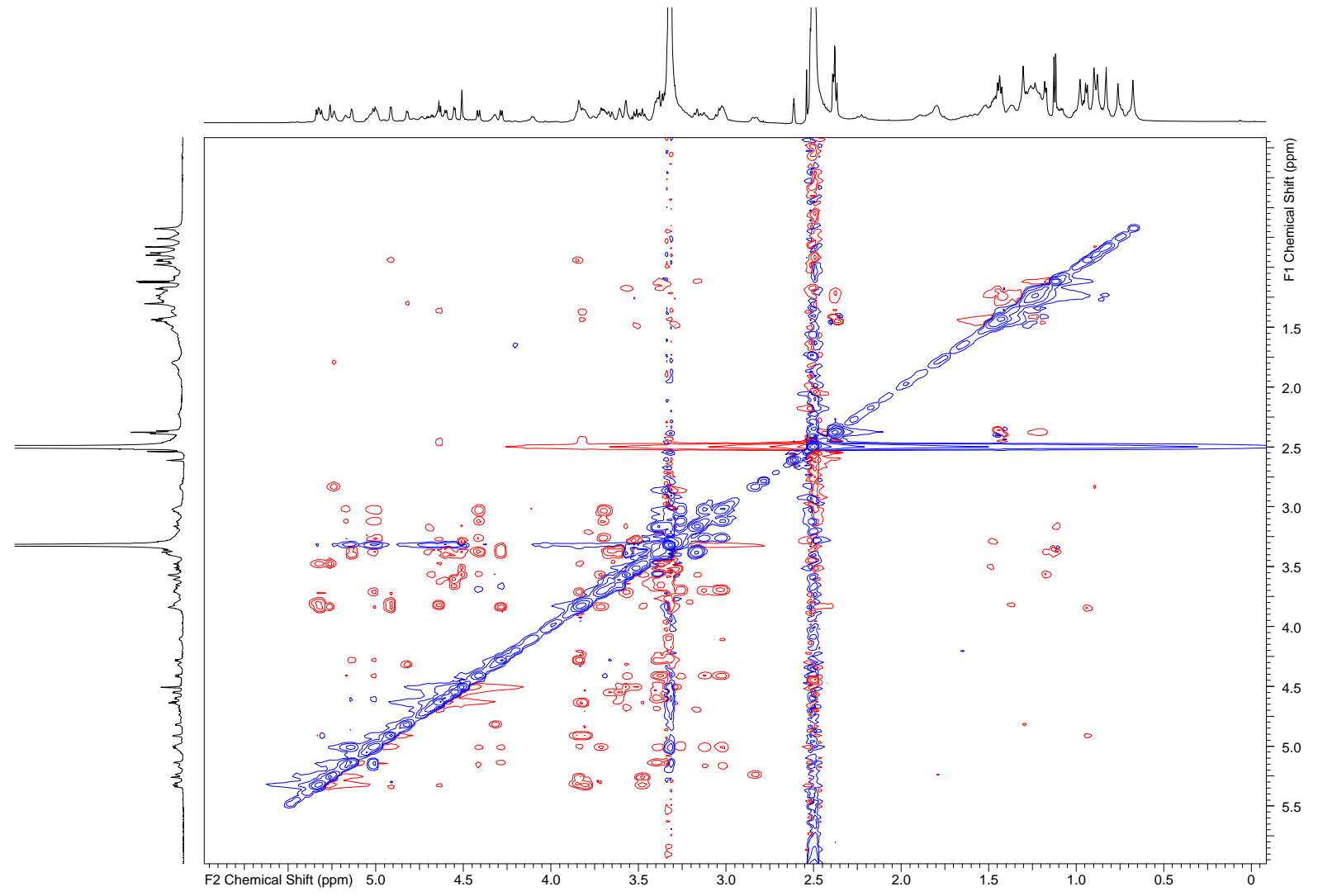

Figure S49. ${ }^{1} \mathrm{H}-{ }^{1} \mathrm{H}$ ROESY spectrum of compound 8 (600 MHz, DMSO-d6). 


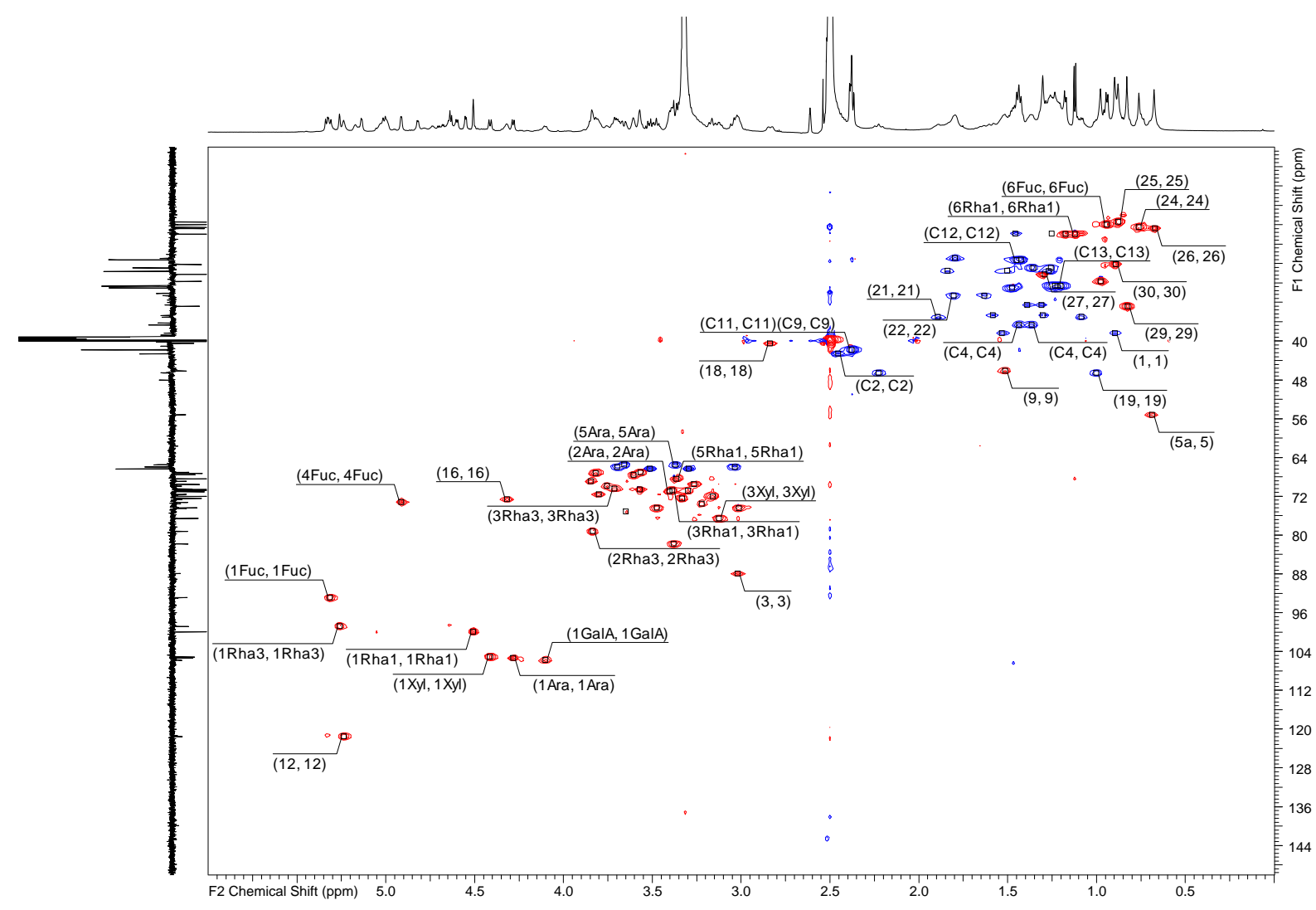

Figure S50. HSQC-DEPT spectrum of compound 8 (600 MHz, DMSO-d6).

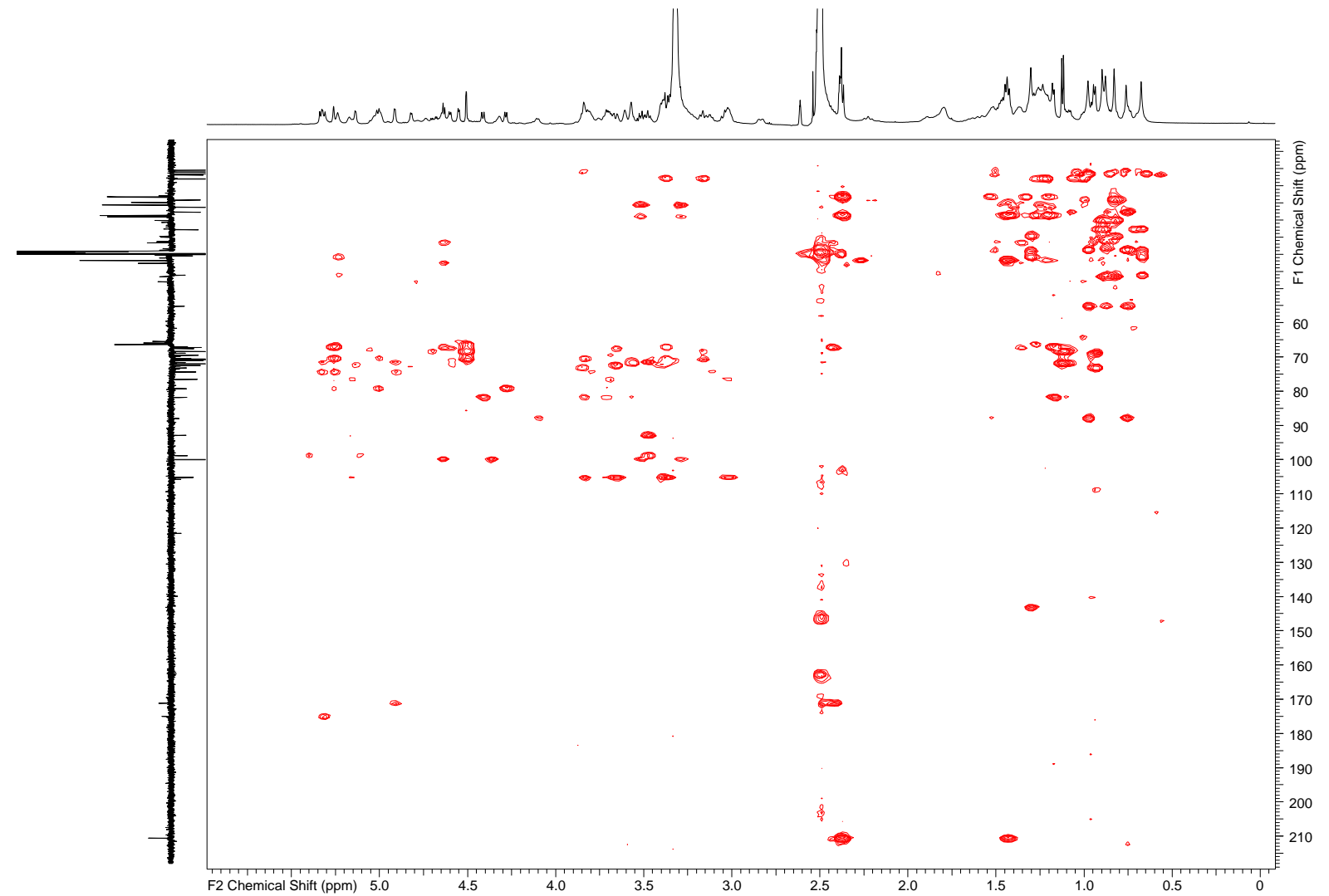

Figure S51. HMBC spectrum of compound 8 (600 MHz, DMSO-d $)$. 


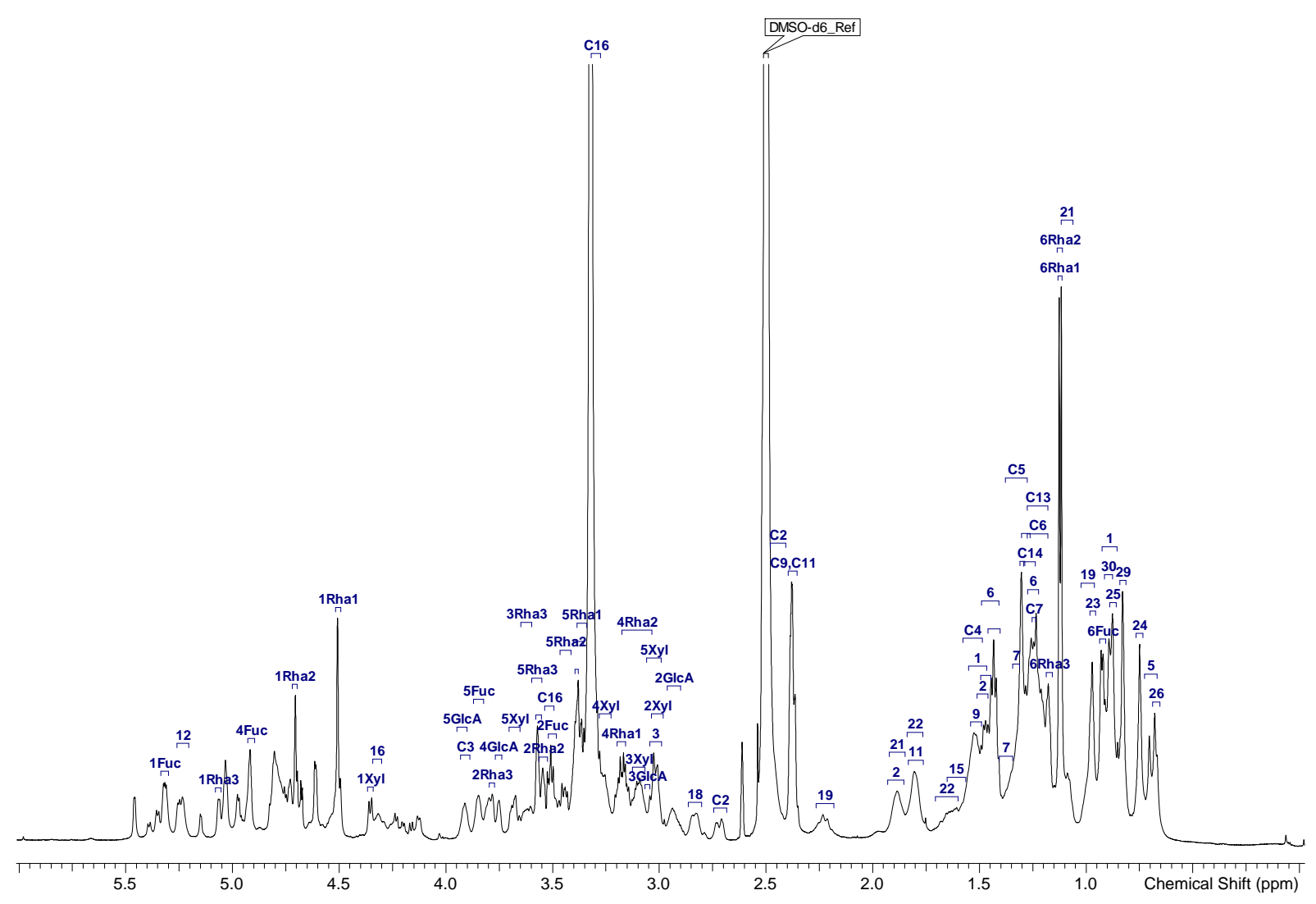

Figure S52. ${ }^{1} \mathrm{H}-\mathrm{NMR}$ spectrum of compound 9 (600 MHz, DMSO-d 6 ).

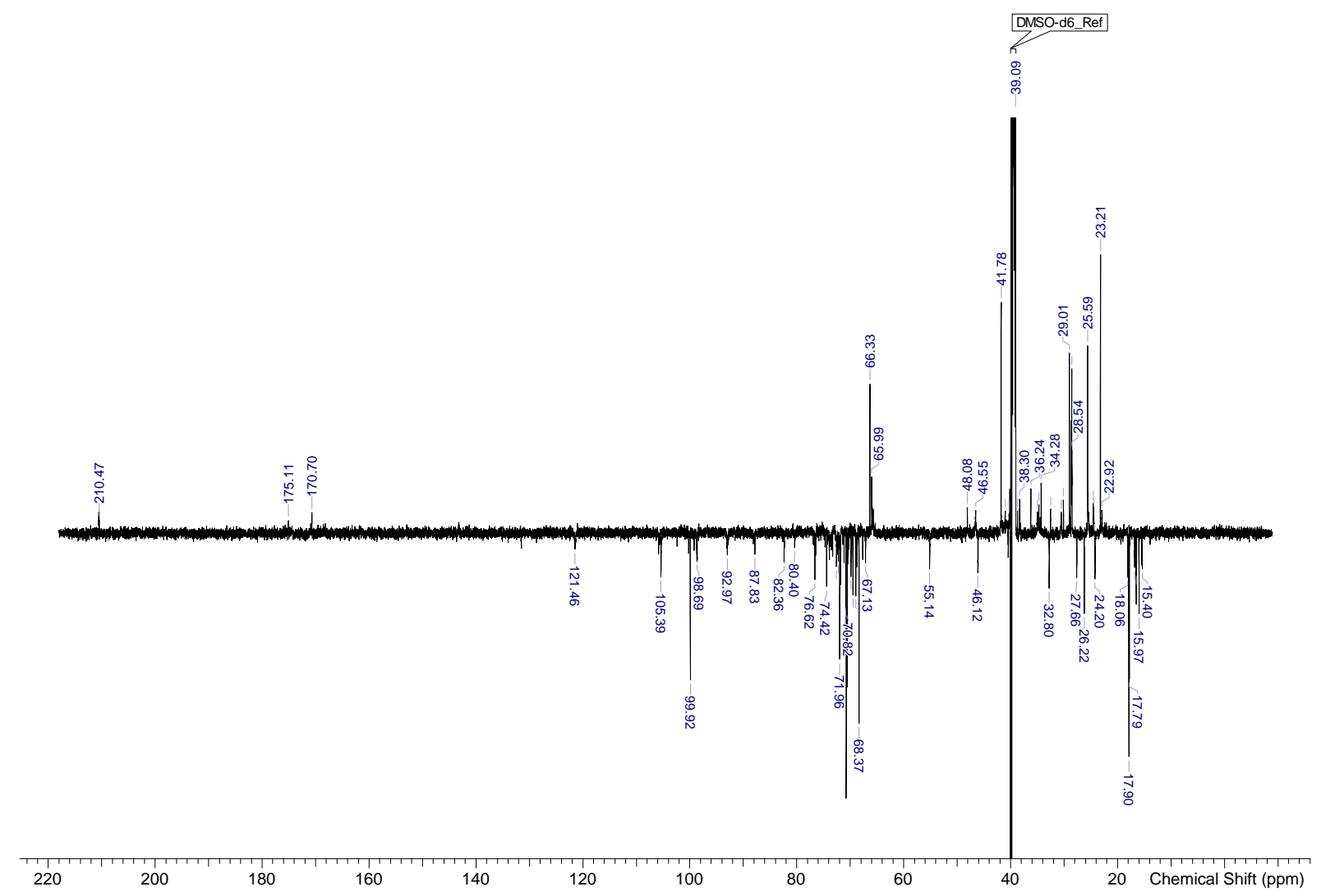

Figure S53. ${ }^{13} \mathrm{C}-\mathrm{DEPTq}$ spectrum of compound 9 (151 MHz, DMSO-d 6 ). 


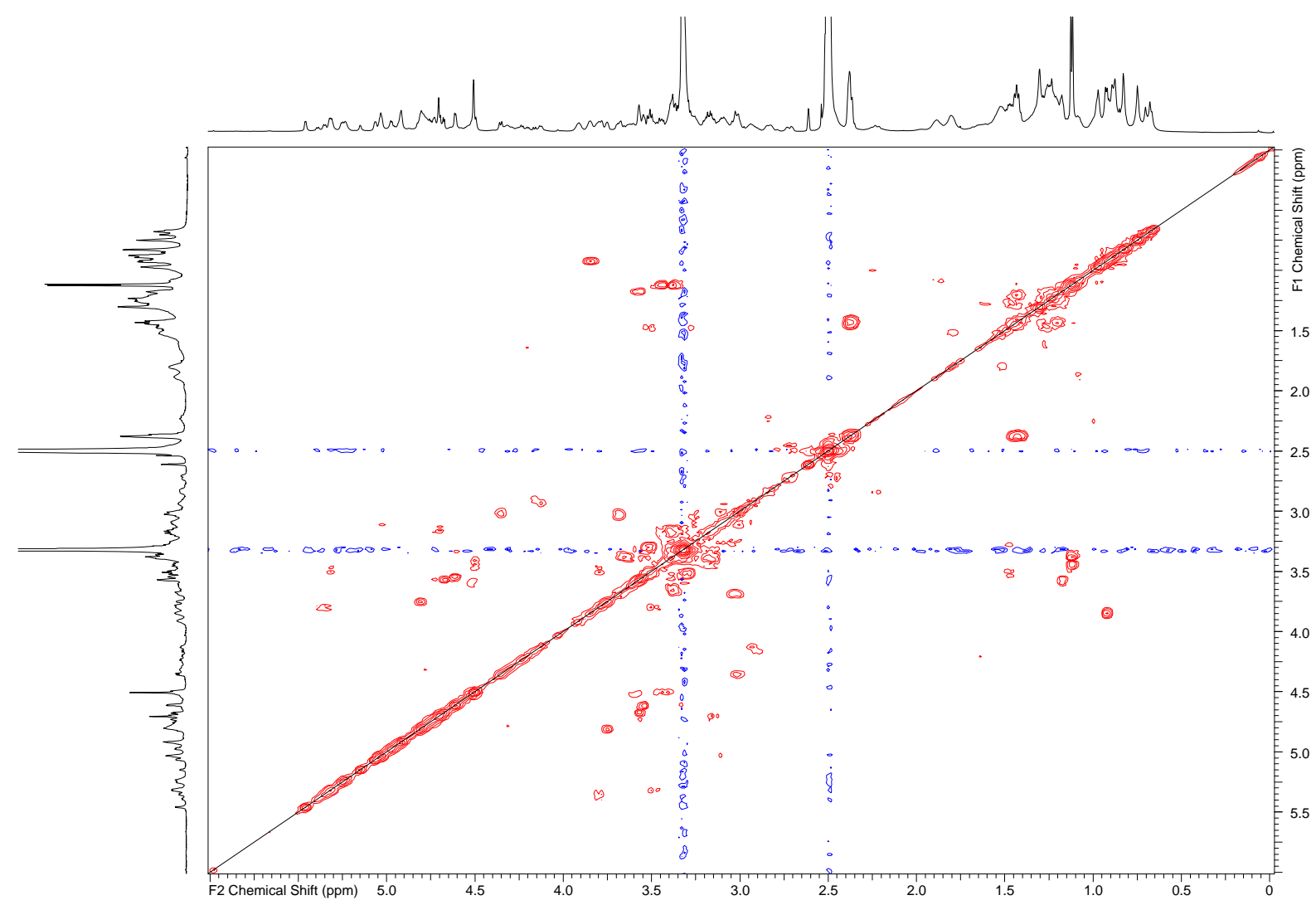

Figure S54. ${ }^{1} \mathrm{H}-{ }^{1} \mathrm{H}$ COSY spectrum of compound 9 (600 MHz, DMSO-d 6 ).

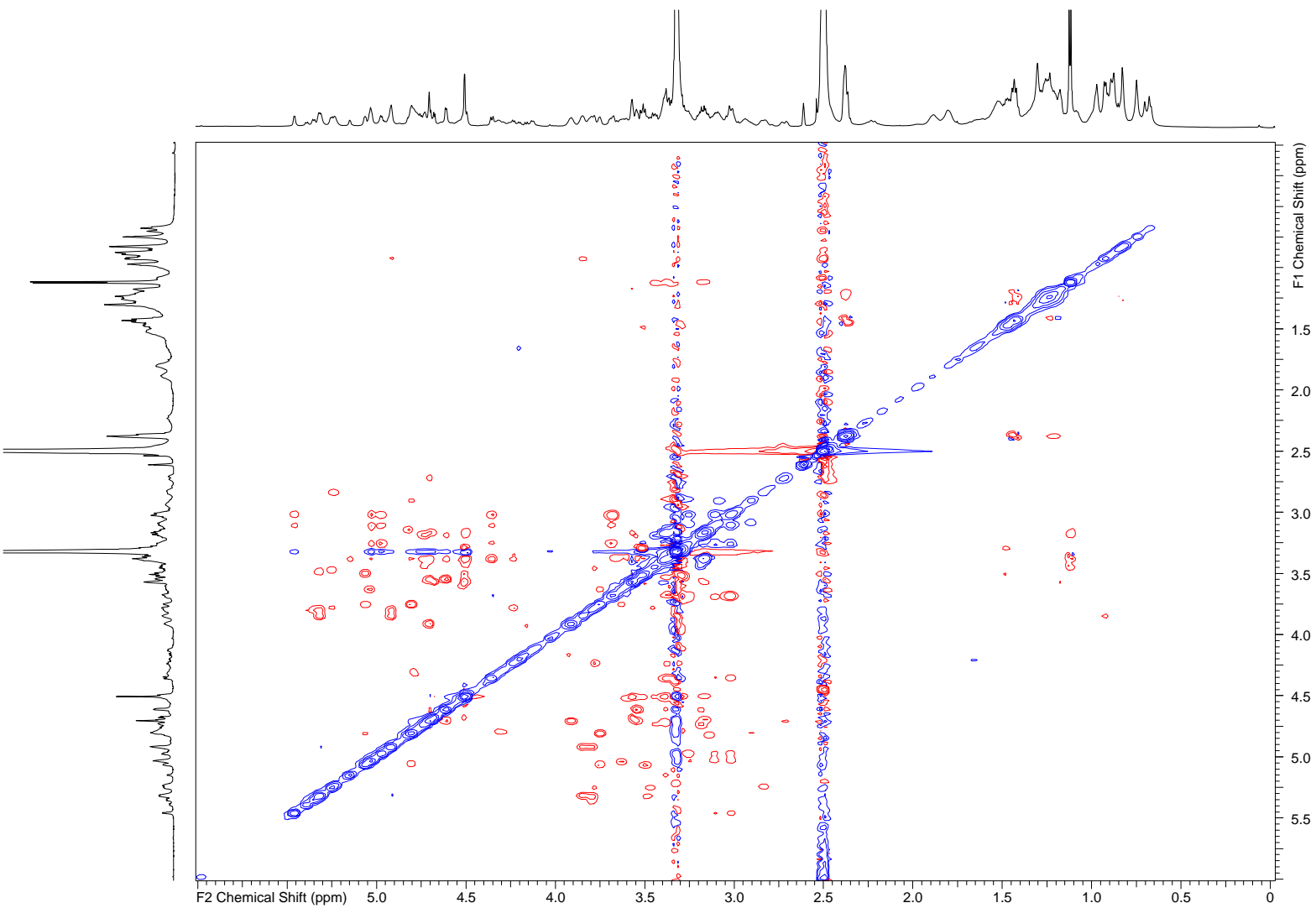

Figure S55. ${ }^{1} \mathrm{H}-{ }^{1} \mathrm{H}$ ROESY spectrum of compound 9 (600 MHz, DMSO-d 6 ). 


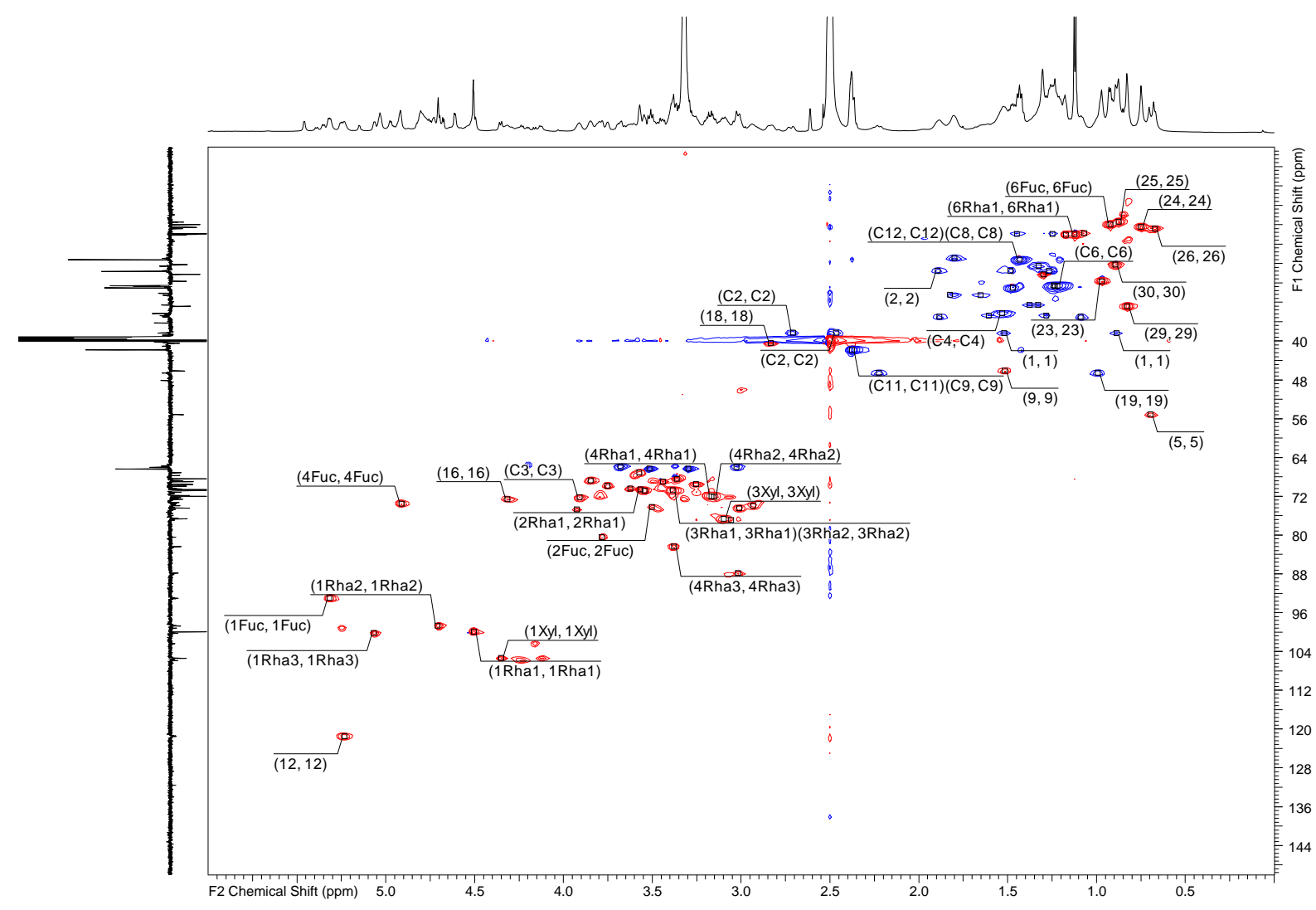

Figure S56. HSQC-DEPT spectrum of compound 9 (600 MHz, DMSO-d6).

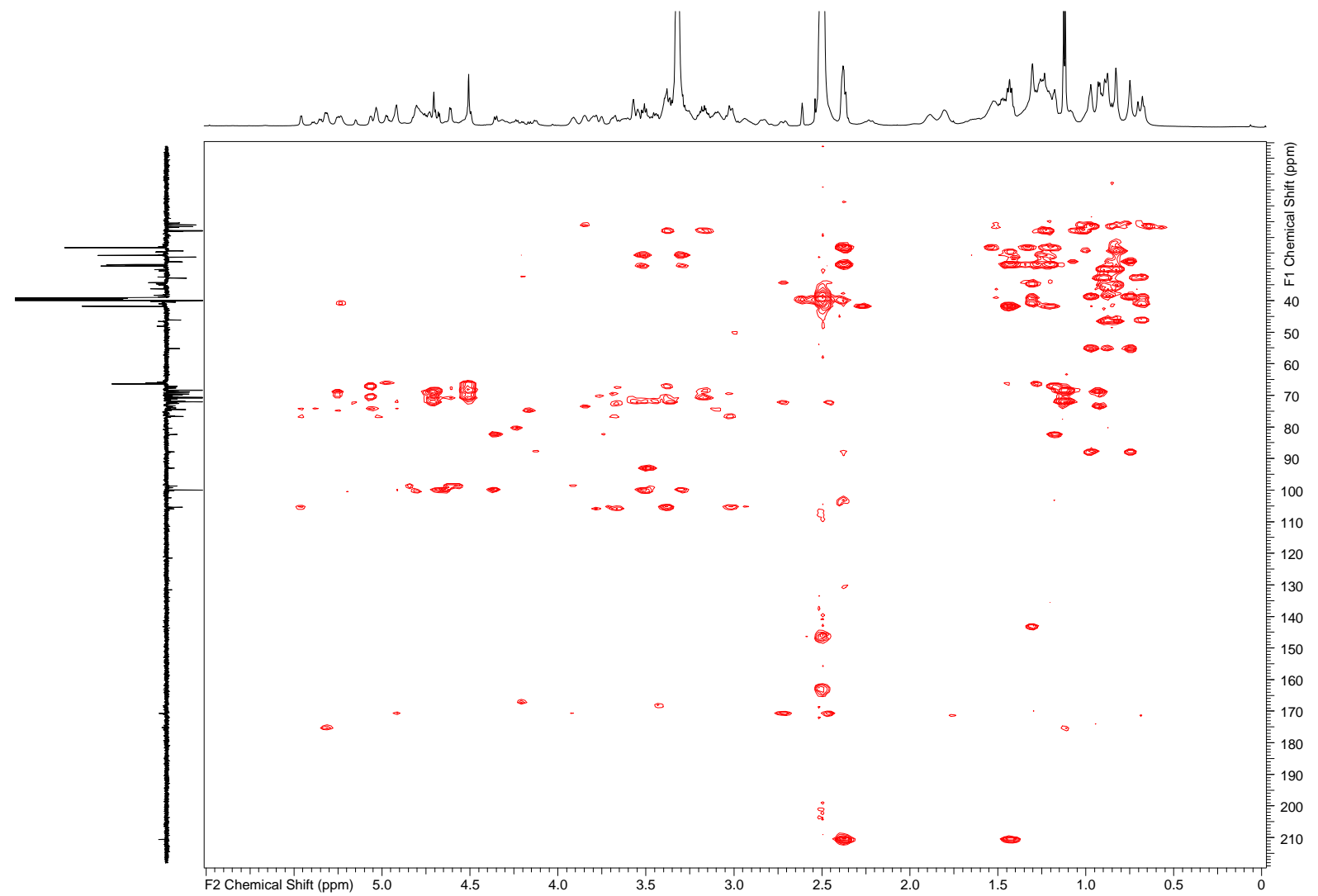

Figure S57. HMBC spectrum of compound 9 (600 MHz, DMSO-d $)$. 


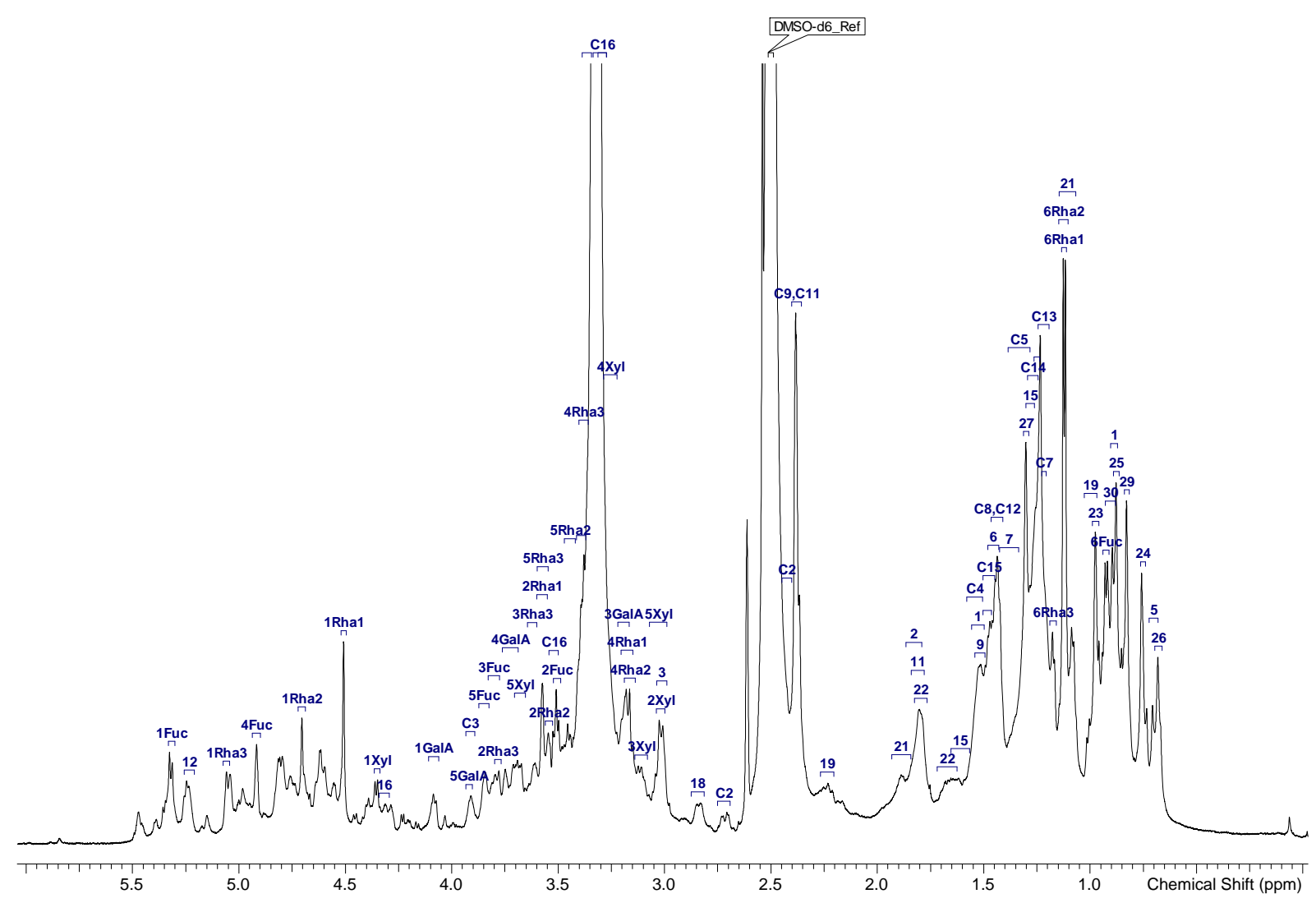

Figure S58. ${ }^{1} \mathrm{H}-\mathrm{NMR}$ spectrum of compound $10\left(600 \mathrm{MHz}, \mathrm{DMSO}-\mathrm{d}_{6}\right)$.

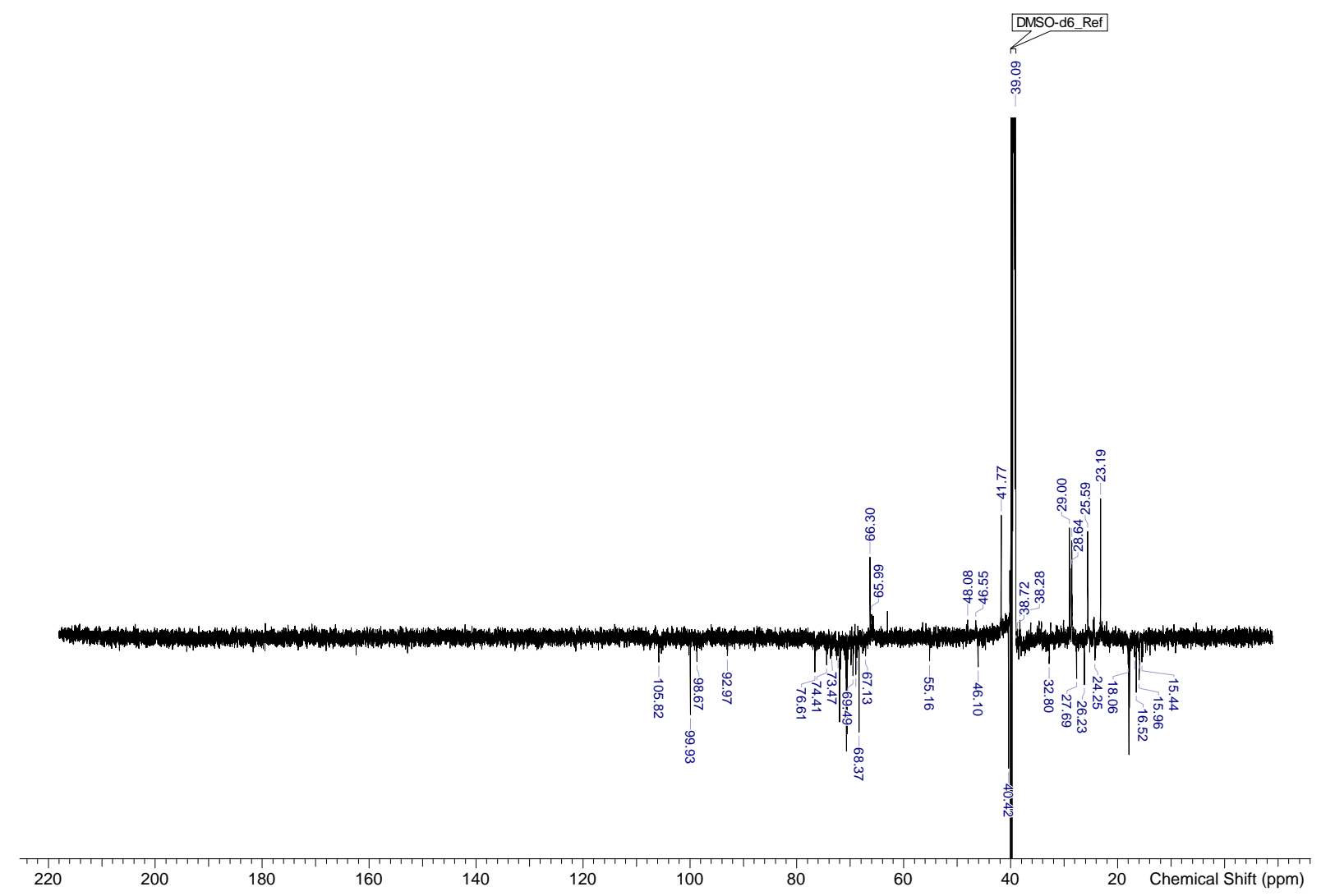

Figure S59. ${ }^{13} \mathrm{C}$-DEPTq spectrum of compound 10 (151 MHz, DMSO-d 6 ). 


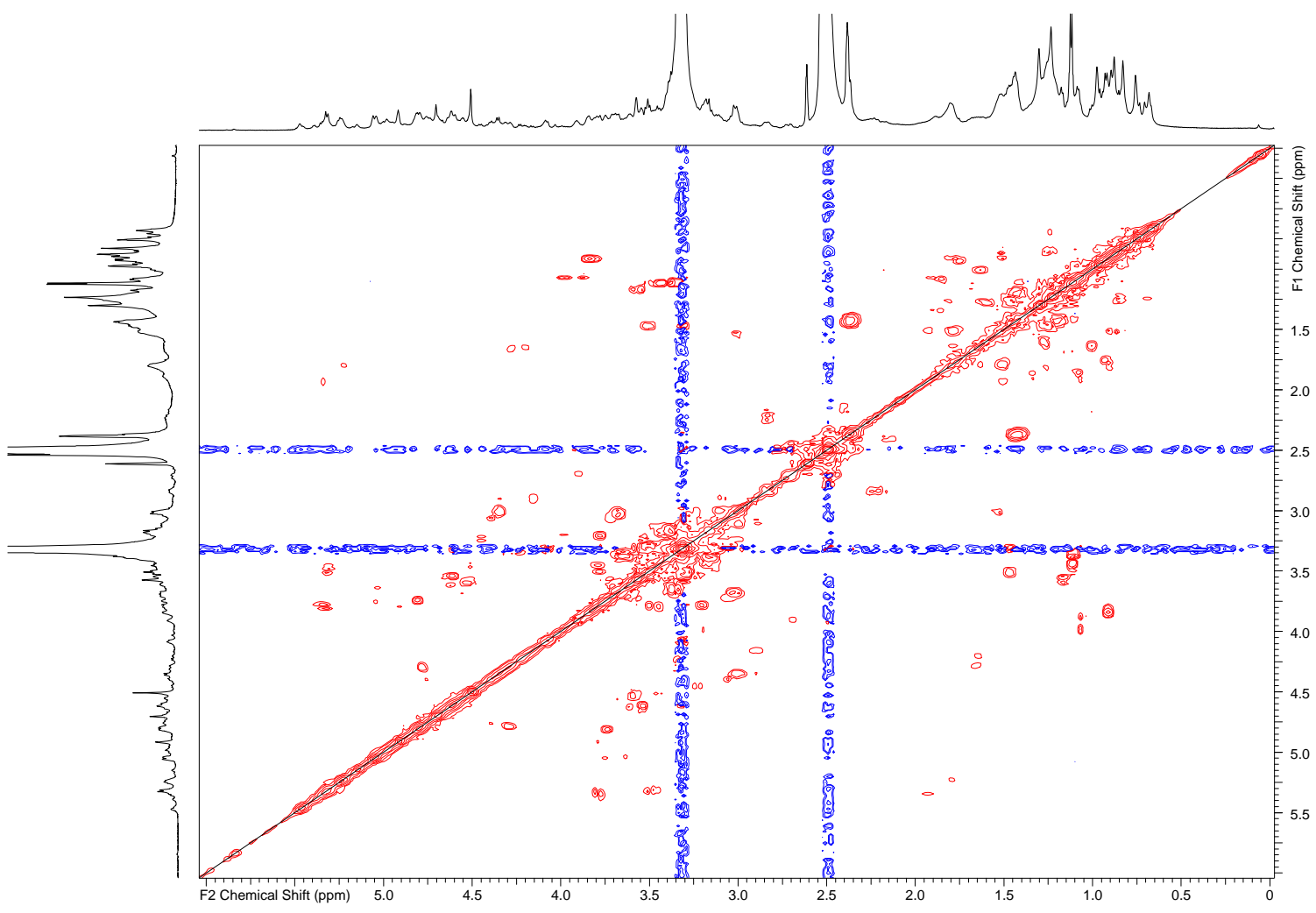

Figure S60. ${ }^{1} \mathrm{H}-{ }^{1} \mathrm{H}$ COSY spectrum of compound $10\left(600 \mathrm{MHz}, \mathrm{DMSO}-\mathrm{d}_{6}\right)$.

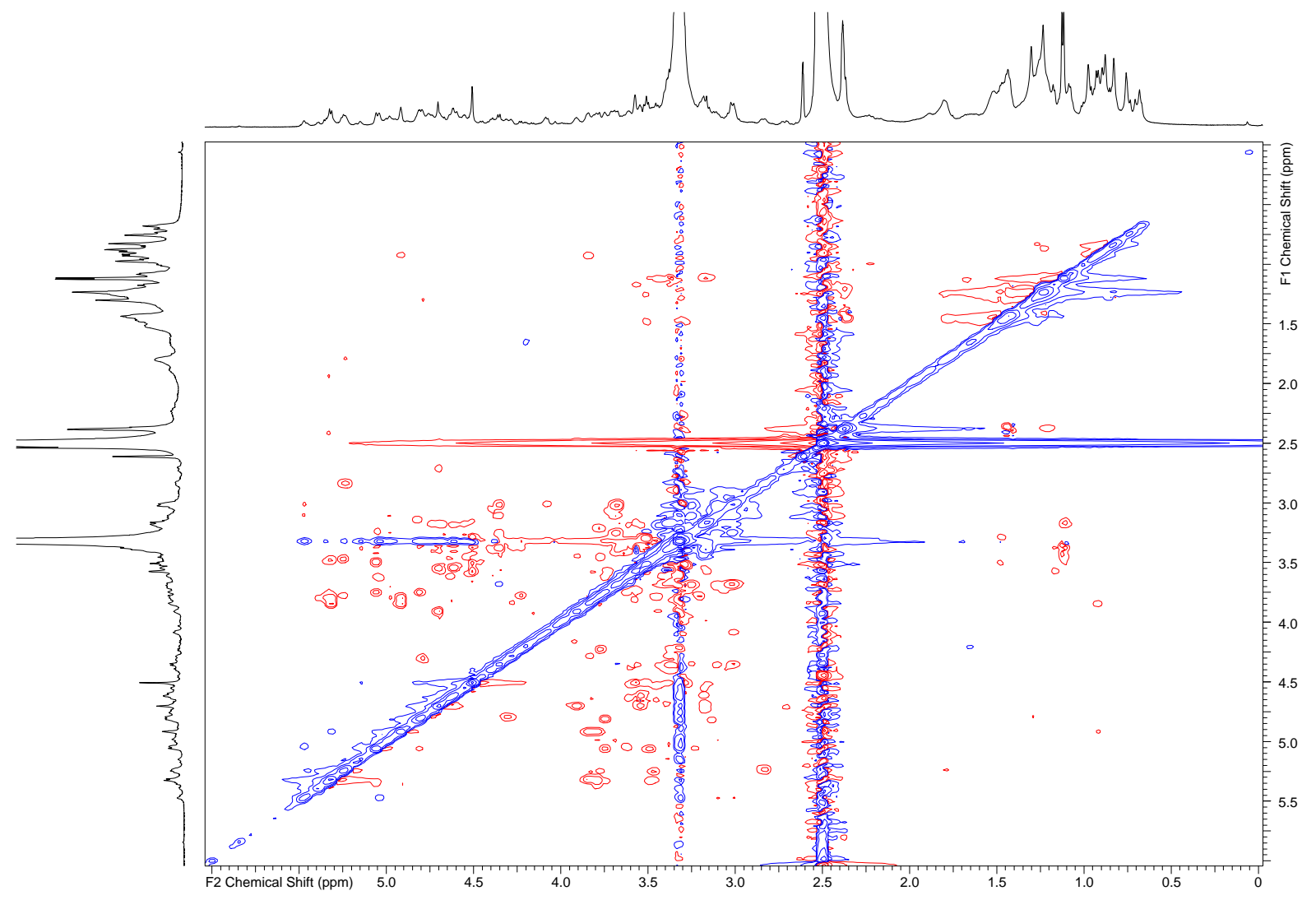

Figure S61. ${ }^{1} \mathrm{H}-{ }^{1} \mathrm{H}$ ROESY spectrum of compound 10 (600 MHz, DMSO-d6). 


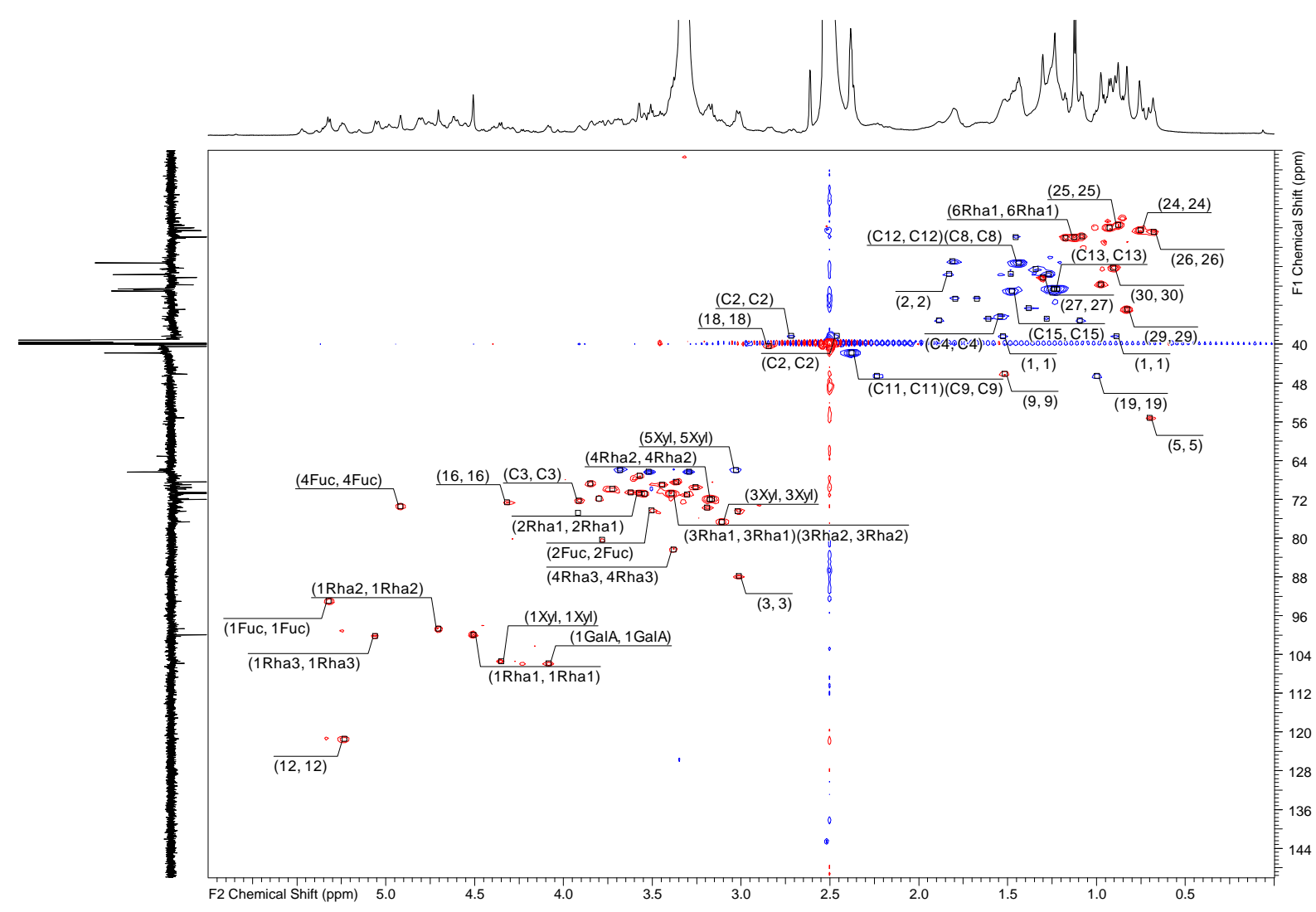

Figure S62. HSQC-DEPT spectrum of compound 10 (600 MHz, DMSO-d6).

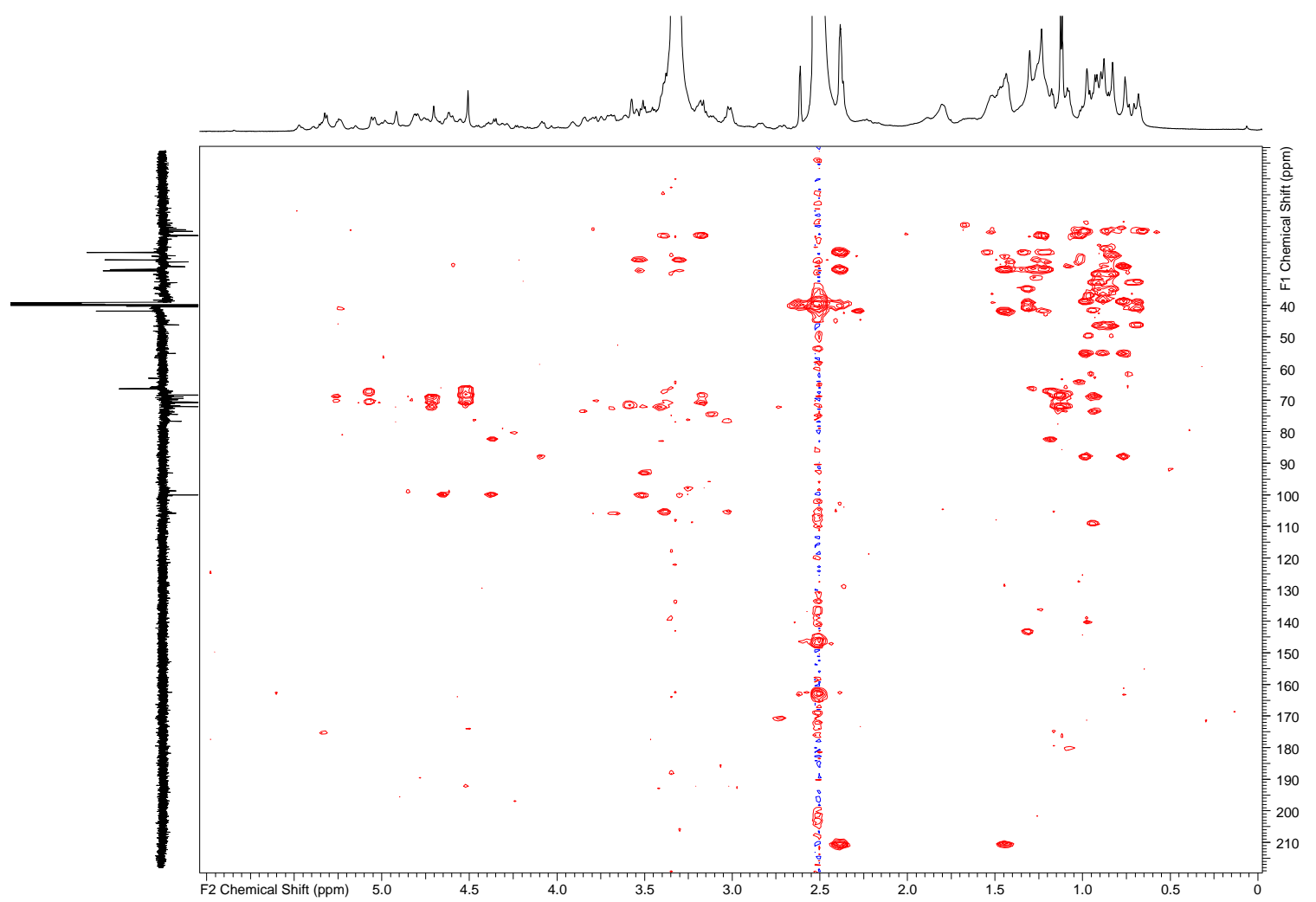

Figure S63. HMBC spectrum of compound 10 (600 MHz, DMSO-d $)$. 